\title{
DuAlity TheOry FOR P-TH POWER FACTORABle Operators and Kernel Operators
}

\author{
Ph. D. DISSERTATION
}

\author{
Presented in the
}

Universitat Politècnica de València

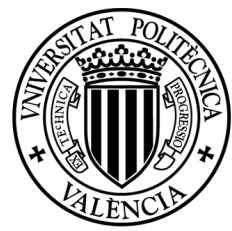

UNIVERSITAT

POLITĖCNICA

DE VALÈNCIA

By

Orlando Eduardo Galdames Bravo

July 2013

Advisors:

Fernando Mayoral Masa

and

Enrique Alfonso Sánchez Pérez 

Don Fernando Mayoral MaSA, profesor del Departamento de Matemática Aplicada II de la Universidad de Sevilla y Don EnRIQUe Alfonso SÁnCHEZ PÉREZ, profesor del Departamento de Matemática Aplicada de la Universidad Politécnica de Valencia,

CERTIFICAN que la presente memoria Duality Theory for p-th Power Factorable Operators and Kernel Operators ha sido realizada bajo su dirección en el Departamento de Matemática Aplicada de la Universidad Politécnica de Valencia, por ORLANDO Eduardo Galdames Bravo y constituye su tesis para optar al grado de Doctor en Ciencias Matemáticas.

Y para que así conste, en cumplimiento de la legislación vigente, presentamos ante el Departamento de Matemática Aplicada de la Universidad Politécnica de Valencia, la referida Tesis Doctoral, firmando el presente certificado.

En Valencia, a 11 de julio de 2013 



\section{AgRADECIMIENTOS}

Quiero agradecer ante todo el apoyo incondicional y el ánimo constante de mis directores de tesis: Enrique y Fernando. Muchas gracias. También a los profesores Luis Miguel García, José Manuel Calabuig, Arantxa Juan, Eduardo Jiménez, Irene Ferrando, Olvido Delgado, Antonio Fernández, Francisco Naranjo, Ricardo del Campo, Pilar Rueda y José Rodríguez. Todos ellos han colaborado de diversas maneras a que este proyecto de tesis viera al fin la luz. También quiero acordarme de mis compañeros de doctorado, Javier Aroza y Javier Falcó, por el estupendo ambiente (de trabajo) que hubo en clase. Y agradecer al profesor José Martínez Alfaro el apoyo que me ofreció en mis inicios cuando estudiaba la topología de sistemas dinámicos, y también por su posterior seguimiento.

No debo olvidar a mi familia. Sin su ayuda esto no habría sido posible. Eva, Gabriel y Marisol: gracias por cuidar de nuestros nenes. Y por supuesto a mis padres: Orlando y Viviana, a los que jamás podré agradecer todo lo que han hecho por mí.

Desde el punto de vista técnico, vuelvo a agradecer a los profesores de la Universidad de Sevilla: Fernando Mayoral, Antonio Fernández, Francisco Naranjo, Ricardo del Campo. Parte de las ideas sobre interpolación que allí trabajamos forman ahora parte de esta tesis. Al profesor Susumu Okada, que con sus explicaciones y sugerencias pude ahondar en la teoría de operadores factorizables a través del espacio de potencias. También, de la Universidad Politécnica de Valencia, a Arantxa Juan, que me orientó con sus conocimiento sobre retículos, José Manuel Calabuig y Luis Miguel García que me ayudaron sobre todo en mis dudas de base, a Eduardo Jiménez por su ayuda sobre la burocracia necesaria para la defensa de una tesis. Y finalmente y de nuevo a mis directores, Fernando Mayoral y Enrique Sánchez, gracias a vosotros ahora puedo escribir esto. 

Ésta se la dedico a mis dos fieras:

Gabriel y Guillem 

Good mathematicians see analogies.

Great mathematicians see analogies between analogies.

Stefan Banach.

Imagination is more important than knowledge.

Albert Einstein. 



\section{RESUMEN}

El presente trabajo está dedicado al análisis de una clase particular de operadores (lineales y continuos) entre espacios de Banach de funciones. El objetivo es avanzar en la teoría de los llamados operadores factorizables a la $p$-potencia analizando todos los aspectos de la dualidad. Esta clase de operadores ha demostrado ser de utilidad tanto en la teoría de factorización de operadores sobre espacios de Banach de funciones teoría de Maurey-Rosenthal) como en el Análisis Armónico (dominios óptimos de la transformada de Fourier y operadores de convolución). A fin de desarrollar esta teoría de dualidad y sus aplicaciones, se define y estudia una nueva clase de operadores con propiedades de extensión que involucran al operador y a su adjunto. Ésta es la familia de operadores factorizables a la $(p, q)$-potencia, $1 \leq p, q<\infty$, y pueden caracterizarse mediante un esquema de factorización a través del espacio de $p$-potencias del dominio y el dual del espacio de $q$-potencias del dual del codominio. También se obtiene una equivalencia mediante un diagrama de factorización a través de espacios $L^{p}(m)$ y $L^{q}(n)^{\prime}$, donde $m$ y $n$ son medidas vectoriales adecuadas y ésta será nuestra principal herramienta (Capítulo 3 y Capítulo 4 ). Para esta construcción resultan necesarios algunos resultados preliminares relativos a las $p$-potencias de los espacios de Banach de funciones que intervienen (Capítulo 2).

Con estos útiles se dan algunos resultados para caracterizar el rango óptimo —el menor espacio de Banach de funciones en el que puede tomar valores el operador- para operadores que van de un espacio de Banach a un espacio de Banach de funciones (Capítulo 3). Además, se desarrolla y presenta formalmente la idea de factorización óptima de un operador que optimiza una factorización previa, en términos del diagrama que debe satisfacer un operador factorizable a su $(p, q)$-potencia (Capítulo 4). Todos estos resultados extienden los actuales cálculos del dominio óptimo mediante medidas vectoriales para operadores sobre espacios de Banach de funciones. Dichos cálculos han dado resultados relevantes en diversas áreas del análisis matemático mediante una descripción del mayor espacio de Banach de funciones al cual, operadores relevantes - como la transformada de Fourier o el operador de Hardy_ se pueden extender.

La teoría se aplica para encontrar nuevos resultados en determinados campos: como la teoría de interpolación de operadores entre espacios de Banach de funciones, los operadores de núcleo (Capítulo 5) y en particular, la transformada de Laplace (Capítulo 6). 



\section{RESUM}

El present treball es dedica a l'anàlisi d'una classe concreta d'operadors (lineals i continus) entre espais de Banach de funcions. L'objectiu és avançar en la teoria dels anomenats operadors factoritzables a la $p$-potència analitzant tots els aspectes de la dualitat. Aquesta classe d'operadors ha demostrat ser útil tant a la teoria de factorització d'operadors sobre espais de Banach de funcions (teoria de Maurey-Rosenthal) com en l'Anàlisi Harmònic (dominis òptims de la transformada de Fourier i d'operadors de convolució). Per tal de desenvolupar la corresponent teoria de dualitat i les aplicacions, es defineixen i estudien una nova classe d'operadors amb propietats d'extensió que involucren a l'operador $\mathrm{i}$ al seu adjunt. Aquesta és la familia d'operadors factoritzables a la $(p, q)$-potència, $1 \leq$ $p, q<\infty$, els quals es poden caracteritzar mitjançant un diagrama de factorització per l'espai de $p$-potències del domini i el dual de l'espai de $p$-potències del dual del codomini. També s'obté una equivalència per mig d'un diagrama de factorització a través de $L^{p}(m)$ i $L^{q}(n)^{\prime}$ per a unes mesures vectorials $m$ i $n$ convenients, i que serà la nostra principal eina (Capítol 3 i Capítol 4). Per dur a terme aquesta tasca es necessiten alguns resultats preliminars en relació amb els espais de $p$-potència dels espais de Banach de funcions que actuen (Capítol 2).

Amb estes eines es dónen alguns resultats per caracteritzar el rang òptim —el menor espai de Banach de funcions al que pot prendre valors l'operador- per a operadors que van des d'un espai de Banach fins un espai de Banach de funcions (Capítol 3). A més a més, es presenta i desenvolupa la idea de factorització òptima d'un operador que optimitza una factorització prèvia en termes del diagrama de factorizació que satisfà un operador factoritzable a la $(p, q)$-potència (Capítol 4$)$. Tots aquests resultats extenen els actuals càlculs del domini òptim mitjançant mesures vectorials per a operadors sobre espais de Banach de funcions. Aquests càlculs han donat resultats rellevants en diverses àrees de l'anàlisi matemàtic mitjançant una descripció del major espai de Banach de funcions al qual, operadors relevants — com la transformada de Fourier o l'operador de Hardy— es poden extendre.

La teoria s'aplica per trobar nous resultats en alguns camps concrets: como ara la teoria d'interpolació d'operadors entre espais de Banach de funcions, els operadors de nucli (Capítol 5) i en particular, la transformada de Laplace (Capítol 6). 



\begin{abstract}
The present work is devoted to the analysis of a particular class of (linear and continuous) operators between Banach function spaces. The aim is to advance in the theory of the so-called $p$-th power factorable operators by analyzing all the aspects of the duality. This class of operators has proved to be useful both in the factorization theory of operators on Banach function spaces (Maurey-Rosenthal theory) and in Harmonic Analysis (optimal domains for the Fourier transform and convolution operators). In order to develop the corresponding duality theory and some applications, a new class of operators with extension properties involving both the operator and its adjoint is defined and studied. This is the family of the $(p, q)$-th power factorable operators, for $1 \leq p, q<\infty$, that can be characterized by means of a canonical factorization scheme through the $p$-th power space of the domain space and the dual of the $q$-th power space of the dual of the codomain space. An equivalent diagram factoring such an operator through $L^{p}(m)$ and $L^{q}(n)^{\prime}$ for suitable vector measures $m$ and $n$ is also obtained, and this becomes the main tool (Chapter 3 and Chapter 4). Some other preliminary results concerning $p$-th powers of Banach function spaces are also necessary for constructing the above mentioned ones (Chapter 2).

Using these tools, some results characterizing the optimal range -the smallest Banach function space in which the operator can take values- for operators from a Banach space into a Banach function space are given (Chapter 3). Also, the idea of optimal factorization of an operator optimizing a previous one is developed and formally presented in terms of the diagram that a $(p, q)$-th power factorable operator must satisfy (Chapter 4 ). All these results extend the nowadays well known computation of the optimal domain for operators on Banach function spaces by means of vector measures. These computations have provided relevant results in several fields of the mathematical analysis by means of a description of the biggest Banach function spaces to which some special relevant operators - for instance, the Fourier transform and the Hardy operator — can be extended.

The theory is applied for finding new results in some concrete fields: as interpolation theory for operators between Banach function spaces, kernel operators (Chapter 5) and in particular, to the Laplace transform (Chapter 6).
\end{abstract}





\section{CONTENTS}

RESUMEN

RESUM

ABSTRACT

INTRODUCTION

Chapter 1. PRELIminaries AND NOTATION

1.1. Measures and spaces 1

1.1.1. Vector measures 1

1.1.2. Banach lattices and Banach function spaces 2

1.1.3. $L^{p}(m)$ spaces and optimal domains $\quad 4$

1.1.4. The $p$-th power space $\quad 5$

1.2. Operators $\quad 8$

1.2.1. The $p$-th power factorable operators 8

1.2.2. Kernel operators 11

Chapter 2. GENERALIZED KÖthe DUAL SPACE $\quad 13$

2.1. The Köthe $p$-dual space $\quad 13$

2.1.1. Computation rules for the Köthe $p$-dual space $\quad 14$

2.1.2. The Köthe $p$-adjoint operator $\quad 16$

2.2. Some geometrical aspects $\quad 18$

2.2.1. Convexity and concavity $\quad 19$

2.2.2. Type and cotype 21

Chapter 3. OPtimal RANGE THEOREM $\quad 25$

3.1. The adjoint and Köthe adjoint operators 25

3.2. Optimal range theorem $\quad 27$

3.2.1. Applications of the optimal range theorem 31

3.2.2. The optimal range for the Volterra operator 34

Chapter 4. $(p, q)$-TH POWER FACTORABLE OPERATORS 37

4.1. The $(p, q)$-th power factorable operator $\quad 37$

4.1.1. Characterizations for $(p, q)$-th power factorable operators 38

4.1.2. Another related result $\quad 42$ 
4.2. The non finite measure case

Chapter 5. APPLICATIONS OF $(p, q)$-TH POWER FACTORABLE OPERATORS 47

5.1. Complex interpolation for operators $\quad 47$

5.1.1. Definitions and notation $\quad 47$

5.1.2. Interpolation for $p$-th power factorable operators $\quad 49$

5.1.3. Interpolation for $(p, q)$-th power factorable operators $\quad 54$

5.2. $(p, q)$-th power factorable kernel operators $\quad 57$

5.2.1. Hölder's type inequality 58

5.2.2. Hille-Tamarkin operators 60

Chapter 6. AN OPTIMAL DOMAIN FOR THE LAPLACE TRANSFORM 63

6.1. Introduction 63

6.2. The vector measure 64

6.2.1. An embedding property 66

6.2.2. An estimate for the norm 67

6.3. Factorization and optimal domain $\quad 70$

6.3.1. The $(p, q)$-th power factorization $\quad 70$

$\begin{array}{lll}\text { 6.3.2. } & \text { Final remarks and example } & 73\end{array}$

$\begin{array}{ll}\text { BIBLIOGRAPHY } & 77\end{array}$

LIST OF DIAGRAMS

$\begin{array}{ll}\text { INDEX } & 85\end{array}$ 


\section{INTRODUCTION}

The aim of the present work is to provide a duality theory for the so called $p$-th power factorable operators, that have been recently defined and used for the general study of the optimal domain of operators between Banach function spaces and Banach spaces, finding applications in Functional and Fourier Analysis. The idea is to define and show the applications of the class of $(p, q)$-th power factorable operators between Banach function spaces, that are defined in a way that concerns the $p$-th power factorability of the original operator and the $q$-th power factorability of its dual map. We will find applications to some classical topics of the operator theory and spaces of integrable functions, such as interpolation theory and kernel operators, bringing new light to the problem of finding the optimal domain and the optimal range of some classical operators as the Laplace transform.

Let us introduce some basic definitions. Given a Banach function space $X(\mu)$ over a finite measure $\mu$-i.e. a Banach ideal of classes of measurable functions in $L^{0}(\mu)$ - and $p>0$, we can define its $p$-th power space (also called $1 / p$-convexification) as the quasiBanach function space of $\mu$-measurable functions such that the $1 / p$ power of its modulus belongs to $X(\mu)$ itself. This space was explicitly introduced in [42, Defn. 1.9] and [23, p. 156] (see also [71]), and fruitfully applied in [79, Ch. 5] and $[\mathbf{1 7}, \mathbf{2 8}, \mathbf{2 9}]$ to the theory of Optimal Domains, to find representation theorems for Banach lattices in $[\mathbf{1 5}, \mathbf{2 7}, \mathbf{3 8}, \mathbf{5 3}]$ and for extending the Maurey-Rosenthal Theorems and its applications in Fourier Analysis in [79, Ch. 6,7].

Spaces of integrable functions with respect to a vector measure constitute a fundamental tool in our work. In fact, there is a direct association between vector measures and operators, and also between spaces of integrable functions with respect to a vector measure and order continuous Banach functions spaces over a finite measure. Given a Banach space valued operator $T$ from such a space $X(\mu)$ over the finite measure space $(\Omega, \Sigma, \mu)$, the expression $m_{T}(A):=T\left(\chi_{A}\right), A \in \Sigma$, defines a vector measure under some mild conditions on $T$ and $X(\mu)$.

The class of $p$-th power factorable operators was developed in 2008 by Okada, Ricker and Sánchez Pérez in [79, Ch. 5]. This is the family of Banach space valued (linear and continuous) operators from a Banach function space $X(\mu)$ over a finite measure $\mu$ that can be extended to the $p$-th power space $X(\mu)_{[p]}$ on $X(\mu)$. More precisely, if $E$ is a Banach space, 
$1<p<\infty$ and $T: X(\mu) \rightarrow E$ is an operator, there is a continuous operator $T_{[p]}: X(\mu)_{[p]} \rightarrow E$ such that $T=T_{[p]} \circ i_{[p]}$, where $i_{[p]}$ is the natural inclusion map $i_{[p]}: X(\mu) \hookrightarrow X(\mu)_{[p]}$. This is equivalent to the optimality of the extension of the map through the space of $p$-integrable functions with respect to $m_{T}$, the associated vector measure with $T$.

This main idea extends the basic fact that is known in this setting for continuous operators: the optimal domain for $T: X(\mu) \rightarrow E-$ i.e. the maximal space to which the operator can be extended-is the space of $m_{T}$-integrable functions $L^{1}\left(m_{T}\right)$. If we consider an operator that can be extended to $X(\mu)_{[p]}$ for $1<p<\infty$, then its optimal domain preserving the same property, is $L^{p}\left(m_{T}\right)$. This provides direct information about both the operator and the corresponding space of integrable functions $L^{p}\left(m_{T}\right)$ (see [79, Th. 5.7]). More relevant properties of this class of operators is that they are always weakly compact, and $p$-convex when the operator is positive, since $L^{p}\left(m_{T}\right)$ is $p$-convex (see e.g. [66, Th. 1.d.9]).

Duality applied to factorization diagrams for operators is a well known technique for analyzing operators between Banach spaces and Banach lattices (see for instance $[\mathbf{2 3 , 2 4}$, $\mathbf{8 7}, \mathbf{8 8}]$ ). Regarding $p$-th power factorable operators, this is the main motivation of this work. Given a $p$-th power factorable operator $T: X(\mu) \rightarrow E$, there is no reason to think that its dual map is also $p$-th power factorable. However, to know if there is any factorability property for the dual map opens the door to a more detailed analysis of the properties of the operator. This leads us to the definition of a more general class of operators that we will call $(p, q)$-th power factorable operators and are defined as those operators between Banach function spaces $X(\mu)$ and $Y(v)$ which factor as $T=S \circ R$ through a Banach space $E$ and satisfy that $R: X(\mu) \rightarrow E$ is $p$-th power factorable and $S^{\prime}: Y(v)^{\prime} \rightarrow E^{*}$ is $q$-th power factorable, where $S^{\prime}$ is the restriction of the adjoint operator $T^{*}: Y(v)^{*} \rightarrow E^{*}$ to the Köthe adjoint $Y(v)^{\prime}$ of $Y(v)$. The definition of this class is natural and in a sense similar to the Kwapień decomposition for $(p, q)$-factorable operators and to the definition of $(p, q)$ dominated operators due to Pietsch, and finds also some analogies in other contexts, as the Nakano duality theory for Riesz spaces ([73, Sect. 1.4]). A special mention must be done here to the so called Maurey-Rosenthal Theorems for factorization of operators between Banach function spaces through an operator $T: L^{p}(\mu) \rightarrow L^{q}(v)$ for two particular positive (scalar) measures (see $[\mathbf{2 3}, \mathbf{2 5}])$ under certain convexity/concavity requirements. As we will show in this work, our class of operators can be characterized by a sort of vector measure version of this factorization.

Among other things, the results are applied to get more information on some particular topics of the theory of Banach function spaces. These spaces are ideals of ( $\mu$-almost everywhere equal) measurable functions, and two alternative ways of defining them are available in the mathematical literature. The first one can be found in $[\mathbf{6 6}, \mathbf{1 0 7}]$, where a direct definition as a normed sublattice (ideal) of $L^{1}(\mu)$ is given. The second one is given in $[\mathbf{8}, \mathbf{7 3}, \mathbf{9 8}, \mathbf{1 0 8}]$, where the definition by means of a so called function norm is presented. Both definitions are equivalent when we restrict our attention to the case of Banach spaces, whenever the order continuity and the Fatou properties are considered as 
separated properties not implicitly assumed in the definition (see for example $[\mathbf{8}, \mathrm{Ch} .1$ Defn. 1.1]). In the presentation of our results, we have chosen the first option in the version that can be found in [79, Rem. 2.3(ii)], where the quasi-Banach case is included. This is useful for us, since in general the $p$-th power of a Banach function space is not a Banach but a quasi-Banach space, and so we will need this case very often.

The reason for considering mainly Banach function spaces over a finite measure space is the same. In this case, such a Banach function space is always continuously contained in its (quasi-Banach) $p$-th power space. Moreover, the spaces of vector measure integrable functions appearing in a natural way in our results are Banach function spaces over a Rybakov measure for the corresponding vector measure.

The possibility of extending operators defined on a Banach function space to some sort of maximal or optimal domain - but, keeping the codomain space fixed - has been studied in various contexts within the general theory of kernel operators or differential operators; see for example $[6,17,65,77,100,101]$ and the references therein. See also $[4,10,11,85,99,103]$ for Hardy integral inequalities. In more recent years this idea has also turned out to be fruitful in the investigation of other large classes of operators (in addition to kernel and differential operators), such as convolutions, the Fourier transform and the Sobolev embedding; see $[18,20,21,34,75,80,81,86]$.

The contents of this memoir are organized as follows. After some introductory results that are presented in Chapter 1, in Chapter 2 we develop the theory of what we call the Köthe $p$-dual $X(\mu)^{p}$ of a Banach function space $X(\mu)$. This is the space of multipliers from $X(\mu)$ to $L^{p}(\mu)$, and we introduce and study it because it is a useful substitute of the ordinary Köthe dual space when the dual of the $p$-th power $X(\mu)_{[p]}$ is required. In Chapter 3 we analyze the problem of finding the best range of an operator $T: E \rightarrow X(\mu)$, i.e. the smallest Banach function space in which the range of an operator lies. In order to do this, we construct the duality of the well known theory of optimal domains and their characterization by means of spaces of integrable functions with respect to a vector measure ([79, Ch. 4]). Although some applications of these results are already given, we use this case as an introduction to the setting of $(p, q)$-th power factorable operators that is analyzed in Chapter 4 .

Thus, in Chapter 4 we develop the theory of $(p, q)$-th power factorable operators and we show that they can be associated to the optimality of the spaces between which the operator is defined. Let $X, Y, Z, W$ be Banach spaces, and let $T: X \rightarrow Y$ be an operator belonging to a class of operators $\mathscr{C}$. Roughly speaking, an operator $\widehat{T}: Z \rightarrow W$ in $\mathscr{C}$ gives an optimal factorization for $T$, if $X \subseteq Z$ and $W \subseteq Y$, and $Z$ is the biggest space (fixing $W$ ) and $W$ is the smallest space (fixing $Z$ ) such that $T$ can be factored in this way with a factorization map belonging to $\mathscr{C}$. This, in our case, can be translated in the following terms: if $Z \subseteq G$ and $\widehat{T}: Z \rightarrow W$ can be extended to $G$ with an operator $\widehat{T} \in \mathscr{C}$, then $Z=G$, and the same must happen with the adjoint $\widehat{T}^{*}$ and $W^{*}$. In case that the spaces involved $X, Y, Z, W$ 
are Banach function spaces with adequate properties, we will show that the optimal factorization for the class of the $(p, q)$-th power factorable operators can be constructed in a systematic way through a space $L^{p}\left(m_{R}\right)$ of $p$-integrable functions with respect to a vector measure and the dual of a space $L^{q}\left(m_{S^{*}}\right)$ of $q$-integrable functions with respect to other vector measure, where $T=S \circ R$ is a given factorization for $T$.

The final part of the thesis is devoted to show applications of the results obtained in the previous ones. Chapter 5 contains two parts. The first one deals with some applications in the setting of the interpolation theory for Banach function spaces, that has been recently developed specifically for the case of the spaces of $p$-integrable functions with respect to a vector measure (see $[\mathbf{2 6}, \mathbf{3 6}, \mathbf{3 7}, \mathbf{4 0}])$. In particular, we show that the properties of $p$-th and $(p, q)$-th power factorability are inherited by Calderón complex interpolation. The second part provides an analysis of the theory of kernel operators (Hille-Tamarkin) from the point of view of their factorization properties, and some optimality results are given. We provide also examples of how to apply these results to the study of some relevant kernel operators as convolution, Volterra, Hardy and Hartley operators. Finally, in Chapter 6 we center our attention in the problem of the optimal domain, optimal range and optimal factorizations for the Laplace transform, in order to show some concrete new results that are obtained by using our technique. 


\section{CHAPTER 1}

\section{PRELIMINARIES AND NOTATION}

In this chapter we establish the concepts used throughout the memoir on Banach lattices and specially Banach function spaces. We also collect useful results on factorization of operators and briefly recall the theory of integration of real functions with respect to vector measures defined on $\sigma$-algebras. All along in this memoir all the operators are considered linear and continuous.

\subsection{Measures and spaces}

We begin with some quite standard terminology and notations. A $\sigma$-algebra $\Sigma$ is a collection of subsets of a set $\Omega$ containing $\Omega$ which is closed for the difference and the numerable union, a pair $(\Omega, \Sigma)$ is a measurable space and an element of $\Sigma$ is a measurable element of the $\sigma$-algebra. The variation of a scalar measure $\mu$ is defined by

$$
|\mu|(A):=\sup \left\{\sum_{B \in \pi}|\mu(B)|: \pi \subset \Sigma \text { partition of } \mathrm{A}, \operatorname{card}(\pi)<\infty\right\}, \quad A \in \Sigma .
$$

Given a scalar (positive) measure $\mu$ and a measurable space the triad $(\Omega, \Sigma, \mu)$ is a measure space. We say that $A \in \Sigma$ is $\mu$-null if $|\mu|(A)=0$. Let $\mathscr{N}_{0}(\mu)$ denote the set of all $\mu$-null sets. We say that a measure $\mu$ is complete if every subset of every null set is measurable. We say that $\mu$ is a positive scalar measure if $0 \leq \mu(A) \leq \infty$ for every $A \in \Sigma$. If $\mu$ is a positive scalar measure, then $\mu=|\mu|$.

1.1.1. Vector measures. For a real Banach space $E$ a vector measure is a $\sigma$-additive (countably additive) mapping from $\Sigma$ taking values in $E, m: \Sigma \rightarrow E$. The variation of a vector measure $m$ is defined as

$$
|m|(A):=\sup \left\{\sum_{B \in \pi}\|m(B)\|_{E}: \pi \subset \Sigma \text { partition of } \mathrm{A}, \operatorname{card}(\pi)<\infty\right\}, \quad A \in \Sigma .
$$

It is said that $m$ has finite variation if $|m|(\Omega)<\infty$, and $\sigma$-finite variation if there exists a sequence of subsets $\left\{A_{i}\right\}_{i \in \mathbb{N}} \subseteq \sum$ such that $\Omega=\cup_{i \in \mathbb{N}} A_{i}$ and $|m|\left(A_{i}\right)<\infty$ for every $i \in \mathbb{N}$.

By semivariation of $m$ we mean the set function $\|m\|: \Sigma \rightarrow[0, \infty)$ defined by

$$
\|m\|(A):=\sup \left\{\left|\left\langle m, x^{*}\right\rangle\right|(A):\left\|x^{*}\right\|_{E^{*}} \leq 1\right\} \quad A \in \Sigma .
$$


The null sets of $m$ are those $A \in \sum$ such that $\|m\|(A)=0$, these sets are briefly called $m$-null sets. Recall that a Rybakov measure is a scalar measure defined as $\left\langle m, x^{*}\right\rangle(A)=\left\langle m(A), x^{*}\right\rangle$, where $x^{*} \in E^{*}$ and $A \in \Sigma$ and it is such that $\|m\| \ll\left|\left\langle m, x^{*}\right\rangle\right|$. Such a measure always exists (see [31, Th. IX.2.2]).

A simple function is a function that takes a finite number of values and it can be written as $s=\sum_{i=1}^{n} \alpha_{i} \chi_{A_{i}}$ where $\left\{\alpha_{i}\right\}_{i=1}^{n} \subset \mathbb{R}$ and $\left\{A_{i}\right\}_{i=1}^{n} \subseteq \Sigma$ is a partition of $\Omega$. $\mathscr{S}(\Sigma)$ will be the set of all simple functions. Recall that the Borel sets of a topological space are the members of the $\sigma$-algebra generated by the open sets. We will assume, without explicit mention, that the measure spaces considered are complete. In this way, a scalar function is Borel measurable (the preimage of every Borel subset of scalars is measurable) if and only if equals almost everywhere to the limit of a sequence of simple functions. Following the notation of [56] or [69, Defn. 23.1 and Ex. 23.3(iv)] we denote by $L^{0}(\mu)$ the space of classes of real-valued measurable functions modulo equality $\mu$-almost everywhere, which is a complete, metrizable and not normable topological vector space (see e.g. [79, Lem. 2.1]). If $m$ is a vector measure, consider the space $L^{0}(m)$ of equivalence classes of measurable functions which differ only in a $m$-null set. If $\left|\left\langle m, x^{*}\right\rangle\right|$ is a Rybakov measure, then $L^{0}(m)=L^{0}\left(\left|\left\langle m, x^{*}\right\rangle\right|\right)$. When we consider a measurable space $(\Omega, \Sigma)$ and measures $\mu, \lambda: \Sigma \rightarrow[0, \infty]$ such that $\lambda \ll \mu$ it is defined the map $[i]: L^{0}(\mu) \rightarrow L^{0}(\lambda)$ by $[i]\left([f]_{\mu}\right):=[f]_{\lambda}$, where $[\cdot]_{\mu}$ and $[\cdot]_{\lambda}$ denote the respective classes modulo equal $\mu$ and $\lambda$-almost everywhere, respectively. Let us denote this inclusion by “ $\hookrightarrow_{[i]}$ ”. This map is called inclusion/quotient map and is well defined (see e.g. [13, p. 90]). These notions with their theoretic developments can be found e.g. in $[\mathbf{3 1}, \mathbf{3 2}, \mathbf{4 5}, \mathbf{7 9}])$.

1.1.2. Banach lattices and Banach function spaces. A (real) Banach lattice is a (real) Banach space endowed with an order (i.e. a reflexive, antisymmetric and transitive binary relation) denoted by " $\leq$ " or " $<$ ", that is compatible with the sum and the multiplication by a non-negative scalar. Given a Banach lattice, the least upper bound is denoted by " $\vee$ " and the greatest lower bound is denoted by " $\wedge$ ". If $X$ is a Banach lattice and $x \in X$, the absolute value of $x$ is defined as $|x|:=x \vee(-x)$, the positive part of $x$ as $x^{+}:=x \vee 0$ and the negative part as $x^{-}:=-(x \wedge 0)$. Observe that $|x|=x^{+}+x^{-}$and that $x=x^{+}-x^{-}$. Let us define $X^{+}$the positive cone as the set of all positive parts, and $X^{-}$the negative cone the set of all negative parts of the elements of $X$. An advantage property of Banach lattices versus Banach spaces is that an equality or inequality involving a finite number of elements holds if and only if

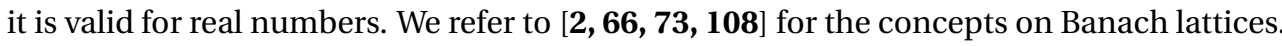

Let $(\Omega, \Sigma, \mu)$ be a $\sigma$-finite measure space that we assume complete. A Banach function space over $\mu$ (or over $(\Omega, \Sigma, \mu)$ ), denoted by $X(\mu)$, is a Banach space of classes modulo $\mu$ almost every where of functions locally $\mu$-integrable such that

(I) a measurable function $f \in X(\mu)$, whenever there exists $g \in X(\mu)$ such that $|f| \leq$ $|g| \mu$-almost everywhere, in that case $\|f\|_{X(\mu)} \leq\|g\|_{X(\mu)}$, and

(II) $\chi_{A} \in X(\mu)$ for all $A \in \Sigma$ such that $\mu(A)<\infty$. 
All along this memoir, unless otherwise stated, we assume that the Banach function spaces are based over a finite measure. In this way $X(\mu)$ is a Banach lattice with the pointwise order $\mu$-almost everywhere. If the measure $\mu$ is clear in the context we simply write $X$ instead of $X(\mu)$. This definition can be found in [66, Defn. 1.b.17], for $\sigma$-finite measures. The approach by means of function norms can be found in [107, Sect. 30] or in [69, Ch. $1 \S 9$ ] and the equivalence with our definition in [79, Rem. 2.3(ii)]. We can find some properties in [79, Prop. 2.2], for example $L^{\infty} \subseteq X$ - since $\mu$ is finite-, $X \subseteq L^{0}$ continuously with the topology of convergence in measure and $\chi_{\Omega}$ is a weak order unit, i.e. $f \wedge n \chi_{\Omega} \rightarrow f$ pointwise for every $f \in X^{+}$. In [98, Defn. 13.1] and [69, Ch.1 Sect. 9] there are other equivalent definitions.

We denote by $X^{\prime}$ the Köthe dual space of $X$, i.e. the Banach function space of all integral functionals in $X^{*}$-the (topological) dual space-, so $X^{\prime} \subseteq X^{*}$ and we denote by $\kappa: X^{\prime} \hookrightarrow X^{*}$ the isometric inclusion. The duality between a Banach function space $X$ and its dual space $X^{*}=\mathscr{L}(X, \mathbb{R})$ is denoted by $\langle f, \xi\rangle$, where $f \in X$ and $\xi \in X^{*}$. If $X$ is a Banach function space, $X^{*}$ is a Banach lattice and $X^{\prime}$ is a Banach function space (see e.g. [79, Lem. 2.8(i) and Prop. 2.16]). Notice that every function $f$ in a Banach function space $X$ is locally integrable by definition, thus $X \subseteq L^{1}$, since $X^{\prime}$ is a Banach function space and we may apply Hölder-Rogers inequality to $f \chi_{\Omega}$. If $E$ is a Banach space and $X$ is a Banach function space and $T: E \rightarrow X$ is an operator, i.e. a linear and norm continuous map, we write $T^{*}$ for its adjoint operator and $T^{\prime}$ for its Köthe adjoint operator, i.e. for the restriction of $T^{*}$ to the Köthe dual $X^{\prime}$. A quasi-norm is likewise a norm which do not satisfies the triangular inequality, which is replaced by $\|x+y\| \leq C(\|x\|+\|y\|)$ for $x, y \in X$ and some $C \geq 1$. A quasi-Banach function space is defined as in the case of a Banach function space by replacing the norm by a quasi-norm. The same definitions that we use for Banach function spaces make sense. Sometimes, we will need to consider quasi-Banach function spaces, which are complete vector lattices of measurable functions whose topology is given by means of a quasi-norm instead of a norm.

Let $X$ be a Banach function space. We say that $X$ is order continuous if for every sequence $\left(f_{n}\right)_{n} \subset X^{+}$such that $f_{n} \downarrow 0$ we have that the sequence of the norms also converges to zero, i.e. $\left\|f_{n}\right\| \downarrow 0$. Often it is said order continuous when the limit is defined by nets and $\sigma$-order continuous when it is defined by sequences. Thanks to [79, Rem. 2.5], both concepts coincide for Banach function spaces, hence the definition above makes sense. We say that $X$ has the Fatou property or simply that it is Fatou if for all sequences $\left(f_{n}\right)_{n} \subset X^{+}$ such that $f_{n} \uparrow f, f$ is measurable and $\sup _{n}\left\|f_{n}\right\|<\infty$, then $f \in X$ and $\lim _{n}\left\|f_{n}\right\|=\|f\|$. In [66, p.29-30] we can find the following characterizations: $X$ is order continuous if and only if $X^{\prime}=X^{*}$ and $X$ is Fatou if and only if $X^{\prime \prime}=X$. In this last case we say that $X$ is a perfect space. Observe that in case that $X$ is order continuous, then $\mathscr{S}(\Sigma)$ is dense in $X([\mathbf{7 9}$, Rem. 2.6]). An operator $T: X \rightarrow Y$ between Banach function spaces is order continuous if for each sequence $\left(f_{n}\right)_{n}$ of positive functions such that $f_{n} \rightarrow 0$ pointwise almost everywhere, we have that $T f_{n} \rightarrow 0$ in norm. Observe that a Banach function space is order continuous 
if the identity operator of $X$ is order continuous. More useful facts on order continuity can be found, for example, in [66, p.32], [3, §1.4], [98].

Consider a pair $X$ and $Y$ of quasi-Banach function spaces and $1 \leq p \leq \infty$. An operator $T: X \rightarrow Y$ is $p$-convex if there exists $K_{p}<\infty$ such that for all $n \in \mathbb{N}$ and for all $\left\{f_{1}, \ldots, f_{n}\right\} \subseteq$ $X$,

or

$$
\left\|\left(\sum_{j=1}^{n}\left|T f_{j}\right|^{p}\right)^{1 / p}\right\|_{Y} \leq K_{p}\left(\sum_{j=1}^{n}\left\|f_{j}\right\|_{X}^{p}\right)^{1 / p}, \quad \text { if } p \in[1, \infty)
$$

$$
\left\|\bigvee_{j=1}^{n}\left|T f_{j}\right|\right\|_{Y} \leq K_{\infty} \max _{j=1, \ldots, n}\left\|f_{j}\right\|_{X}, \quad \text { if } p=\infty
$$

For $1 \leq q<\infty$, an operator $T: X \rightarrow Y$ is $q$-concave if there exists $K^{q}<\infty$ such that for all $n \in \mathbb{N}$ and for all $\left\{f_{1}, \ldots, f_{n}\right\} \subseteq X$,

$$
\left(\sum_{j=1}^{n}\left\|T f_{j}\right\|_{Y}^{q}\right)^{1 / q} \leq K^{q}\left\|\left(\sum_{j=1}^{n}\left|f_{j}\right|^{q}\right)^{1 / q}\right\|_{X}, \quad \text { if } q \in[1, \infty) .
$$

A quasi-Banach function space $X$ is $p$-convex (respectively $q$-concave) if the identity map on $X$ is $p$-convex (respectively $q$-concave). Every operator and every Banach function space is 1-convex.

The following definitions can be found in [66, Defn. 1.e.12] and also in [24, Sect. 7.7]. The Rademacher functions $r_{k}$ are defined for $t \in[0,1]$ by $r_{k}(t):=\operatorname{sign}\left(\sin 2^{k} \pi t\right)$, thus in each subinterval we have

$$
r_{k}(t)=(-1)^{j}, \quad t \in\left[\frac{j}{2^{k}}, \frac{j+1}{2^{k}}\right), j=0, \ldots, 2^{k}-1, k=1,2,3, \ldots .
$$

Let $E$ be a Banach space. We say that $E$ has type $p \in[1,2]$ if there exists $C \geq 0$ such that for every $x_{1}, \ldots, x_{n} \in E$

$$
\left(\int_{0}^{1}\left\|\sum_{k=1}^{n} r_{k}(t) x_{k}\right\|_{E}^{2} d t\right)^{1 / 2} \leq C\left(\sum_{k=1}^{n}\left\|x_{k}\right\|_{E}^{p}\right)^{1 / p} .
$$

It is said that $E$ has cotype $q \in[2, \infty]$ if there exists $C \geq 0$ such that for every $x_{1}, \ldots, x_{n} \in E$

$$
\left(\sum_{k=1}^{n}\left\|x_{k}\right\|_{E}^{q}\right)^{1 / q} \leq C\left(\int_{0}^{1}\left\|\sum_{k=1}^{n} r_{k}(t) x_{k}\right\|_{E}^{2} d t\right)^{1 / 2} .
$$

Every Banach space has cotype $\infty$ and type 1 [66, p. 73].

1.1.3. $L^{p}(m)$ spaces and optimal domains. Let us now regard vector measures and consider the space $L^{p}(m)$ of $p$-integrable functions with respect to a vector measure $m$. If $1 \leq p \leq \infty$, we will write $p^{\prime}$ for the extended real number satisfying $1 / p+1 / p^{\prime}=1$. An element $f \in L^{0}(m)$ is weakly integrable with respect to $m$ if it is integrable with respect to each scalar measure $\left|\left\langle m, x^{*}\right\rangle\right|$ for $x^{*} \in E^{*}$. If moreover for each $A \in \Sigma$ there is a unique vector denoted by $\int_{A} f d m \in E$ such that

$$
\int_{A} f d\left\langle m, x^{*}\right\rangle=\left\langle\int_{A} f d m, x^{*}\right\rangle,
$$


for each $x^{*} \in E^{*}$ —sometimes called the barycentric equality—, we say that $f$ is integrable with respect to $m$ ( $m$-integrable). The space of all $m$-integrable functions is denoted by $L^{1}(m)$, which is a Banach lattice when we consider the $m$-almost everywhere pointwise order. Such a function $f \in L^{0}(m)$ is $p$-integrable with respect to $m$ if $|f|^{p}$ is integrable with respect to $m, 1 \leq p<\infty$. The expression

$$
\|f\|_{L^{p}(m)}:=\sup _{x^{*} \in B_{E^{*}}}\left(\int_{\Omega}|f|^{p} d\left|\left\langle m, x^{*}\right\rangle\right|\right)^{1 / p},
$$

that is well defined for each measurable function $f$, defines in fact a function norm on $L^{0}(m)$. It is equivalent to the expression

$$
\|f\|_{L^{p}(m)}:=\sup _{A \in \Sigma}\left\|\int_{A}|f|^{p} d m\right\|_{E}^{1 / p} .
$$

The space $\left(L^{p}(m),\|\cdot\|_{L^{p}(m)}\right)$ is a $p$-convex order continuous Banach function space over each Rybakov measure for $m$. The integration map $f \in L^{1}(m) \rightsquigarrow \int f d m \in E$ is always continuous.

Let $X$ be a quasi-Banach function space over a finite measure, let $E$ be a Banach space and $T: X \rightarrow E$ an order continuous operator. Then we define the vector measure associated with $T, m_{T}: \Sigma \rightarrow E$, is $m_{T}(A):=T\left(\chi_{A}\right)$ for every $A \in \Sigma$. Note that if $X$ is order continuous and $T$ is norm continuous, then $T$ is order continuous. Assuming that $X$ is based on $\mu$, then $\mathscr{N}_{0}(\mu) \subseteq \mathscr{N}_{0}\left(m_{T}\right)$. We say that $T$ is $\mu$-determined if $m_{T}$ and $\mu$ have the same null sets. Let us show some results related to such spaces. Here we have some useful properties for the measure associated with an operator.

Proposition 1.1 ([79, Prop. 4.4]). Let $X$ be an order continuous quasi-Banach function space on a finite measure $\mu$. Let $E$ be a Banach space and let $T: X \rightarrow E$ be an operator. Then the following statements hold for the associated vector measure $m_{T}: \Sigma \rightarrow E$ :

(1) If $f \in X$, then $f \in L^{1}\left(m_{T}\right)$ and $\int_{A} f d m_{T}=T\left(f \chi_{A}\right)$.

(2) The vector measure $m_{T} \ll \mu$, so the inclusion/quotient map $[i]: X \rightarrow L^{1}\left(m_{T}\right)$ is well defined, linear continuous and $\|[i]\|=\|T\|$.

(3) In case that $m_{T} \equiv \mu$ (i.e. they have the same null sets) the space $L^{1}\left(m_{T}\right)$ is a Banach function space based on $\mu$ that embeds $X$ by means of the map $[i]$, and the integration map $I_{m_{T}}: L^{1}\left(m_{T}\right) \rightarrow E$ is the unique extension of $T$ to its optimal domain.

Theorem 1.2 ([79, Th. 4.14]). Let $X$ be a order continuous quasi-Banach function space over a finite measure space $(\Omega, \Sigma, \mu)$. Let $T: X \rightarrow E$ be a Banach space valued operator. Suppose that $T$ is $\mu$-determined. Then the order continuous Banach function space $L^{1}\left(m_{T}\right)$ is the optimal domain among all quasi-Banach function spaces with order continuous quasinorm (based on $(\Omega, \Sigma, \mu)$ ).

1.1.4. The $p$-th power space. Let $X$ and $Y$ be two quasi-Banach function spaces over $\mu$. Given $g \in L^{0}$, if $f g \in X$ for every $f \in X$ the map $M_{g}(f)=f g \in Y$ is well defined for all 
$f \in X$, it is said that $M_{g}$ is a multiplier operator from $X$ to $Y$, which is always continuous. Following the notation of [71] and [12], the space of multipliers from $X$ to $Y$ is denoted as

$$
X^{Y}:=\left\{g \in L^{0}: g \cdot X \subseteq Y\right\} .
$$

We can define $\|g\|_{X^{Y}}:=\sup _{f \in B_{X}}\|g f\|_{Y}$ that is a complete norm in this space, since $X$ has weak order unit by definition and comments above, so $X^{Y}$ is a Banach space. Let us note that $X^{\prime}=X^{L^{1}}$. Observe that this definition makes sense also if $Y$ is a Banach function space over another measure $v$, such that $v \ll \mu$. Let $1 \leq r<p \leq \infty$ and $q \geq 1$ be such that $\frac{1}{r}=\frac{1}{p}+\frac{1}{q}$, then $L^{q}=\left(L^{p}\right)^{L^{r}}$ (see [71, Prop. 3]), despite it can be the trivial space (e.g. $\left(L^{r}\right)^{L^{s}}=\{0\}$ for $\left.r<s\right)$. In [97, Th. 1.8] we can find the following condition: if $X$ and $Y$ are Banach function spaces based on a non-atomic measure and the following inequality holds

$$
\inf \{p \geq 1: X \text { is } p \text {-concave }\}<\sup \{p \geq 1: Y \text { is } p \text {-convex }\},
$$

then $X^{Y}=\{0\}$. For more information, including sufficient conditions to ensure that the space of multipliers between Banach lattices is a Banach function space can be found in [13], [71] and [79, Ch.2].

There are several results involving factorization through multiplier operators, for example the so-called Maurey-Rosenthal's Theorems.

Theorem 1.3 (Maurey-Rosenthal, see [23, Cor. 2]). For $1 \leq r<\infty$ let $T$ be an $r$-convex linear operator from a quasi-Banach space $E$ into an $r$-concave quasi-Banach function space $Y$. Then $T$ factors through $L^{r}$ as follows:

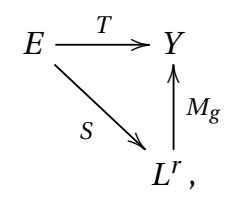

where $S$ is an operator and $0 \leq g \in\left(Y^{\prime}\right)^{L^{r^{\prime}}}$.

Theorem 1.4 (Maurey-Rosenthal, see [23, Cor. 5]). Let $1 \leq r<\infty$ let $T$ be an $r$-concave operator from an $r$-convex and order continuous quasi-Banach function space $X$ into a quasi-Banach space E. Then $T$ factors through $L^{r}$ as follows:

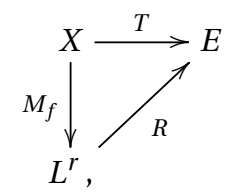

where $R$ is an operator and $0 \leq f \in X^{L^{r}}$.

For $0<p<\infty$, the $p$-th power space $X_{[p]}$ of $X$ is defined as

$$
X_{[p]}:=\left\{f \in L^{0}:|f|^{1 / p} \in X\right\} .
$$


This space is a quasi-Banach function space over $\mu$ when it is endowed with the quasinorm $\|f\|_{[p]}:=\left\||f|^{1 / p}\right\|_{X}^{p}$ ([79, Prop. 2.22]). Observe that if $X$ is a Banach lattice, then the quasi-norm defined for $X_{[p]}$ for $1 \leq p<\infty$ is a $1 / p$-norm, i.e.

$$
\|f+g\|_{[p]}^{1 / p} \leq\|f\|_{X_{[p]}}^{1 / p}+\|g\|_{X_{[p]}}^{1 / p}, \quad f, g \in X_{[p]} .
$$

It is not always true that a quasi-norm is continuous for the associated topology. If a quasinorm $\|\cdot\|$ is $r$-subadditive for some $r \geq 0$, i.e. $\|x+y\|^{r} \leq\|x\|^{r}+\|y\|^{r}$ for some $r>0$, then it is continuous, i.e. if $\left(x_{n}\right) \rightarrow x$ then $\left\|x_{n}\right\| \rightarrow\|x\|$. Aoki-Rolewicz's Theorem [92] asserts that every quasi-norm has an $r$-subadditive quasi-norm for some $r>0$ that defines the same topology. The main basic properties of this space can be found in [79, Ch. 2 Sect. 2].

Proposition 1.5. Let $X$ be a quasi-Banach function space over a finite measure $\mu$.

(1) Let $0<p, q<\infty$. Then $X_{[p]}[q]=X_{[p q]}$.

(2) Let $0<p<\infty$. If $Y$ is a quasi-Banach function space over $\mu$ we have that $X \subseteq Y$ if and only if $X_{[p]} \subseteq Y_{[p]}$.

(3) Let $q, r, s>0$ be such that $q=r+s$. Then $\|f g\|_{X_{[q]}} \leq C\|f\|_{X_{[r]}}\|g\|_{X_{[s]}}$ for all $f \in X_{[r]}$ and $g \in X_{[s]}$.

(4) Let $0<p<\infty$. Then $X_{[p]}$ is a quasi-Banach function space.

(5) Let $0<p<\infty$. Then $X$ is order continuous if and only if $X_{[p]}$ is order continuous.

(6) If $0<p \leq q<\infty$ we have that $X_{[p]} \subseteq X_{[q]}$, in particular $X=X_{[1]} \subseteq X_{[p]}$ for all $1 \leq p<\infty$ and $X_{[p]} \subseteq X$ for all $0<p \leq 1$.

(7) Let $0<p \leq 1$. If $X$ is a Banach function space, so is $X_{[p]}$.

(8) Let $1 \leq p<\infty$. If $X$ is a $p$-convex Banach function space, then $X_{[p]}$ is a Banach function space.

(9) The following statements are equivalent

(a) $X=L^{\infty}$.

(b) $X=X_{[p]}$ for some $p \neq 1$. In that case it holds for all $p \in(0, \infty)$.

(c) $X$ is an algebra of functions with the ( $\mu$-almost everywhere) pointwise multiplication.

(10) $\left(X^{Y}\right)_{[p]}=\left(X_{[p]}\right)^{Y_{[p]}}$ for $p \in(0, \infty)$.

(11) $\left(X_{[p]}\right)^{\prime}=\left(X^{L^{p}}\right)_{[p]}$ for $p \in(0, \infty)$.

All along this research will be important to find a representation formula for the dual of the space $X_{[p]}$, so the property (11) above plays an important role. For a proof of this last statement see e.g. [79, Prop. 2.29], [71, Sect. 2(g)] or [97, Th. 3.2]. We note that, since $X^{L^{p}}$ is $p$-convex with constant 1 (see [12, Th. 5.1] or [97, Prop. 3.1]), then $\left(X^{L^{p}}\right)_{[p]}$ is a Banach function space (see in [79, Prop. 2.23(iii)]). However, if $X$ is not $p$-convex we cannot assure that $X_{[p]}$ is again a Banach function space, in consequence it may happens that $\left(X_{[p]}\right)^{\prime}=$ $\{0\}$. It does not necessarily imply that $\left(X^{p}\right)_{[p]} \neq\left(X_{[p]}\right)^{\prime}$, for instance, $L^{p}[0,1]_{[q]}=L^{p / q}[0,1]$ has trivial dual when $p<q$, in this case $\left(L^{p}[0,1]\right)^{q}=\{0\}$ and so its $q$-th power space is also trivial. In conclusion, in virtue of these arguments and (1.7), in order to work with non 
trivial spaces, usually we will assume that $X$ is $p$-convex, since often the measures that we will use are non-atomic.

\subsection{Operators}

The following fact was shown for a more general case in [79, Lem. 2.21]. Let $1 \leq p<\infty$, let $X$ be a Banach function space and let $f \in X$. Let us define $g:=\frac{f}{\|f\|_{X}}$ and $h:=\frac{\chi_{\Omega}}{\left\|\chi_{\Omega}\right\|_{X_{[p-1]}}}$. By the Young inequality we have

$$
\|g h\|_{X_{[p]}}=\left\||g|^{1 / p}\left(|h|^{p^{\prime} / p}\right)^{1 / p^{\prime}}\right\|_{X}^{p} \leq\left(\frac{1}{p}\|g\|_{X}+\frac{1}{p^{\prime}}\left\||h|^{p^{\prime} / p}\right\|_{X}\right)^{p}=\left(\frac{1}{p}+\frac{1}{p^{\prime}}\|h\|_{X_{[p-1]}}^{1 /(p-1)}\right)^{p}=1 .
$$

Therefore $\|f\|_{X_{[p]}}=\left\|f \chi_{\Omega}\right\|_{X_{[p]}} \leq\left\|\chi_{\Omega}\right\|_{X_{[p-1]}}\|f\|_{X}$. So if $i_{[p]}: X \hookrightarrow X_{[p]}$ denotes the inclusion map, then $\left\|i_{[p]}\right\| \leq\left\|\chi_{\Omega}\right\|_{X}^{p-1}$.

1.2.1. The $p$-th power factorable operators. The following definition was introduced in [79, Defn. 5.1].

Definition 1.6. Let $1 \leq p<\infty$ and $X$ be an order continuous Banach function space based on a finite measure, and let $E$ be a Banach space. An operator $T: X \rightarrow E$ is $p$-th power factorable, if there exists an operator $T_{[p]}: X_{[p]} \rightarrow E$, such that $\left.T_{[p]}\right|_{X}=T$. In other words, if $i_{[p]}$ denotes the natural inclusion and $T_{[p]}$ the extension of $T$ to its $p$-th power space, then $T=T_{[p]} \circ i_{[p]}$, i.e. the following diagram commutes

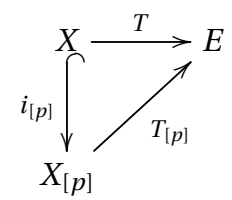

DiAgram 1.1. Definition of $p$-th power factorable operator

We observe that this extension $T_{[p]}$ must be unique, since $i_{[p]}$ is continuous and has dense range. The main characterization for this definition follows from the density of $X$ in $X_{[p]}$ for $p \geq 1$, since the base measure is finite and $X$ is assumed to be order continuous, i.e. there is a constant $C>0$ such that $\|T f\|_{E} \leq C\left\||f|^{1 / p}\right\|_{X}^{p}=C\|f\|_{X_{[p]}}$, for every $f \in X$ (see Theorem 1.7 below).

One of the aims of our research is to find factorization schemes. In this sense we will greatly use factorization theorems as the followings. The main factorization that we investigate is the optimal domain factorization, from the point of view of the $p$-th power factorability. 
Theorem 1.7 ([79, Th. 5.7]). Given are $1 \leq p<\infty$, an order continuous quasi-Banach function space $X$ based on a finite measure space $(\Omega, \Sigma, \mu)$, and a Banach space $E$. The following assertions for a $\mu$-determined operator $T: X \rightarrow E$ are equivalent.

(1) $T$ is $p$-th power factorable.

(2) There is a constant $C>0$ such that

$$
\|T f\|_{E} \leq C\|f\|_{X_{[p]}}=C\left\||f|^{1 / p}\right\|_{X}^{p}, \quad f \in X \subseteq X_{[p]} .
$$

(3) $X \subseteq L^{p}\left(m_{T}\right)$.

(4) $X_{[p]} \subseteq L^{1}\left(m_{T}\right)$.

(5) For every $x^{*} \in E^{*}, d\left\langle m_{T}, x^{*}\right\rangle / d \mu \in\left(X_{[p]}\right)^{\prime}$.

Let us illustrate how the indexes work for a $p$-th power factorable operator $T: X \rightarrow E$ with respect to the $p$-integrability. Let $1 \leq q \leq p<\infty$. Under the requirements of the theorem above, we have two different factorization schemes
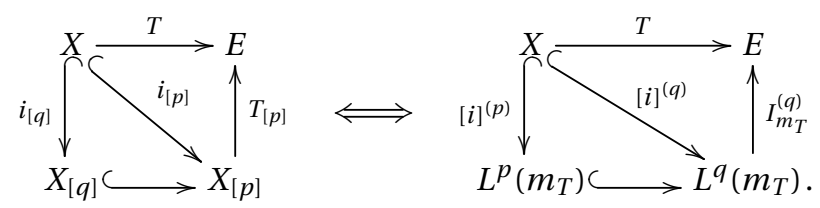

DIAGRAM 1.2. $p$-th power factorization versus factorization through optimal domains

If $X$ is an order continuous Banach function space based on a finite measure $\mu$ and $T: X \rightarrow E$ is a Banach space-valued operator, we know that $m_{T}(A):=T\left(\chi_{A}\right)$ is a vector measure. For the case of $p$-th power factorable operators, if $T$ is $\mu$-determined, the inclusion/quotient map $[i]: X \rightarrow L^{p}\left(m_{T}\right)$ is injective. Since $\left\|m_{T}\right\| \ll \mu$ we always can consider $f \in X$ as a function in $L^{1}\left(m_{T}\right)$, this can be deduced from the density of the simple functions and the Dominated Convergence Theorem. Moreover $\int_{\Omega} f d m_{T}=T(f)$, let us rewrite this with the equivalence classes, i.e. $T\left([f]_{\mu}\right)=\int_{\Omega}[f]_{m_{T}} d m_{T}=\int_{\Omega}[i]\left([f]_{\mu}\right) d m_{T}=$ $I_{m_{T}} \circ[i]\left([f]_{\mu}\right)$. If $\mu$ is not absolutely continuous with respect to $m_{T}$, the inclusion/quotient map cannot be injective. Without the injectivity assumption, taking into account that 1 -th power factorability coincides with continuity, the map $[i]$ is still well defined and continuous, and we always have that $[i](X) \subseteq L^{1}\left(m_{T}\right)$ continuously (since $\left\|m_{T}\right\| \ll \mu$ ), i.e. $X \hookrightarrow_{[i]} L^{1}\left(m_{T}\right)$. In the case that we can assure that in fact $[i](X)$ is included in the subspace $L^{p}\left(m_{T}\right)$ of $L^{1}\left(m_{T}\right)$ for any $p>1$, we will also write $X \hookrightarrow_{[i]} L^{p}\left(m_{T}\right)$. The proof of the next result follows the ideas of [13].

Proposition 1.8. Let $1 \leq p<\infty$, and let $X$ be an order continuous Banach function space. Consider a Banach space $E$. The following assertions for an operator $T: X \rightarrow E$ are equivalent. 
(1) $T$ is $p$-th power factorable.

(2) $X \hookrightarrow_{[i]} L^{p}\left(m_{T}\right)$.

Proof. Assume (1). Obviously the extension $T_{[p]}: X_{[p]} \rightarrow E$ of $T$ to $X_{[p]}$ is unique since $X$ is dense in $X_{[p]}$ and $T$ is continuous. Note that $m_{T_{[p]}}=m_{T}$. By the comments above we can assure that $X_{[p]} \hookrightarrow_{[i]} L^{1}\left(m_{T_{[p]}}\right)=L^{1}\left(m_{T}\right)$. Finally for $f \in X$ we have that $|f|^{p} \in X_{[p]} \hookrightarrow[i] L^{1}\left(m_{T}\right)$, hence

$$
\|f\|_{L^{p}\left(m_{T}\right)}=\left\|\left(|f|^{p}\right)^{1 / p}\right\|_{L^{p}\left(m_{T}\right)}=\left\||f|^{p}\right\|_{L^{1}\left(m_{T}\right)}^{1 / p} \leq K^{1 / p}\left\||f|^{p^{1 / p}}\right\|_{[p]}^{1 / p}=K^{1 / p}\|f\|_{X} .
$$

So we have that $X \hookrightarrow_{[i]} L^{p}\left(m_{T}\right)$. For the converse suppose that $X \hookrightarrow_{[i]} L^{p}\left(m_{T}\right)$. Then the calculation above shows that $X_{[p]} \hookrightarrow[i] L^{1}\left(m_{T}\right)$. In consequence, $T=I_{m_{T}} \circ[i] \circ i_{[p]}$ (see [79, Prop. 4.4(i)]. Thus, for $f \in X$, we have that

$$
\|T f\|_{E}=\left\|\left(I_{m_{T}} \circ[i] \circ i_{[p]}\right) f\right\|_{E} \leq\left\|I_{m_{T}} \circ[i]\right\|\|f\|_{X_{[p]}} .
$$

This finishes the proof.

Theorem 1.9 ([39, Th. 5]). Let $X$ and $Y$ be Banach lattices. Let $n: \Sigma \rightarrow X$ and $m: \Gamma \rightarrow Y$ be positive vector measures, and let $p, q>1$. For an operator $T: L^{p}(n) \rightarrow\left(L^{q}(m)\right)^{\prime}$, the following statements are equivalent:

(1) There is a constant $K>0$ such that, for every finite set of functions $f_{1}, \ldots, f_{N} \in$ $L^{p}(n)$

$$
\left\|\left(\sum_{i=1}^{N}\left|T f_{i}\right|^{p}\right)^{1 / p}\right\|_{\left(L^{q}(m)\right)^{\prime}} \leq K\left\|\left(\sum_{i=1}^{N}\left|f_{i}\right|^{p}\right)^{1 / p}\right\|_{L^{p}(n)}
$$

(2) The operator $T$ factors as follows:

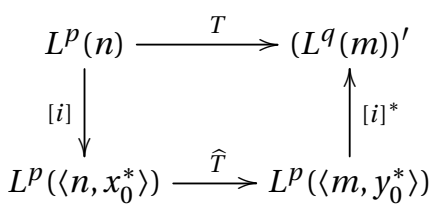

DiAgram 1.3. Factorizaton through $L^{p}$-spaces for $T: L^{p}(n) \rightarrow\left(L^{q}(m)\right)^{\prime}$

where $0 \leq x_{0}^{*} \in B_{X^{*}}, 0 \leq y_{0}^{*} \in B_{Y^{*}}$, [i] is the inclusion/quotient operator, $[i]^{*}$ is the adjoint of the inclusion/quotient map and $\widehat{T}$ is an extension of $T$.

Theorem 1.10 (Nikishin, see e.g. [106, Th. III.H.6] and [43, Cor. VI-2.7]). Let X be a Banach space of type $p$ and $(\Omega, \Sigma, \mu)$ a $\sigma$-finite measure space. Then every sublinear operator $T: X \rightarrow L^{0}(\mu)$ factors through $L^{p, \infty}(\mu)$. In other words, for a suitable operator $H$ and suitable $f \in X^{L^{p, \infty}(\mu)}$ we have 


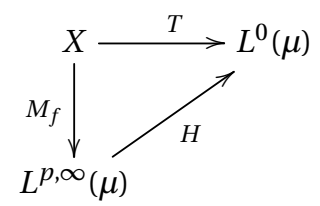

DiAgRAM 1.4. Nikishin's Theorem

Remark 1.11. The factorization of the Nikishin's Theorem above can be written in the particular case of an operator $T: L^{p} \rightarrow Y(\mu)$ defined on the $L^{p}$-space of a scalar measure as follows

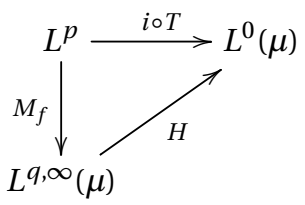

where $q=\min \{2, p\}$ and $i: Y(\mu) \rightarrow L^{0}(\mu)$ denotes the natural inclusion.

1.2.2. Kernel operators. Finally, we give the notation for the study of kernel operators. Let $\left(\Omega_{1}, \Sigma_{1}, \mu_{1}\right)$ and $\left(\Omega_{2}, \Sigma_{2}, \mu_{2}\right)$ be two finite measure spaces. A kernel function is a non-negative function $K \in L^{1}\left(\mu_{1} \otimes \mu_{2}\right)$. Consider a pair of Banach function spaces $X\left(\mu_{2}\right)$ and $Y\left(\mu_{1}\right)$, and assume that the formula

$$
\left(T_{K} f\right)\left(\omega_{1}\right):=\int_{\Omega_{2}} K\left(\omega_{1}, \omega_{2}\right) f\left(\omega_{2}\right) d \mu_{2}\left(\omega_{2}\right), \quad f \in X\left(\mu_{2}\right),
$$

defines a continuous map $T_{K}: X\left(\mu_{2}\right) \rightarrow Y\left(\mu_{1}\right)$. Then we say that $T_{K}$ is the kernel operator associated with $K$. Let $\omega_{1} \in \Omega_{1}$ and $\omega_{2} \in \Omega_{2}$, we define the functions $K\left(\cdot, \omega_{2}\right)\left(\varpi_{1}\right):=$ $K\left(\oplus_{1}, \omega_{2}\right)$ for $\varpi_{1} \in \Omega_{1}$ and $K\left(\omega_{1}, \cdot\right)\left(\oplus_{2}\right):=K\left(\omega_{1}, \oplus_{2}\right)$ for $\varpi_{2} \in \Omega_{2}$. Notice that $K\left(\omega_{1}, \cdot\right) \in$ $\left(X\left(\mu_{2}\right)\right)^{*}$, since $K \in L^{1}\left(\mu_{1} \otimes \mu_{2}\right)$.

In general, the duality defines the adjoint kernel operator beyond of a set of functions, that is for functionals that not necessarily have an integral representation. In [52, Prop. 2.1] there is an attempt to save this handicap. However, if we restrict our attention to integrable functions we save this problem. For the aim of simplicity we assume that functionals have an integral representation, or in other words we will work with the Köthe adjoint operator defined on the Köthe dual space. For all $f \in X\left(\mu_{2}\right)$ and for all $g \in\left(Y\left(\mu_{1}\right)\right)^{\prime}$, we know that $\left\langle T_{K} f, g\right\rangle=\left\langle f, T_{K}^{\prime} g\right\rangle$, where $T_{K}^{\prime}:\left(Y\left(\mu_{1}\right)\right)^{\prime} \rightarrow\left(X\left(\mu_{2}\right)\right)^{\prime}$. Applying Fubini's Theorem we obtain that

$$
\left(T_{K}^{\prime} g\right)\left(\omega_{2}\right)=\int_{\Omega_{1}} K\left(\omega_{1}, \omega_{2}\right) g\left(\omega_{1}\right) d \mu_{1}\left(\omega_{1}\right), \quad g \in\left(Y\left(\mu_{1}\right)\right)^{\prime} .
$$

Therefore it is sufficient that $K\left(\cdot, \omega_{2}\right)$ be $\mu_{2}$-integrable for almost every $\omega_{2} \in \Omega_{2}$. Let us define $K^{\prime}(x, y):=K(y, x)$, which is the kernel function for the adjoint operator. So, Fubini's Theorem implies that $K^{\prime} \in L^{1}\left(\mu_{2} \otimes \mu_{1}\right)$, hence the requirement $K \in L^{1}\left(\mu_{1} \otimes \mu_{2}\right)$ makes sense. 
In fact this assumption implies that both $T_{K}$ and $T_{K}^{\prime}$ are Carleman operators (see e.g. [94] for the definition of this type of operators). The theory of kernel operators is widely studied, here we refer the reader to $[\mathbf{1}$, Ch. 5], [46], [60, §15 and §16], [73, Sect. 3.3], [76], [108, Ch. 13] and the references therein.

In the present memoir we work with Banach function spaces $X$ and $Y$, which are based on the same measure space $(\Omega, \Sigma, \mu)$ or in Banach function spaces based on the Lebesgue measure. Also, in general, the kernel functions that we consider are positive. So in each case we will adapt the notation above to the context.

Often, we will need to compute the double norm of $K$ or of $K^{\prime}$. In order to decide, in the usual situation, over which of the two variables we compute first the norm, let us introduce the following notation. Let $(\Omega, \Sigma, \mu)$ be a measure space and let $K \in L^{1}(\mu \otimes \mu)$ be a positive kernel. Let $\omega_{2} \in \Omega$ and let $Z$ be a quasi-Banach function space, we denote by $\|K\|_{Z, \omega_{1}}$ the norm in $Z$ of the function $K\left(\cdot, \omega_{2}\right) \in Z$, i.e. $\left(\|K\|_{Z, \omega_{1}}\right)\left(\omega_{2}\right):=\left\|K\left(\cdot, \omega_{2}\right)\right\|_{Z}$, where $\omega_{2} \in \Omega$. By $\|K\|_{Z, \omega_{2}}$ we denote the norm for the second coordinate, i.e. $\left(\|K\|_{Z, \omega_{2}}\right)\left(\omega_{1}\right):=$ $\left\|K\left(\omega_{1}, \cdot\right)\right\|_{Z}$, where $\omega_{1} \in \Omega$. Again it is assumed that $K\left(\omega_{1}, \cdot\right) \in Z$. Thus, if $Y$ is a quasiBanach function space, in what follows the notations that we use for the double norms are \|\|$K\left\|_{Z, \omega_{2}}\right\|_{Y}$ and \|\|$K\left\|_{Z, \omega_{1}}\right\|_{Y}$. Observe that \|\|$K\left\|_{Z, \omega_{2}}\right\|_{Y}=\|\| K^{\prime}\left\|_{Z, \omega_{1}}\right\|_{Y}$ and also that \|\|$K\left\|_{Z, \omega_{1}}\right\|_{Y}=\|\| K^{\prime}\left\|_{Z, \omega_{2}}\right\|_{Y}$.

For the aim of simplicity, when $Z=L^{q}$ we will write $\|K\|_{q, \omega_{2}}$ and $\|K\|_{q, \omega_{1}}$. For example

$$
\|\| K\left\|_{q, \omega_{2}}\right\|_{p}=\left(\int_{\Omega}\left(\int_{\Omega}\left|K\left(\omega_{1}, \omega_{2}\right)\right|^{q} d \mu\left(\omega_{2}\right)\right)^{p / q} d \mu\left(\omega_{1}\right)\right)^{1 / p} .
$$

The compactness property has an important role in the theory of kernel operators. In [94, Th. 2.3] we can find equivalent conditions for a kernel operator to be a Hille-Tamarkin operator, that is a kernel operator $T_{K}: X \rightarrow Y$ such that \|\|$K\left\|_{X^{\prime}, \omega_{2}}\right\|_{Y}<\infty$.

We also will use the Minkowski's integral inequality. Let $0<p \leq q<\infty$. Let $\left(\Omega_{1}, \Sigma_{1} \mu_{1}\right)$ and $\left(\Omega_{2}, \Sigma_{2}, \mu_{2}\right)$ be two measure spaces and let $F: \Omega_{1} \times \Omega_{2} \rightarrow \mathbb{R}$ be a $\Sigma_{1} \otimes \Sigma_{2}$-measurable function. Then

$$
\begin{aligned}
\left(\int_{\Omega_{2}}\left(\int_{\Omega_{1}}\left|F\left(\omega_{1}, \omega_{2}\right)\right|^{p} d \mu_{1}\left(\omega_{1}\right)\right)^{q / p}\right. & \left.d \mu_{2}\left(\omega_{2}\right)\right)^{1 / q} \\
\leq & \leq\left(\int_{\Omega_{1}}\left(\int_{\Omega_{2}}\left|F\left(\omega_{1}, \omega_{2}\right)\right|^{q} d \mu_{2}\left(\omega_{2}\right)\right)^{p / q} d \mu_{1}\left(\omega_{1}\right)\right)^{1 / p} .
\end{aligned}
$$

If $F$ is a kernel function, so is $F^{\prime}$, thus by using the notation with norms given above we have

$$
\|\| F\left\|_{p, \omega_{1}}\right\|_{q} \leq\|\| F\left\|_{q, \omega_{2}}\right\|_{p} \Longleftrightarrow\|\| F^{\prime}\left\|_{p, \omega_{2}}\right\|_{q} \leq\|\| F^{\prime}\left\|_{q, \omega_{1}}\right\|_{p} .
$$

See [49] for more information or [95, Th. 2.3] for a generalization.

Finally, we refer to [110] for the standard formulas that we use in some examples all along this memoir. 
CHAPTER 2

\section{GENERALIZED KÖTHE DUAL SPACE}

In this chapter we study the representation for the Köthe dual space of the $p$-th power of a Banach function space. To do this we introduce the definition of the Köthe $p$-dual space, which will play a central role in the following chapters. We also define the Köthe $p$ adjoint operator, which is always $p$-th power factorable. Then we study the properties of these two concepts and provide some applications as a characterization of Hilbert spaces or a factorization through Lorentz spaces.

\subsection{The Köthe $p$-dual space}

We start by showing that for general Banach function spaces over a finite non atomic measure and $p \in(0, \infty) \backslash\{1\}$, we have the following inequality

$$
\left(X^{\prime}\right)_{[p]} \neq\left(X_{[p]}\right)^{\prime} .
$$

Let $X$ be a Banach function space over a non atomic finite measure. Assume that $\left(X^{\prime}\right)_{[p]}=$ $\left(X_{[p]}\right)^{\prime}$, then if $1<p<\infty$ we have $X^{\prime} \subseteq\left(X^{\prime}\right)_{[p]}=\left(X_{[p]}\right)^{\prime} \subseteq X^{\prime}$ and if $0<p<1$ we have $X^{\prime} \subseteq\left(X_{[p]}\right)^{\prime}=\left(X^{\prime}\right)_{[p]} \subseteq X^{\prime}$. Thanks to [79, Prop. 2.26], $X^{\prime}=\left(X^{\prime}\right)_{[p]}=L^{\infty}$, thus $X=L^{1}$. For $p>1$ we have that $X^{\prime}=\left(X_{[p]}\right)^{\prime}=\left(L^{1 / p}\right)^{\prime}=\{0\}$ (see [107, Ch. 15]), which is a contradiction. If $p<1$ we have that

$$
L^{\infty}=L_{[p]}^{\infty}=\left(X^{\prime}\right)_{[p]}=\left(X_{[p]}\right)^{\prime}=L^{(1 / p)^{\prime}}=L^{1 /(1-p)}
$$

then $p=1$, which is a contradiction.

Nevertheless, we have that $\left(X_{[p]}\right)^{\prime}=\left(X^{L^{p}}\right)_{[p]}$ for every $p \in(0, \infty)$ (Proposition 1.5(11)). This motivates the introduction of the space $X^{p}$ that plays the role of the Köthe dual but satisfying this equality.

Definition 2.1. Let $0<p \leq \infty$. Let $X$ be a quasi-Banach function space, we define its Köthe $p$-dual space $X^{p}$ as

$$
X^{p}:=\left\{g \in L^{0}: g f \in L^{p} \text { for every } f \in X\right\}=X^{L^{p}} .
$$

Notice that $X^{p}$ may be trivial, for example if $X:=L^{1}[0,1]$ we have that $X^{p}=\{0\}$ for every $1<p<\infty$. We will see that this space is useful for representing the Köthe dual space $\left(X_{[p]}\right)^{\prime}$. In what follows we set some properties of this space in terms of the space $X^{p}$. 
The papers of reference are [71] and [12]. In the following lemma we reproduce some of these results in the context of finite measure. Observe also that $X^{p}$ has weak order unit, whenever $X^{p} \subseteq L^{p}$ and [12, Rem. 2.3] (by the below Lemma 2.5(1) this is always true in our case), but in general we cannot affirm that $X^{p}$ has weak order unit, it depends on the election of $X$.

2.1.1. Computation rules for the Köthe $p$-dual space. In [12],[59, Sect. 2] and [71, Sect. 2, Cor. 1] we can find several properties involving general spaces of multipliers. Let us now prove some properties for our setting of Köthe $p$-dual and $p$-th power spaces. Recall that all spaces are based on the same finite measure $\mu$.

Lemma 2.2. Let $X$ and $Y$ be Banach function spaces over the same finite measure. The following statements hold:

(1) If $0<p \leq q \leq \infty$, then $X^{q} \subseteq X^{p}$.

(2) If $1 \leq p<\infty$, then $X^{p} \subseteq\left(X^{p}\right)_{[p]} \subseteq X^{\prime}$.

(3) If $0 \leq p \leq q<\infty$, then $\left(X_{[p]}\right)^{q}=\left(X^{p q}\right)_{[p]}$ and $\left(X^{q}\right)_{[p]}=\left(X_{[p]}\right)^{q / p}$.

(4) If $0<p \leq \infty$ and $X \subseteq Y$ then $Y^{p} \subseteq X^{p}$.

(5) If $0<p \leq q \leq \infty$, then $\left(X^{p}\right)^{q} \subseteq\left(X^{q}\right)^{p}$.

(6) If $1 \leq p \leq q \leq \infty$, then $\left(X_{[p]}\right)^{q} \subseteq X^{p}$.

(7) If $s \leq r \leq t$ and $1 \leq p \leq q \leq \infty$, then

$$
\left(X_{[q]}\right)^{t} \subseteq\left(X_{[q]}\right)^{r} \subseteq\left(X_{[p]}\right)^{r} \subseteq\left(X_{[p]}\right)^{s} .
$$

(8) If $r q \leq t p$ and $1 \leq p \leq q \leq \infty$, then $\left(X_{[p]}\right)^{t} \subseteq\left(X_{[q]}\right)^{r}$.

Proof. (1) Let $f \in X^{q}$. Then $L^{q} \subseteq L^{p}$ implies that for some $C>0$

$$
\|f\|_{X^{p}}=\sup _{g \in B_{X}}\|f g\|_{p} \leq C \sup _{g \in B_{X}}\|f g\|_{q}=C\|f\|_{X^{q}} .
$$

(2) By Proposition 1.5(6), $X^{p} \subseteq\left(X^{p}\right)_{[p]}$ and $X \subseteq X_{[p]}$. Now, by Proposition 1.5(11), we have that $X^{p} \subseteq\left(X^{p}\right)_{[p]}=\left(X_{[p]}\right)^{\prime} \subseteq X^{\prime}$.

(3) It follows from the definitions and the properties (1) and (10) of Proposition 1.5

$$
\left(X_{[p]}\right)^{q}=\left(X_{[p]}\right)^{L^{q}}=\left(X_{[p]}\right)^{L_{[1 / q]}^{1}}=\left(X_{[p]}\right)^{L_{[p / p q]}^{1}}=\left(X_{[p]}\right)^{\left(L_{[1 / p q]}^{1}\right)_{[p]}}=\left(X_{[p]}\right)^{L_{[p]}^{p q}}=\left(X^{p q}\right)_{[p]} .
$$

Thus $\left(X^{q}\right)_{[p]}=\left(X^{p(q / p)}\right)_{[p]}=\left(X_{[p]}\right)^{q / p}$.

(4) Since $X \subseteq Y,\|f\|_{X^{p}}=\sup _{g \in B_{X}}\|f g\|_{p} \leq C \sup _{g \in B_{Y}}\|f g\|_{p}=C\|f\|_{Y^{q}}$, for some $C>0$, which implies the statement.

(5) Applying twice (4) and once (1), since $X^{q} \subseteq X^{p}$, we have $\left(X^{p}\right)^{q} \subseteq\left(X^{p}\right)^{p} \subseteq\left(X^{q}\right)^{p}$.

(6) Since $1 \leq p \leq q, L^{q} \subseteq L^{p}$ and by Proposition 1.5(6) $X \subseteq X_{[p]}$. Let $f \in\left(X_{[p]}\right)^{q}$. Then $f g \in L^{q} \subseteq L^{p}$ for all $g \in X_{[p]}$, in particular for all $g \in X$ and so $f \in X^{p}$. 
(7) Since $1 \leq p \leq q$, it holds $X_{[p]} \subseteq X_{[q]}$ and $X^{q} \subseteq X^{p}$. Thus, if $s \leq r \leq t$,

$$
\left(X_{[q]}\right)^{t} \subseteq\left(X_{[q]}\right)^{r} \subseteq\left(X_{[p]}\right)^{r} \subseteq\left(X_{[p]}\right)^{s} .
$$

(8) Using the given conditions: $\left(X_{[p]}\right)^{t}=\left(X^{p t}\right)_{[p]} \subseteq\left(X^{q r}\right)_{[p]} \subseteq\left(X^{q r}\right)_{[q]}=\left(X_{[q]}\right)^{r}$.

Example 2.3. Let $L^{p}(m)$ denote, for $1 \leq p<\infty$, the space of $p$-integrable real-valued functions with respect to the vector measure $m$. Assume that the Banach function space $L^{1}(m)$ has the Fatou property (see [19, Prop. 3.38] for equivalences with this requirement). By Lemma 2.2(3) we have

$$
L^{p}(m)=\left(L^{1}(m)\right)_{[1 / p]}=\left(\left(L^{1}(m)\right)^{\prime \prime}\right)_{[1 / p]}=\left(\left(\left(L^{1}(m)\right)^{\prime}\right)_{[1 / p]}\right)^{p}=\left(\left(L^{p}(m)\right)^{p}\right)^{p}
$$

Applying [12, Prop. 5.3] we obtain that $L^{p}(m)$ is Fatou and $p$-convex with constant one. These facts can be verified directly.

Let us now deduce some other consequences.

Proposition 2.4. Let $X$ and $Y$ be Banach function spaces over the same finite measure. The following statements hold:

(1) Let $0<p, q<\infty$, then $X^{p}=\left(\left(X_{[1 / q]}\right)^{p q}\right)_{[q]}$. In particular $X^{\prime}=\left(\left(X_{[1 / q]}\right)^{q}\right)_{[q]}$.

(2) Let $1 \leq p<\infty$, then $X^{p} \subseteq\left(X_{[p]}\right)^{\prime} \subseteq X^{\prime}$.

(3) Let $0<p<\infty$, then $Y \subseteq X^{p}$ if and only if $X \subseteq Y^{p}$.

Proof. (1) We proceed directly using the definition of Köthe $p$-dual. $L^{p}=\left(L^{1}\right)_{[1 / p]}$, then we apply the properties (1) and (11) of Proposition 1.5

$$
X^{p}=\left(\left(X^{p}\right)_{[1 / q]}\right)_{[q]}=\left(\left(X_{[1 / q]}\right)^{\left(L^{p}\right)_{[1 / q]}}\right)_{[q]}=\left(\left(X_{[1 / q]}\right)^{\left(\left(L^{1}\right)_{[1 / p]}\right)_{[1 / q]}}\right)_{[q]}=\left(\left(X_{[1 / q]}\right)^{p q}\right)_{[q]} .
$$

(2) Apply Proposition 1.5(6) and Lemma 2.2(4). It also can be proved directly with Lemma 2.2(2).

(3) Assume that $Y \subseteq X^{p}$. By this hypothesis and Proposition 1.5(11) we have that $Y_{[p]} \subseteq$ $\left(X^{p}\right)_{[p]}=\left(X_{[p]}\right)^{\prime}$, hence $X_{[p]} \subseteq\left(X_{[p]}\right)^{\prime \prime} \subseteq\left(Y_{[p]}\right)^{\prime}$. Let $g \in X$. Then, for some $C>0$, it holds

$$
\|g\|_{Y^{p}}=\left\||g|^{p}\right\|_{\left(Y^{p}\right)_{[p]}}^{1 / p}=\left\||g|^{p}\right\|_{\left(Y_{[p]}\right)^{\prime}}^{1 / p} \leq C\left\||g|^{p_{\|^{\prime}}^{1 / p}}=C\right\| g \|_{X} .
$$

The converse is analogous, just changing the roles of $X$ and $Y$.

The following lemma is the best approximation to the Köthe $p$-dual space that we have reached in terms of $L^{p}$-spaces.

Lemma 2.5. Let $1 \leq p<\infty$. Let $X$ be a Banach function space over a positive finite measure. Then

(1) $X^{p} \subseteq L^{p}$.

(2) Let $q \leq r, p \leq \infty$ be such that $\frac{1}{q}=\frac{1}{p}+\frac{1}{r}$. If $X \subseteq L^{r}$, then $L^{p} \subseteq X^{q}$. 
Proof. Let us prove (1). In order to obtain a bound for the norm of the inclusion map, let us adapt the proof of Hölder-Rogers inequality as given in [8, Ch. 1 Th. 2.4]. Let be $f \in X^{p}$, since $\chi_{\Omega} \in X$ then $f=f \chi_{\Omega} \in L^{p}$. In order to prove the continuity we consider the following

$$
\begin{aligned}
\|f\|_{p}=\left\|f \chi_{\Omega}\right\|_{p} & =\left\|\chi_{\Omega}\right\|_{X}\left\|f \frac{\chi_{\Omega}}{\left\|\chi_{\Omega}\right\|_{X}}\right\|_{p} \leq\left\|\chi_{\Omega}\right\|_{X} \sup _{g \in X \backslash\{0\}}\left\|f \frac{g}{\|g\|_{X}}\right\|_{p} \\
& =\left\|\chi_{\Omega}\right\|_{X} \sup _{g \in B_{X}}\|f g\|_{p}=\left\|\chi_{\Omega}\right\|_{X}\|f\|_{X^{p}} .
\end{aligned}
$$

So $X^{p} \subseteq L^{p}$. For the assertion (2), we use Hölder-Rogers inequality

$$
\|f\|_{X^{q}}=\sup _{g \in B_{X}}\|g f\|_{q} \leq \sup _{g \in B_{X}}\|g\|_{r}\|f\|_{p}=\sup _{g \in B_{X}}\left\|g \chi_{\Omega}\right\|_{r}\|f\|_{p}=\left\|\chi_{\Omega}\right\|_{X^{r}}\|f\|_{p},
$$

hence $L^{p} \subseteq X^{q}$, which completes the proof.

As we have seen, the Köthe $p$-dual space of a Banach function space is quite similar to the $L^{p}$ space.

Corollary 2.6. Let $X$ be a Banach function space over a finite measure. Let $1 \leq p<\infty$. Then $X^{p}=L^{p}$ if and only if $X=L^{\infty}$.

Proof. We only need to show that $X^{p}=L^{p}$ implies that $X=L^{\infty}$. It follows from Proposition 2.4(3), since $L^{p} \subseteq X^{p}$ implies that $X \subseteq\left(L^{p}\right)^{p}=L^{\infty}$. Then $X=L^{\infty}$.

2.1.2. The Köthe $p$-adjoint operator. Let us now study operators defined on Köthe $p$-dual spaces. They provide a natural $p$-th power factorization, for $1 \leq p<\infty$, as the next diagram shows

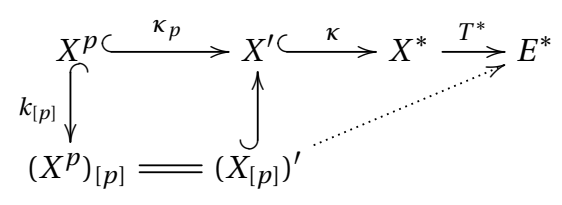

DiAgram 2.1. Köthe $p$-adjoint operator

Definition 2.7. Let $1 \leq p<\infty$. Let $X$ be a quasi-Banach function space, $E$ a Banach space and $T: E \rightarrow X$ an operator. It is defined the operator $T^{p}: X^{p} \rightarrow E^{*}$ as $T^{p}:=\left.T^{*}\right|_{X^{p}}$, that we will call Köthe $p$-adjoint operator. For $p=1$, this operator coincides with the Köthe adjoint operator $T^{\prime}$.

The following proposition gives examples of $p$-th power factorable operators. We also provide an expression for the extension map, which will be useful in the sequel.

Proposition 2.8. Let $1 \leq p<\infty$. Let $E$ be a Banach space, and let $X$ be an order continuous Banach function space over a finite measure such that $X^{p}$ is order continuous. Let $T: E \rightarrow X$ 
be an operator. Then the Köthe $p$-adjoint operator $T^{p}$ is $p$-th power factorable, and the extension operator is $\left(T^{p}\right)_{[p]}:=\left(i_{[p]} \circ T\right)^{*}=T^{*} \circ\left(i_{[p]}\right)^{\prime}$.

Proof. The first assertion is immediate from Diagram 2.1. Let us formalize this fact. Let $f \in X^{p} \subseteq X^{\prime}=X^{*}$, and $T^{p}=\left.T^{*}\right|_{X^{p}}$, hence

$$
\left\|T^{p}(f)\right\|_{E^{*}}=\left\|T^{*}(f)\right\|_{E^{*}}=\sup _{g \in B_{E}}\left|\left\langle g, T^{*}(f)\right\rangle\right|=\sup _{g \in B_{E}}|\langle T(g), f\rangle| .
$$

Since $\mu$ is finite, $X \subseteq X_{[p]}$, and by Proposition 1.5(11), $\left(X_{[p]}\right)^{\prime}=\left(X^{p}\right)_{[p]}$, thus

$$
\begin{aligned}
\sup _{g \in B_{E}}|\langle T(g), f\rangle| & \leq\|T\| \sup _{h \in B_{X}}|\langle h, f\rangle| \leq C\|T\| \sup _{h \in B_{X}}|\langle h, f\rangle| \\
& \leq C\|T\|\|f\|_{\left(X_{[p]}\right)^{\prime}}=C\|T\|\|f\|_{\left(X^{p}\right)_{[p]}},
\end{aligned}
$$

for some $C>0$. Since $X^{p}$ is order continuous by hypothesis, we apply Proposition 1.8 (picking $X^{p}$ instead of $X$ ) and we obtain that $T^{p}: X^{p} \rightarrow E^{*}$ is $p$-th power factorable.

For the second assertion, by definition of Köthe $p$-adjoint we have $T^{p}=\left(T^{p}\right)_{[p]} \circ k_{[p]}$, where $k_{[p]}: X^{p} \hookrightarrow\left(X^{p}\right)_{[p]}$ is the canonic inclusion and $\left(T^{p}\right)_{[p]}$ is the unique extension. On the other hand $i_{[p]}: X \hookrightarrow X_{[p]}$ is canonic and so is $\left(i_{[p]}\right)^{*}:\left(X_{[p]}\right)^{*}=\left(X_{[p]}\right)^{\prime}=\left(X^{p}\right)_{[p]} \hookrightarrow X^{*}$. Since $X$ is order continuous by Proposition 1.5(5), so is $X_{[p]}$. Then, by uniqueness of the extension, we have that $\left(T^{p}\right)_{[p]}:\left(X_{[p]}\right)^{\prime} \stackrel{(i[p])^{\prime}}{\longrightarrow} X^{*} \stackrel{T^{*}}{\rightarrow} E^{*}$.

Remark 2.9. Let us analyze the factorization of $T^{2}: X^{2} \rightarrow E^{*}$. Under the requirements of Proposition 2.8 we have that $T^{2}=\left(T^{2}\right)_{[2]} \circ i_{[2]}$. On the other hand by [97, Prop. 3.1] $X^{2}$ is 2convex. If $X$ is 4-convex, then $X_{[2]}$ is 2-convex, and so $\left(X^{2}\right)_{[2]}=\left(X_{[2]}\right)^{\prime}$ is 2-concave (see e.g. [23, Lem. 2]). Therefore $i_{[2]}: X^{2} \rightarrow L^{2} \rightarrow\left(X^{2}\right)_{[2]}$ (see [66, Cor. 1.f.15(iii)]). In consequence $T^{2}$ factors through a Hilbert space.

From [71, Th. 4] and [12, Rem. 6.8] we deduce the following example (see also [68]).

Example 2.10. See $[\mathbf{6 0}, \mathbf{9 0}]$ for theory and applications of Orlicz spaces. Let $(\mathbb{R}, \Sigma, \mu)$ be a finite measure space. Let $\varphi$ be an Orlicz function, i.e. a convex, continuous, increasing and unbounded function defined on $[0, \infty)$, so that $\varphi(0)=0$. The Orliz space is defined by

$$
L^{\varphi}:=\left\{f \in L^{0}: \inf \left\{\lambda>0: \int_{\mathbb{R}} \varphi(|f(\omega)| / \lambda) d \mu(\omega) \leq 1\right\}<\infty\right\},
$$

where $\varphi$ is an Orlicz function that satisfies the $\Delta_{2}$-condition, i.e. there exists $k>0$ and $u_{0} \geq 0$ such that $\varphi(2 u) \leq k \varphi(u)$ for every $u \geq u_{0}$. Assume that $\frac{\varphi(u)}{u}$ is increasing and

$$
\lim _{u \rightarrow \infty} \sup \frac{u}{\varphi(u)}=\lim _{u \rightarrow 0} \sup \frac{\varphi(u)}{u}=0 .
$$

Let us define $\varphi_{0}(u):=\sup _{\nu>0}\{2 u \nu-\varphi(v)\}$ for $u \geq 0$. Then $\left(L^{\varphi}\right)^{\prime}=L^{\varphi_{0}}$ ([12, Rem. 6.8]). 
Let us consider the 4 -convex space $X_{\varphi}:=\left(\left(L^{\varphi}\right)^{4}\right)^{4}$. The one-dimensional Riesz transform $R: L^{\varphi} \rightarrow L^{\varphi}$, defined by

$$
(R f)(x):=c \int_{\mathbb{R}} \frac{x-y}{|x-y|} f(y) d \mu(y),
$$

where $c:=\frac{\Gamma(1 / 2)}{\pi^{1 / 2}}$, is continuous (see [58, Th. 3.11]). Let $i: L^{\varphi} \hookrightarrow X_{\varphi}$ be the inclusion map, thus we can define $T:=i \circ R: L^{\varphi} \rightarrow X_{\varphi}$, which is a Riesz transform. In this case the operator $T$ has Köthe 2-adjoint operator $T^{2}:\left(X_{\varphi}\right)^{2} \rightarrow L^{\varphi_{0}}$, and $T^{2}$ is still the Riesz transform. In virtue of the remark above, it factors through a Hilbert space.

Remark 2.11. In order to summarize the relations and spaces that we have considered in this chapter we present the following diagram, for $1 \leq q \leq p<\infty$ and $u, v>1$. $X$ is a Banach function space over a finite measure, $E$ is a Banach space and $T: E \rightarrow X$ is an operator.

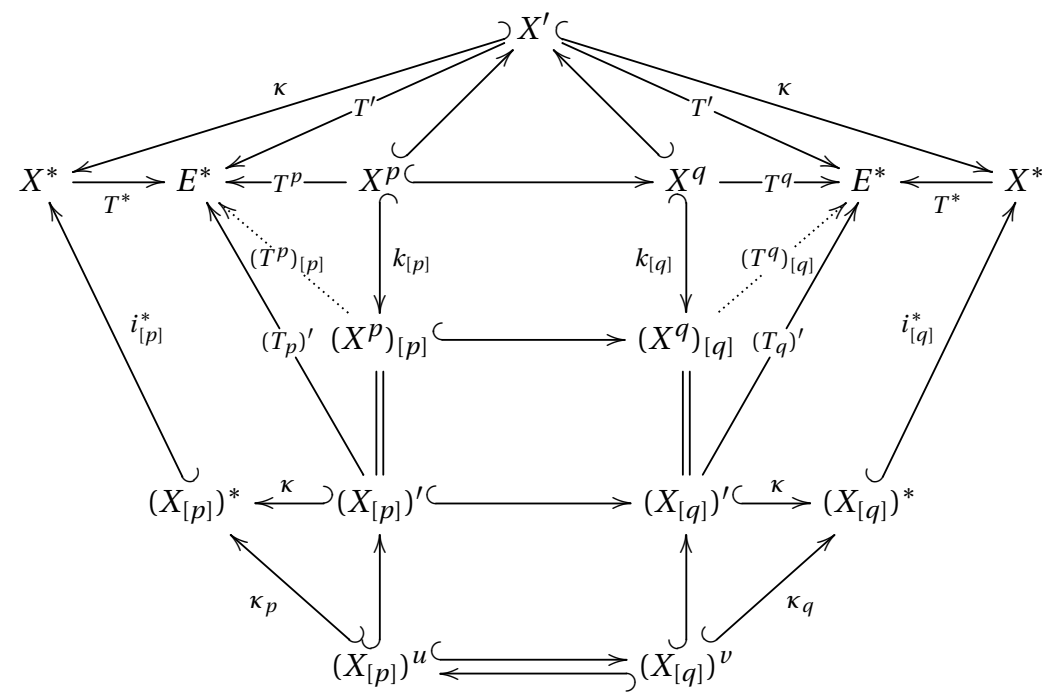

Diagram 2.2. Context diagram for the Köthe $p$-dual space and Köthe $p$-adjoint operator

\subsection{Some geometrical aspects}

The geometric structure of the space of multipliers is strongly connected with the notions of concavity and convexity (see e.g. [66, Sect. 1.c, 1.d and 1.e] or in a more general context [35, Ch. 9, 10 and 11]). Now we are going to study the $p$-convexity and $q$-concavity, type and coptype of the Köthe $r$-dual spaces. In general for a given Banach space $E$, these notions describe the local behavior (understanding local as finite-dimensional) of the spaces $\ell^{p}\left(E_{n}\right)$, where $E_{n}$ is an $n$-dimensional linear subspace of $E$. 
Let us recall Kwapień's characterization of Hilbert spaces [64], $X$ is isomorphic to a Hilbert space if and only if has type 2 and cotype 2. See [88, Ch. 3] for more details and applications.

2.2.1. Convexity and concavity. Let us state a first corollary.

Corollary 2.12. Let $1 \leq p<\infty$. Let $E$ be a $p^{\prime}$-convex Banach lattice and $X$ an order continuous Banach function space. Let $T: E \rightarrow X$ be an operator. If $T^{p}: X^{p} \rightarrow E^{*}$ is positive, then $T^{p}$ factors through $L^{p}$.

Proof. Since $E$ is $p^{\prime}$-convex, $E^{*}$ is $p$-concave ([66, Prop. 1.d.4(iii)]), then $T^{p}$ is $p$ concave too, since $T^{p}$ is positive ([66, Prop. 1.d.9]). On the other hand thanks to [12, Lem. 5.1] we have that $X^{p}$ is $p$-convex, thus Maurey-Rosenthal's Theorem 1.4 ensure us that $T^{p}$ factors through $L^{p}$.

In particular for $p=1$ we have the following. If $T: E=L^{\infty} \rightarrow X, T^{\prime}$ is positive and $T^{\prime}\left(X^{\prime}\right) \subseteq L^{1}$, then $T^{\prime}$ factors through $L^{p}$ for every $1 \leq p<\infty$.

Let $p \in[1, \infty]$, it is well known that $X^{p}$ is $p$-convex with constant 1. (see e.g. [97, Prop 3.1]). However, this result does not hold for the $p$-concavity. For instance $\left(L^{p}\right)^{p}=L^{\infty}$, which is not $p$-concave for any $p<\infty$. The following theorem sheds some light on the $p$ concave case. In fact it is a generalization of [66, Prop. 1.d.4](iii), see also [62]. The proof is adapted from [83, Lem. 2.2] (see also [41, Lem. 4.2]).

Theorem 2.13. Let $1 \leq r \leq p \leq \infty$ and let $X$ and $Y$ be two Banach function spaces over the same measure, such that $Y$ is $r$-concave and $X$ is $p$-convex. Then $X^{Y}$ is $q$-concave for $\frac{1}{q}=\frac{1}{r}-\frac{1}{p}$.

Proof. We assume without loss of generality that the involved concavity and convexity constants are equal to 1 . Let us take $n \in \mathbb{N}, f_{1}, \ldots, f_{n} \in X^{Y}$ and $g_{1}, \ldots, g_{n} \in B_{X}$. Thanks to [71, Prop. 3] it is clear that $\ell^{q}=\left(\ell^{p}\right)^{r}$, so for an element $\left(\tau_{i}\right)_{i} \in \ell^{q}$

$$
\left\|\left(\tau_{i}\right)_{i}\right\|_{q}=\left(\sum_{i}\left|\tau_{i}\right|^{q}\right)^{1 / q}=\sup _{\left(\lambda_{i}\right)_{i} \in B_{\ell} p}\left(\sum_{i}\left|\lambda_{i} \tau_{i}\right|^{r}\right)^{1 / r}
$$

Since $X$ is $p$-convex with constant 1 , if $\left(\lambda_{i}\right)_{i} \in B_{\ell^{p}}$, (note that $\lambda_{i} g_{i} \in X$ ) we have

$$
\left\|\left(\sum_{i=1}^{n}\left|\lambda_{i} g_{i}\right|^{p}\right)^{1 / p}\right\|_{X} \leq\left(\sum_{i=1}^{n}\left\|\lambda_{i} g_{i}\right\|_{X}^{p}\right)^{1 / p}=\left(\sum_{i=1}^{n}\left|\lambda_{i}\right|^{p}\left\|g_{i}\right\|_{X}^{p}\right)^{1 / p} \leq\left(\sum_{i=1}^{n}\left|\lambda_{i}\right|^{p}\right)^{1 / p} \leq 1,
$$


hence $\left(\sum_{i=1}^{n}\left|\lambda_{i} g_{i}\right|^{p}\right)^{1 / p} \in B_{X}$. So, applying (2.1), that $Y$ is $r$-concave and (2.2), we obtain

$$
\begin{aligned}
\left(\sum_{i=1}^{n}\left\|f_{i} g_{i}\right\|_{Y}^{q}\right)^{1 / q} & =\sup _{\left(\lambda_{i}\right)_{i} \in B_{\ell} p}\left(\sum_{i=1}^{n}\left|\lambda_{i}\right|^{r}\left\|f_{i} g_{i}\right\|_{Y}^{r}\right)^{1 / r}=\sup _{\left(\lambda_{i}\right)_{i} \in B_{\ell} p}\left(\sum_{i=1}^{n}\left\|\lambda_{i} g_{i} f_{i}\right\|_{Y}^{r}\right)^{1 / r} \\
& \leq \sup _{\left(\lambda_{i}\right)_{i} \in B_{\ell} p}\left\|\left(\sum_{i=1}^{n}\left|\lambda_{i} g_{i} f_{i}\right|^{r}\right)^{1 / r}\right\|_{Y}=\sup _{\left(\lambda_{i}\right)_{i} \in B_{\ell} p}\left\|\left(\sum_{i=1}^{n}\left|\lambda_{i} g_{i}\right|^{p}\right)^{1 / p}\left(\sum_{i=1}^{n}\left|f_{i}\right|^{q}\right)^{1 / q}\right\|_{Y} \\
& \leq \sup _{g \in B_{X}}\left\|g\left(\sum_{i=1}^{n}\left|f_{i}\right|^{q}\right)^{1 / q}\right\|_{Y}=\left\|\left(\sum_{i=1}^{n}\left|f_{i}\right|^{q}\right)^{1 / q}\right\|_{X^{Y}} .
\end{aligned}
$$

Given $\varepsilon>0$ we can select $\left\{g_{1}, \ldots, g_{n}\right\} \in B_{X}$ such that $\left\|f_{i}\right\|_{X^{Y}} \leq\left\|f_{i} g_{i}\right\|_{Y}+\varepsilon /\left(n^{1 / q}\right)$ for each $i=1, \ldots, n$. Then applying $(2.3)$

$$
\begin{aligned}
\left(\sum_{i=1}^{n}\left\|f_{i}\right\|_{X^{Y}}^{q}\right)^{1 / q} & \leq\left(\sum_{i=1}^{n}\left(\left\|f_{i} g_{i}\right\|_{Y}+\frac{\varepsilon}{n^{1 / q}}\right)^{q}\right)^{1 / q} \leq\left(\sum_{i=1}^{n}\left(\left\|f_{i} g_{i}\right\|_{Y}\right)^{q}\right)^{1 / q}+\left(\sum_{i=1}^{n}\left(\frac{\varepsilon}{n^{1 / q}}\right)^{q}\right)^{1 / q} \\
& =\left(\sum_{i=1}^{n}\left(\left\|f_{i} g_{i}\right\|_{Y}\right)^{q}\right)^{1 / q}+\varepsilon \leq\left\|\left(\sum_{i=1}^{n}\left|f_{i}\right|^{q}\right)^{1 / q}\right\|_{X^{Y}}+\varepsilon
\end{aligned}
$$

for every $\varepsilon>0$, which yields us that $X^{Y}$ is $q$-concave.

The conclusion of this theorem may fail without the requirement on the $p$-convexity of $X$.

Example 2.14. Let $1 \leq r<p<\infty$ and $r \leq q<\infty$ be such that $\frac{1}{r}=\frac{1}{p}+\frac{1}{q}$. Let us take $X:=L^{s}$ for $r \leq s<p$. Then $X$ is not $p$-convex and $X^{r}=L^{t}$, where $\frac{1}{t}=\frac{1}{r}-\frac{1}{s}$. Taking $Y:=L^{r}$ in the previous theorem and taking into account that $\frac{1}{t}=\frac{1}{r}-\frac{1}{s}=\frac{1}{q}+\frac{1}{p}-\frac{1}{s}$, we have that $\frac{1}{t}-\frac{1}{q}=\frac{1}{p}-\frac{1}{s}<0$ since $s<p$, therefore $q<t$, and so $X^{r}=L^{t}$ cannot be $q$-concave.

Remark 2.15. For the case of Lebesgue spaces we have $\left(L^{q}\right)^{p}=\{0\}$ for $1 \leq q<p \leq \infty$. However, it is not in general true that $\left(X^{q}\right)^{p}=\{0\}$ for every $X$ and every $1 \leq q<p \leq \infty$. Take $1 \leq q<r$ and choose $s \geq 1$ so that $\frac{1}{s}=\frac{1}{q}-\frac{1}{r}$. Then, for $q<p<s$ and $X:=L^{r}$, we conclude that $X^{q}=L^{s}$ and so $\left(X^{q}\right)^{p}=L^{t} \neq\{0\}$, where $\frac{1}{t}=\frac{1}{p}-\frac{1}{s}$.

The following corollary provides conditions on the indexes $p$ and $q$ that implies $\left(X^{q}\right)^{p}=$ $\{0\}$ when $q<p$.

Corollary 2.16. Let $1 \leq q<p \leq \infty$ and let $r>1$ such that $\frac{1}{r}<\frac{1}{q}-\frac{1}{p}$. Let $X$ be an $r$-convex Banach function space over a non-atomic finite measure. Then $\left(X^{q}\right)^{p}=\{0\}$.

Proof. By the previous theorem $X^{q}$ is $s$-concave for $s \geq 1$ such that $\frac{1}{q}=\frac{1}{r}+\frac{1}{s}$. Since $\frac{1}{p}<\frac{1}{q}-\frac{1}{r}=\frac{1}{s}$, we have that $s<p$. Now, since $L^{p}$ is $p$-convex, by (1.7) we obtain that $\left(X^{q}\right)^{p}=\{0\}$. 
2.2.2. Type and cotype. To finish the chapter we will use the 2 -Köthe dual space in order to find a simple characterization for Hilbert spaces. The proof of the following theorem uses the type and cotype inequalities for $L^{p}$ spaces, which has type $\min \{2, p\}$ and cotype $\max \{2, p\}$ (see [24, Prop. 8.6] or [66, p. 73]). Recall that an AM-space $F$ is a Banach lattice such that satisfies $\|x \vee y\|_{F}=\max \left\{\|x\|_{F},\|y\|_{F}\right\}$ for $0<x, y \in F$. If the Banach lattice is a Banach function space $X$, then $(f \vee g)(\omega):=\max \{f(\omega), g(\omega)\}$ for $f, g \in X$. We show that we can provide a direct proof by using this abstract axiomatic definition, instead of writing the direct result for the case $X=L^{\infty}$, in such case $X^{p}=L^{p}$. This fact can be deduced also from Theorem 2.13, which implies that $X^{p}$ is $p$-concave when $X$ is an AM-space, thus $X^{p}$ is an $L^{p}$-space ([66, Cor. 1.d.12]), since $X^{p}$ is $p$-convex, but we do not use the Kakutani's representation. We will see in Corollary 2.18 that in fact, the AM-space involved is $L^{\infty}$.

Theorem 2.17. Let $1 \leq p \leq \infty$. Let $X$ be a Banach function space over a finite measure, which is an AM-space. Then $X^{p}$ has type $\min \{2, p\}$ and cotype $\max \{2, p\}$.

Proof. We consider separately type and cotype.

Type. Recall that the norm in $X^{p}$ is $\|f\|_{X^{p}}=\sup \left\{\|f g\|_{p}: g \in B_{X}\right\}$. If $f_{1}, \ldots, f_{n} \in X^{p}$ and $\varepsilon>0$ we claim that there exists $g \in B_{X}$ such that

$$
\left\|\sum_{k=1}^{n} r_{k}(t) f_{k}\right\|_{X^{p}} \leq\left\|g \sum_{k=1}^{n} r_{k}(t) f_{k}\right\|_{p}+\varepsilon^{2}, \quad t \in[0,1]
$$

where the election of $g \in B_{X}$ does not depends on $t \in[0,1]$. Let us define $\psi_{n}:[0,1] \rightarrow X^{p}$

$$
\psi_{n}(t):=\sum_{k=1}^{n} r_{k}(t) f_{k}
$$

By definition of the Rademacher functions, $\psi_{n}$ has at most $2^{n}$ values, since $r_{1}, \ldots, r_{n}$ are constant in each interval $\left[\frac{j}{2^{n}}, \frac{j+1}{2^{n}}\right)$ for $j=0, \ldots, 2^{n}-1$. Let us select $t_{j} \in\left[\frac{j}{2^{n}}, \frac{j+1}{2^{n}}\right)$ for $j=0, \ldots, 2^{n}-1$, (e.g. $t_{j}:=\frac{j}{2^{n}}$ ). Then $\psi_{n}(t)=\psi_{n}\left(t_{j}\right)$ for $t \in\left[\frac{j}{2^{n}}, \frac{j+1}{2^{n}}\right)$. By definition, for each $j \in\left\{0, \ldots, 2^{n}-1\right\}$ there exists $g_{j} \in B_{X}$ such that $\left\|\psi_{n}(t)\right\|_{X^{p}} \leq\left\|g_{j} \psi_{n}(t)\right\|_{p}+\varepsilon^{2}$ for every $t \in\left[\frac{j}{2^{n}}, \frac{j+1}{2^{n}}\right)$.

Let us define $g:=\left|g_{0}\right| \vee \cdots \vee\left|g_{2^{n}-1}\right|$. Since, $X$ is an AM-space we have that

$$
\|g\|_{X}=\max \left\{\left\|g_{1}\right\|_{X}, \ldots,\left\|g_{2^{n}-1}\right\|_{X}\right\} \leq 1 .
$$

On the other hand $\left|g_{j}\right| \leq|g|$, thus $\left|g_{j} \psi_{n}\left(t_{j}\right)\right| \leq\left|g \psi_{n}\left(t_{j}\right)\right|$ for all $j=0, \ldots, 2^{n}-1$. Then $\left|g_{j} \psi_{n}(t)\right| \leq\left|g \psi_{n}(t)\right|$ and $\left\|g_{j} \psi_{n}(t)\right\|_{p} \leq\left\|g \psi_{n}(t)\right\|_{p}$ for every $j=0, \ldots, 2^{n}-1, t \in\left[\frac{j}{2^{n}}, \frac{j+1}{2^{n}}\right)$. Let $t \in[0,1]$, then there exists $j$ such that $\psi_{n}(t)=\psi_{n}\left(t_{j}\right)$. Therefore (2.4) holds. 
If $1 \leq p \leq 2$ since $L^{p}$ has type $p$ we have

$$
\begin{aligned}
& \left(\int_{0}^{1}\left\|\sum_{k=1}^{n} r_{k}(t) f_{k}\right\|_{X^{p}}^{2} d t\right)^{1 / 2} \leq\left(\int_{0}^{1}\left\|\sum_{k=1}^{n} r_{k}(t) g f_{k}\right\|_{p}^{2} d t\right)^{1 / 2}+\left(\int_{0}^{1} \varepsilon^{2} d t\right)^{1 / 2} \\
& \leq C\left(\sum_{k=1}^{n}\left\|g f_{k}\right\|_{p}^{p}\right)^{1 / p}+\varepsilon \leq C\left(\sum_{k=1}^{n} \sup _{g \in B_{X}}\left\|g f_{k}\right\|_{p}^{p}\right)^{1 / p}+\varepsilon \leq C\left(\sum_{k=1}^{n}\left\|f_{k}\right\|_{X^{p}}^{p}\right)^{1 / p}+\varepsilon .
\end{aligned}
$$

Since $\varepsilon>0$ is arbitrary, we obtain that $X^{p}$ has type $p$. The case $p>2$ follows analogously applying that, in this case, $L^{p}$ has type 2 .

Cotype. Let $f_{1}, \ldots, f_{n} \in X^{p}$. For each $k \in\{1, \ldots, n\}$, choose $0 \leq g_{k} \in B_{X}$ such that $\left\|f_{k}\right\|_{X^{p}} \leq$ $\left\|g_{k} f_{k}\right\|_{p}+\frac{\varepsilon}{n^{1 / p}}$. Let us define $g_{0}:=g_{1} \vee \cdots \vee g_{n}$. Since $X$ is an AM-space, $g_{0} \in B_{X}, g_{i} \leq g_{0}$ and $\left|g_{i} f_{i}\right| \leq\left|g_{0} f_{i}\right|$, hence $\left\|g_{i} f_{i}\right\|_{p} \leq\left\|g_{0} f_{i}\right\|_{p}$ for $i=1, \ldots, n$. Therefore, for $p \geq 2$ applying that $L^{p}$ has cotype $p$ we have that

$$
\begin{aligned}
\left(\sum_{k=1}^{n}\left\|f_{k}\right\|_{X^{p}}^{p}\right)^{1 / p} & \leq\left(\sum_{k=1}^{n}\left(\left\|g_{0} f_{k}\right\|_{p}+\frac{\varepsilon}{n}\right)^{p}\right)^{1 / p} \leq\left(\sum_{k=1}^{n}\left\|g_{0} f_{k}\right\|_{p}^{p}\right)^{1 / p}+\varepsilon \\
& \leq C\left(\int_{0}^{1}\left\|g_{0} \sum_{k=1}^{n} r_{k}(t) f_{k}\right\|_{p}^{2} d t\right)^{1 / 2}+\varepsilon \\
& \leq C\left(\int_{0}^{1} \sup _{g \in B_{X}}\left\|g \sum_{k=1}^{n} r_{k}(t) f_{k}\right\|_{p}^{2} d t\right)^{1 / 2}+\varepsilon \\
& \leq C\left(\int_{0}^{1}\left\|\sum_{k=1}^{n} r_{k}(t) f_{k}\right\|_{X^{p}}^{2} d t\right)^{1 / 2}+\varepsilon,
\end{aligned}
$$

The case $p<2$ follows analogously applying that, in this case, $L^{p}$ has cotype 2 , which proves that $X^{p}$ has cotype $\min \{2, p\}$.

Corollary 2.18. Let $H$ be a Banach function space over a finite measure. Then $H$ is isomorphic to a Hilbert space if and only if there exists a Banach function AM-space X such that $H=X^{2}$.

Proof. Assume that $H$ is isomorphic to a Hilbert space, hence $H=H^{*}$. On one hand, let us prove that $H^{2}$ is an AM-space. By Proposition 2.4(3) taking $X:=H^{2}$ and $Y:=H$, we have trivially that $H \subseteq\left(H^{2}\right)^{2}$. Thus, by Lemma 2.2(2), Lemma 2.2(4) and Lemma 2.5(1) we have that

$$
H \subseteq\left(H^{2}\right)^{2} \subseteq L^{2},
$$

hence Lemma 2.5(2) (taking $X=H, q=1$ and $p=r=2$ ), implies that

$$
L^{2} \subseteq H^{\prime} \subseteq H^{*}=H .
$$

Then, $H=L^{2}$, thus $H^{2}=\left(L^{2}\right)^{2}=L^{\infty}$, which is an AM-space. On the other hand, inclusions (2.5) and (2.6) state that $H=\left(H^{2}\right)^{2}$. So choose $X:=H^{2}$.

For the converse, by the previous Theorem 2.17, we have that $X^{2}$ has type 2 and cotype 2. Applying Kwapień's Theorem we obtain the result. 
The Nikishin's Theorem provides the last application in the chapter. For this, let us present the Lorentz spaces. Let $(\Omega, \Sigma, \mu)$ be a $\sigma$-finite measure space and let $f \in L^{0}(\mu)$. See [8] for the following concepts: the distribution function $\mu_{f}:[0, \infty) \rightarrow[0, \infty]$ of a function $f \in L^{0}(\mu)$ is defined by $\mu_{f}(\lambda):=\mu(\{x \in \Omega:|f(x)|>\lambda\})$ and its decreasing rearrangement $f^{*}:[0, \infty) \rightarrow[0, \infty]$ is defined by

$$
f^{*}(t):=\inf \left\{\lambda \geq 0: \mu_{f}(\lambda) \leq t\right\} .
$$

Let $0<p, q \leq \infty$, the Lorentz space $L^{p, q}(\mu)$ is the class of functions in $L^{0}(\mu)$ such that the function norm $\|f\|_{p, q}<\infty$, where it is defined by

$$
\|f\|_{p, q}= \begin{cases}\left(\int_{0}^{\infty}\left(t^{1 / p} f^{*}(t)\right)^{q} \frac{d t}{t}\right)^{1 / q}, & \text { if } 0<p<\infty, 0<q<\infty, \\ \sup _{t>0} t^{1 / p} f^{*}(t), & \text { if } 0<p<\infty, q=\infty, \\ \|f\|_{\infty}, & \text { if } p=\infty, q=\infty .\end{cases}
$$

Corollary 2.19. Let $X$ and $Y$ be two Banach function spaces over a finite measure $\mu$, such that $X$ is order continuous. Let $T: X \rightarrow Y$ be a $\mu$-determined $p$-th power factorable operator. If $L^{1}\left(m_{T}\right)$ is $q$-concave for some $q<\infty$, then the (range) extension $X \stackrel{T}{\rightarrow} Y \stackrel{i}{\rightarrow} L^{0}$ factors through $L^{s, \infty}(\mu)$ where $s=\min \{2, p\}$.

PROOF. We can assume that $1 \leq p \leq 2$ since every $p$-th power factorable operator is $r$ th power factorable for every $1 \leq r \leq p$. It can be easily verified that $L^{p}\left(m_{T}\right)$ is $p q$-concave since $L^{1}\left(m_{T}\right)$ is $q$-concave (see e.g [79, Prop. 2.75(i)]). Applying [66, Prop. 1.f.3(ii)] we have that $L^{p}\left(m_{T}\right)$ has type $p$. Then we can assert that $L^{p}\left(m_{T}\right)$ has type $\min \{2, p\}$. Finally, we apply Nikishin's Theorem 1.10, in order to obtain that $i \circ I_{m_{T}}^{(p)}$ factors through the Lorentz space $L^{s, \infty}(\mu)$ where $s=\min \{2, p\}$, hence so does $i \circ T$. 

CHAPTER 3

\section{OPTIMAL RANGE THEOREM}

In Section 3.2 we will obtain a theorem that will allow us to define an embedding from a $q^{\prime}$-concave and Fatou Banach function space into $\left(L^{q}\left(m_{T^{\prime}}\right)\right)^{\prime}$ by an isometric map, where $m_{T^{\prime}}$ is the measure associated with the adjoint of a $q$-th power factorable operator $T$. In a sense, as we will see, the space $\left(L^{q}\left(m_{T^{\prime}}\right)\right)^{\prime}$ plays the role of optimal range, as the smaller $q^{\prime}$-concave Banach function space with the Fatou property, where the operator $T$ can take values. From this result we obtain several consequences, e.g. conditions for a map defined on $L^{\infty}$ to take necessarily values in $L^{\infty}$, and assuming some additional requirements, a characterization for $\left(L^{p}\left(m_{T^{\prime}}\right)\right)^{\prime}$ to be an almost $L^{p^{\prime}}$-space. Section 3.1 is devoted to analyze the duality relation between the spaces $L^{p}\left(m_{T}\right)$ and $L^{p}\left(m_{T^{\prime}}\right)$.

\subsection{The adjoint and Köthe adjoint operators}

Let us start by fixing the context that we need in order to define correctly the $p$-th power factorability of the Köthe adjoint operator of $T: E \rightarrow X$ where $E$ is a Banach space and $X$ is a quasi-Banach function space over a $\sigma$-finite measure space $(\Omega, \Sigma, \mu)$, with nontrivial and order continuous Köthe dual space.

(I) Let us compute the measure $m_{T^{\prime}}$ associated with $T^{\prime}$.

$$
\begin{aligned}
& T^{\prime}: X^{\prime} \longrightarrow E^{*} \\
& g \longmapsto\left\{\begin{array}{rll}
T^{\prime}(g): \quad E & \rightarrow \mathbb{R} \\
x & \mapsto\langle T(x), g\rangle=\int_{\Omega} g T(x) d \mu=\left(I_{\mu} \circ M_{g} \circ T\right)(x) .
\end{array}\right.
\end{aligned}
$$

We thus get $m_{T^{\prime}}: \Sigma \rightarrow E^{*}$ defined by $m_{T^{\prime}}(A)=T^{\prime}\left(\chi_{A}\right)$, i.e.

$$
\left(m_{T^{\prime}}(A)\right)(x)=\left(T^{\prime}\left(\chi_{A}\right)\right)(x)=\int_{A} T(x) d \mu, \quad x \in E .
$$

or in other words $m_{T^{\prime}}(A)=I_{\mu} \circ M_{\chi_{A}} \circ T$.

(II) About the $\mu$-determination of $T^{\prime}$. If $\chi_{\Omega} \in T(E)$, then there exists $x_{0} \in E$ such that $T\left(x_{0}\right)=\chi_{\Omega} \in X$. So we claim that $\mathscr{N}_{0}\left(m_{T^{\prime}}\right) \subseteq \mathscr{N}_{0}(\mu)$. Assume that $A \in \Sigma$ is $m_{T^{\prime}}$-null, this implies that $m_{T^{\prime}}(B)=T^{\prime}\left(\chi_{B}\right)=0$ for all $B \in 2^{A} \cap \Sigma$. Hence, by (3.1), $\int_{B} T(x) d \mu=0$ for every $x \in E$ and every $B \in 2^{A} \cap \Sigma$. So for every $B \in 2^{A} \cap \Sigma$ we have

$$
0=\int_{B} T\left(x_{0}\right) d \mu=\int_{B} \chi_{\Omega} d \mu=\int_{\Omega} \chi_{B} d \mu=\mu(B) .
$$


Therefore $\mathscr{N}_{0}\left(m_{T^{\prime}}\right) \subseteq \mathscr{N}_{0}(\mu)$.

Recall that the requirement on the $\mu$-determination for the operator $T$ is imposed in order to obtain that its domain space will be injectively included in its optimal domain $L^{1}\left(m_{T}\right)$ (see [79, Prop. 4.4(iii)]). However, it is not necessary for obtaining a factorization of $T$ through this space.

The following lemma will allow us to obtain a representation of the adjoint operator of the integration map associated with a vector measure, as a Radon-Nikodým derivative.

Lemma 3.1. Let $X$ and $Y$ be two order continuous Banach function spaces over $\mu$, such that $X^{\prime}$ and $Y^{\prime}$ are also order continuous. Let $T: X \rightarrow Y$ be an operator such that there exist a Banach space $E$ and operators $R: X \rightarrow E$ and $S: E \rightarrow Y$ such that $T=S \circ R$. Then

$$
\int_{\Omega} g d m_{T^{\prime}}=\frac{d\left\langle m_{R}, \int_{\Omega} g d m_{S^{\prime}}\right\rangle}{d \mu}, \quad g \in L^{1}\left(m_{S^{\prime}}\right) .
$$

Proof. Thanks to the order continuity assumptions, the given factorization for $T$ and $T^{\prime}$ are
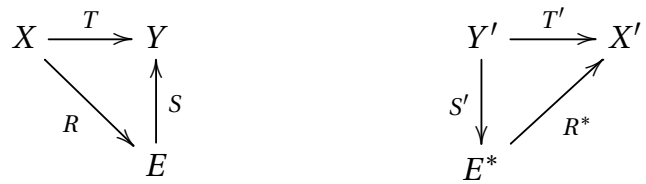

Let $x^{*} \in E^{*}$. Then the measure defined as $\left\langle m_{R}, x^{*}\right\rangle$ is absolutely continuous with respect to $\mu$ (Proposition 1.1(2)). Observe that $m_{T^{\prime}}(A)=m_{R^{*} \circ S^{\prime}}(A)=R^{*}\left(m_{S^{\prime}}(A)\right)$, by the continuity of $R^{*}$ and the order continuity of the Banach function space $L^{1}\left(m_{S^{\prime}}\right)$, we obtain that

$$
\int_{\Omega} g d m_{\left(R^{*} \circ S^{\prime}\right)}=R^{*}\left(\int_{\Omega} g d m_{S^{\prime}}\right), \quad g \in L^{1}\left(m_{S^{\prime}}\right) .
$$

Let be $f \in X$ and $g \in L^{1}\left(m_{S^{\prime}}\right)$. Then we have that $\int_{\Omega} g d m_{S^{\prime}} \in E^{*}$, and hence

$$
\begin{aligned}
\left\langle f, \int_{\Omega} g d m_{T^{\prime}}\right\rangle & =\left\langle f, \int_{\Omega} g d m_{\left(R^{*} \circ S^{\prime}\right)}\right\rangle=\left\langle f, R^{*}\left(\int_{\Omega} g d m_{S^{\prime}}\right)\right\rangle \\
& =\left\langle R f, \int_{\Omega} g d m_{S^{\prime}}\right\rangle=\left\langle\int_{\Omega} f d m_{R}, \int_{\Omega} g d m_{S^{\prime}}\right\rangle \\
& =\int_{\Omega} f \frac{d\left\langle m_{R}, \int_{\Omega} g d m_{S^{\prime}}\right\rangle}{d \mu} d \mu=\left\langle f, \frac{d\left\langle m_{R}, \int_{\Omega} g d m_{S^{\prime}}\right\rangle}{d \mu}\right\rangle .
\end{aligned}
$$

Consequently

$$
\int_{\Omega} g d m_{T^{\prime}}=\frac{d\left\langle m_{R}, \int_{\Omega} g d m_{S^{\prime}}\right\rangle}{d \mu}, \quad g \in L^{1}\left(m_{S^{\prime}}\right) .
$$

Remark 3.2. In particular, under the assumptions of the lemma above for $X$ and $Y$, we have the trivial factorization $T=\operatorname{Id}_{Y} \circ T$. If $T^{\prime}$ is $\mu$-determined, then

$$
\int_{\Omega} g d m_{T^{\prime}}=\frac{d\left\langle m_{T}, g\right\rangle}{d \mu}, \quad g \in Y^{\prime},
$$


since $\left(\operatorname{Id}_{Y}\right)^{\prime}=\operatorname{Id}_{Y^{\prime}}$, and so $\int_{\Omega} g d m_{\operatorname{Id}_{Y^{\prime}}}=\operatorname{Id}_{Y^{\prime}}(g)=g$ for every $g \in Y^{\prime}$. If in addition $X$ and $Y$ are Fatou and $T$ is $\mu$-determined, then $T^{\prime \prime}=T$, which implies that

$$
\int_{\Omega} f d m_{T}=\frac{d\left\langle m_{T^{\prime}}, f\right\rangle}{d \mu}, \quad f \in X=X^{\prime \prime} .
$$

Let us now consider a Banach space $E$ and an operator $T: X \rightarrow E$ defined on an order continuous Banach function space $X$ over $\mu$. Let us define the map $D_{m_{T}}: E^{*} \rightarrow\left(L^{1}\left(m_{T}\right)\right)^{\prime}$ as

$$
D_{m_{T}}\left(x^{*}\right):=\frac{d\left\langle m_{T}, x^{*}\right\rangle}{d \mu}
$$

which is well defined since $\left|\left\langle m_{T}, x^{*}\right\rangle\right| \ll\left\|m_{T}\right\| \ll \mu$ and the Radon-Nikodým derivative exists. Note that $D_{m_{T}}$ is linear and continuous. Let us show that $D_{m_{T}}$ is in fact the adjoint operator of $I_{m_{T}}$. Let $f \in L^{1}\left(m_{T}\right)$ and $x^{*} \in E^{*}$, then

$$
\left\langle I_{m_{T}} f, x^{*}\right\rangle=\int_{\Omega} f d\left\langle m_{T}, x^{*}\right\rangle=\int_{\Omega} f \frac{d\left\langle m_{T}, x^{*}\right\rangle}{d \mu} d \mu=\int_{\Omega} f D_{m_{T}}\left(x^{*}\right) d \mu=\left\langle f, D_{m_{T}}\left(x^{*}\right)\right\rangle,
$$

and thus $D_{m_{T}}=\left(I_{m_{T}}\right)^{\prime}$.

Remark 3.3. Let $X$ be an order continuous Banach function space over a finite measure $\mu$ and let $E$ be a Banach space. Let $T: X \rightarrow E$ be an operator. It is called inclusion/quotient map, the operator $[i]: X \rightarrow L^{1}\left(m_{T}\right)$ defined by $[i](f):=[f]_{m_{T}}$, where $f \in X$. The map [i] depends on $\mu$ and $m_{T}$, but these measures are not in general equivalent. Observe that for different Rybakov measures $v$ of $m_{T}$, we can obtain different representations of $[i]^{\prime}:\left(L^{1}\left(m_{T}\right)\right)^{\prime} \rightarrow X^{\prime}$. For such $v$, for $f \in X$ and $g \in\left(L^{1}\left(m_{T}\right)\right)^{\prime}$ we have

$$
\left\langle f,[i]^{\prime}(g)\right\rangle=\langle[i](f), g\rangle=\int_{\Omega}[i](f) g d v=\int_{\Omega}[i](f) g \frac{d v}{d \mu} d \mu=\left\langle[i](f), g \frac{d v}{d \mu}\right\rangle .
$$

Let us denote this adjoint map $[i]^{\prime}$ by “ $\left.\hookrightarrow[i]\right]^{\prime}$. The following diagrams always hold
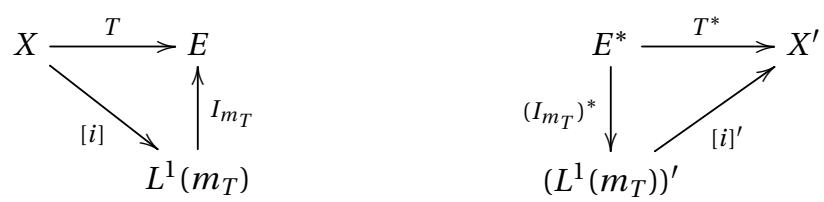

DIAGRAM 3.1. Factorization through the inclusion/quotient map

\subsection{Optimal range theorem}

In this section we obtain a representation of the optimal Fatou Banach function space in which the range of a given operator is included. Let us start by some examples of operators that satisfy that their adjoint maps are $p$-th power factorable for some $p>1$. 
Example 3.4 (Hardy type operator). Let $s>0$ and consider the kernel operator $H_{s}$ with kernel function $K(x, y):=\frac{1}{x^{s}} \chi_{[0, x]}(y)$. If $1 \leq u \leq v$ and $1 \leq v<\frac{1}{s}$, the kernel operator $H_{s}: L^{u}[0,1] \rightarrow L^{v}[0,1]$ is well defined (and continuous). We have that

$$
H_{s}(f)(x)=\int_{0}^{1} K(x, y) f(y) d y=\int_{0}^{1} \frac{1}{x^{s}} f(y) \chi_{[0, x]}(y) d y=\frac{1}{x^{s}} \int_{0}^{x} f(y) d y .
$$

Since for $x, y \in[0,1], \chi_{[0, x]}(y)=\chi_{[y, 1]}(x)$ the adjoint map $\left(H_{s}\right)^{\prime}: L^{v^{\prime}}[0,1] \rightarrow L^{u^{\prime}}[0,1]$ is given by

$$
\left(H_{s}\right)^{\prime}(g)(y)=\int_{0}^{1} \frac{1}{x^{s}} \chi_{[0, x]}(y) g(x) d x .
$$

If $g \in L^{v^{\prime}}[0,1]$, using Minkowski's integral inequality (1.8) and Hölder-Rogers inequality, we obtain

$$
\begin{gathered}
\left\|\left(H_{S}\right)^{\prime}(g)\right\|_{L^{u^{\prime}}}=\left(\int_{0}^{1}\left|\int_{0}^{1} \chi_{[0, x]}(y) \frac{g(x)}{x^{s}} d x\right|^{u^{\prime}} d y\right)^{1 / u^{\prime}} \leq \int_{0}^{1}\left(\int_{0}^{1} \chi_{[0, x]}(y)\left|\frac{g(x)}{x^{s}}\right|^{u^{\prime}} d y\right)^{1 / u^{\prime}} d x \\
=\int_{0}^{1}\left|g(x)\left\|x^{1-s} \mid d x \leq\right\| x^{1-s}\left\|_{L^{\left(v^{\prime} / q\right)^{\prime}}}\right\| g\left\|_{L^{v^{\prime} / q}}=\left(\frac{v^{\prime}-q}{v^{\prime}(2-s)-q}\right)^{\frac{v^{\prime}-q}{v^{\prime}}}\right\| g \|_{\left(L^{v^{\prime}}\right)_{[q]} .}\right.
\end{gathered}
$$

Thus, $\left(H_{s}\right)^{\prime}$ is $q$-th power factorable for $1 \leq q<v^{\prime}(2-s)$.

The case $H_{0}$ gives the Volterra operator. It is well known when this operator is $p$-th power factorable for $1 \leq p<u$ (see [79, Ex. 5.9]). We have shown in this example when this condition holds for the adjoint map $\left(H_{0}\right)^{\prime}$. See Example 3.14 for information about its optimal range.

Example 3.5 (Convolution operator). Let $G$ be a compact Hausdorff abelian group with normalized Haar measure $\mu$ defined on the Borelian sets of $G(\mathscr{B}(G))$. Let $\lambda$ be a regular measure on $\mathscr{B}(G)$. We say that $\lambda$ is a $L^{q}$-improving measure $(q \geq 1)$ if there exists $r \in(q, \infty)$ such that $f * \lambda \in L^{r}(G)$ for all $f \in L^{q}(G)$. It is well known that there is a direct relation between $L^{q}$-improving measures and $p$-th power factorable convolution operators (see [79, Sect.7.5]). If $h \in L^{1}(G)$ we can always consider the measure $\mu_{h}(A):=\int_{A} h d \mu$. For this kind of measures, the fact that $h$ belongs to a particular $L^{s}(G)$-space determines if it is $L^{q}$ improving, and also that the corresponding convolution operator is $p$-th power factorable for a certain $p$.

Let $1<p<\infty$ and consider the convolution operator $C_{h}^{(p)}: L^{p}(G) \rightarrow L^{p}(G)$ given by $C_{h}^{(p)}(f):=f * \mu_{h}$, that is continuous, and the reflection measure of $\lambda$ defined by $R \lambda(A):=$ $\lambda(-A)$. Note that for measures $\lambda(A):=\int_{A} h(x) d \mu$ we always have $R \lambda(A)=\int_{A} h(-x) d \mu$. Using Fubini's Theorem, we obtain that the adjoint operator $\left(C_{h}^{(p)}\right)^{\prime}: L^{p^{\prime}}(G) \rightarrow L^{p^{\prime}}(G)$ is given by $\left(C_{h}^{(p)}\right)^{\prime}(g)=g * R \mu_{h}$. Thus, we can apply [79, Prop. 7.96] taking into account that all $L^{s}(G)$ are rearrangement invariant: for $h \in L^{r}(G) \backslash L^{p^{\prime}}(G)\left(1<r<p^{\prime}\right)$ and $u \in\left(1, p^{\prime}\right)$ such that $\frac{1}{u}+\frac{1}{r}=\frac{1}{p^{\prime}}+1,\left(C_{h}^{(p)}\right)^{\prime}$ is $\left(p^{\prime} / u\right)$-th power factorable.

The following provides an example of $p$-th power factorable kernel operator with $q$-th power factorable adjoint operator. 
Example 3.6 (Degenerate or separable kernel). Let $(\Omega, \Sigma, \mu)$ be a finite measure space. Let $X$ and $Y$ be Banach function spaces defined over $\mu$. The separable kernel is defined by

$$
K(x, y):=\sum_{i=1}^{n} a_{i}(x) b_{i}(y), \quad \text { for } x, y \in \Omega,
$$

where $a_{i} \in Y$ and $b_{i} \in X^{\prime}$ for $i=1, \ldots, n$, then the associated kernel operator $T_{K}$ maps $X$ into $Y$ (see [108, p. 210 and Ch. 13, §95] where this kernel operator is called a kernel operator of finite rank). These kernels are useful, for instance in the Tikhonov approximation of the solutions of integral equations (see e.g. [57]).

Let $1<p, q, r, s<\infty$ be such that $1<p<r$ and $1<q<s^{\prime}$. Let $0<k_{1} \in L^{u}[0,1]$ and $0<k_{2} \in L^{v}[0,1]$, where $u:=\left(s^{\prime} / q\right)^{\prime}$ and $v:=(r / p)^{\prime}$. Let us define the kernel function

$$
K(x, y):=k_{1}(x) k_{2}(y) \in L^{1}([0,1] \times[0,1]) .
$$

On one hand, thanks to the comments above, the kernel operator associated with $K$ is $T_{K}: L^{v^{\prime}}[0,1] \rightarrow L^{u}[0,1]$. On the other hand, since $r / p<r$, we have that $v^{\prime}<r$, thus $L^{r}[0,1] \subseteq L^{v^{\prime}}[0,1]$. Also, since $q<s^{\prime}$ we have $s<q^{\prime}$, and so $s<\left(s^{\prime} / q\right)^{\prime}=u$, which implies that $L^{u}[0,1] \subseteq L^{s}[0,1]$.

Now, let us consider $T_{K}: L^{r}[0,1] \rightarrow L^{s}[0,1]$ be the operator associated with $K$. Let us see that $T_{K}$ is $p$-th power factorable and $T_{K}^{\prime}$ is $q$-th power factorable.

Let $f \in L^{r}[0,1]$ and $g \in L^{s^{\prime}}[0,1]$. By the Hölder-Rogers inequality we have

$$
\begin{aligned}
\left\|T_{K} f\right\|_{s} & =\left(\int_{0}^{1}\left|\int_{0}^{1} k_{1}(x) k_{2}(y) f(y) d y\right|^{s} d x\right)^{1 / s} \\
& \leq\left\|k_{1}\right\|_{s}\left\|k_{2} f\right\|_{1} \leq\left\|k_{1}\right\|_{u}\left\|k_{2}\right\|_{(r / p)^{\prime}}\|f\|_{r / p} \leq D\|f\|_{r / p}<\infty, \\
\left\|T_{K}^{\prime} g\right\|_{r^{\prime}} & =\left(\int_{0}^{1}\left|\int_{0}^{1} k_{2}(y) k_{1}(x) g(x) d x\right|^{r^{\prime}} d y\right)^{1 / r^{\prime}} \\
& \leq\left\|k_{2}\right\|_{r^{\prime}}\left\|k_{1} g\right\|_{1} \leq\left\|k_{2}\right\|_{v}\left\|k_{1}\right\|_{\left(s^{\prime} / q\right)^{\prime}}\|g\|_{s^{\prime} / q} \leq D\|g\|_{s^{\prime} / q}<\infty,
\end{aligned}
$$

where $D:=\left\|k_{1}\right\|_{u}\left\|k_{2}\right\|_{v}$ and so we establish the statement.

The idea of the main theorem in this chapter is to dualize the diagram of a $p$-th power factorable adjoint operator. This scheme will allow us to compute in some cases the smaller Banach function space where a given operator takes its values.

Theorem 3.7. Let $1 \leq p<\infty$. Let $X$ be a Fatou Banach function space over a finite measure $\mu$ such that $X^{\prime}$ is order continuous. Let $T: E \rightarrow X$ be an operator from a Banach space $E$ to $X$ with $p$-th power factorable adjoint. Then $T$ factors through $\left(L^{p}\left(m_{T^{\prime}}\right)\right)^{\prime}$. Furthermore, if there is a Banach function space $Z$ such that

(i) $T(E) \subseteq Z \subseteq X$,

(ii) $Z$ is Fatou, $Z$ ' is order continuous and

(iii) the (range) restriction $S: E \rightarrow Z$ of $T$ has $p$-th power factorable adjoint, 
then $\left(L^{p}\left(m_{T^{\prime}}\right)\right)^{\prime} \hookrightarrow_{[i]^{\prime}} Z$.

Proof. Since $T^{\prime}$ is $p$-th power factorable, there is a constant $K>0$ such that for every $x \in E$,

$$
|\langle T(x), g\rangle| \leq K\|x\|_{E}\|g\|_{\left(X^{\prime}\right)_{[p]}}
$$

for all $x \in E$ and $g \in X^{\prime}$. Since $X^{\prime}$ is order continuous, this implies that the Köthe adjoint map $T^{\prime}$ factors as follows

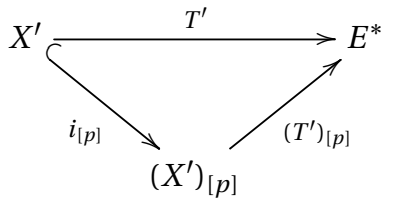

where $i_{[p]}$ is the natural continuous inclusion and $\left(T^{\prime}\right)_{[p]}$ is the extension of $T^{\prime}$. The order continuity of $X^{\prime}$ gives also that the expression $m_{T^{\prime}}(A)=T^{\prime}\left(\chi_{A}\right), A \in \Sigma$, defines a vector measure. Applying Proposition 1.8 we can assert that

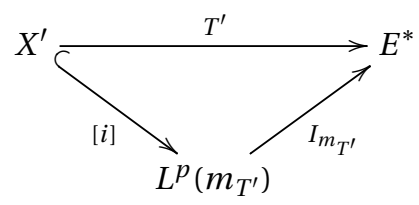

DIAGRAM 3.2. Optimal domain for the adjoint operator

where [i] is the inclusion/quotient map and $I_{m_{T^{\prime}}}$ is the integration map (see [79, Sect. 5.2] and also [13, Prop. 4] for the case when $[i]$ is not injective). Dualizing this last factorization diagram, taking into account that $X^{\prime *}=X^{\prime \prime}=X$, we obtain the following diagram,

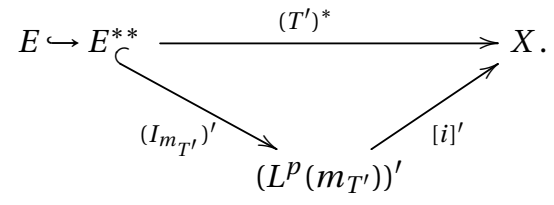

These arguments give the factorization through $\left(L^{p}\left(m_{T^{\prime}}\right)\right)^{\prime}$. For the second assertion, by hypothesis, the range of $T$ lies in $Z \subseteq X$. Then $T^{\prime}$ factors through $Z^{\prime}$ and by hypothesis $S^{\prime}$ is $p$-th power factorable. This implies that $S^{\prime}$ factors through $L^{p}\left(m_{S^{\prime}}\right)$. But note that $m_{S^{\prime}}=$ $m_{T^{\prime}}$. Consequently, by Proposition 1.8, $[i]\left(Z^{\prime}\right) \subseteq L^{p}\left(m_{T^{\prime}}\right)$, this bring us to the conclusion that $\left(L^{p}\left(m_{T^{\prime}}\right)\right)^{\prime} \hookrightarrow[i]^{\prime} Z^{\prime \prime}=Z$.

The following result provides some structure information for the space $\left(L^{p}\left(m_{T^{\prime}}\right)\right)^{\prime}$ without any assumption on the $p$-th power factorability of $T^{\prime}$. 
Corollary 3.8. Assume that $X$ is a Fatou and order continuous Banach function space over a finite measure, such that $X^{\prime}$ is order continuous. Consider $T: E \rightarrow X$ an operator. Then $\left(L^{p}\left(m_{T^{\prime}}\right)\right)^{\prime} \hookrightarrow_{[i]^{\prime}}\left(X^{p}\right)^{\prime}$. Moreover, the optimal range in the sense of Theorem 3.7 of the (range) extension $\widehat{T}: E \rightarrow X \hookrightarrow\left(X^{p}\right)^{\prime}$ of $T$ is the space $\left(L^{p}\left(m_{T^{\prime}}\right)\right)^{\prime}$.

Proof. Notice that the requirement of that $X^{p}$ to be a Banach function space is fulfilled, since $X$ has weak order unit (see [71, Prop. 2] and [13, p. 90]). By Proposition 2.8, $T^{p}: X^{p} \rightarrow E^{*}$ is $p$-th power factorable, then $X^{p} \hookrightarrow_{[i]} L^{p}\left(m_{T^{p}}\right)=L^{p}\left(m_{T^{\prime}}\right)$ since $m_{T^{p}}=m_{T^{\prime}}$ (Proposition 1.8). Dualizing this inclusion/quotient map we obtain the first assertion. By [12, Prop. 3.3(a) and (b)] we have that $X^{p}$ is Fatou. Then $X^{p}=\left(X^{p}\right)^{\prime \prime}$, in this way we have that $T^{p}=\widehat{T}^{\prime}:\left(X^{p}\right)^{\prime \prime}=X^{p} \hookrightarrow X^{\prime} \stackrel{T^{\prime}}{\rightarrow} E^{*}$, thus $m_{\widehat{T}^{\prime}}=m_{T^{\prime}}$. Therefore $\left(L^{p}\left(m_{\widehat{T}^{\prime}}\right)\right)^{\prime}=\left(L^{p}\left(m_{T^{\prime}}\right)\right)^{\prime}$, which is the optimal range of $T^{\prime}$ (in the sense given in Theorem 3.7).

Remark 3.9. If we rewrite Theorem 3.7 for $p=1$, we do not assume any condition for the adjoint map. In such case, we have an optimal range for continuous operators. It can be formulated as follows. Assume $X$ is a Banach function space with the Fatou property such that $X^{\prime}$ is order continuous. Let $T: E \rightarrow X$. Therefore $T$ factors through $\left(L^{1}\left(m_{T^{\prime}}\right)\right)^{\prime}$, if the range of $T$ lies into a Banach function space $Z \subseteq X$ such that $Z$ has the Fatou property and $Z^{\prime}$ is order continuous, then $\left(L^{1}\left(m_{T^{\prime}}\right)\right)^{\prime} \hookrightarrow_{[i]^{\prime}} Z$. If $T$ is a $\mu$-determined operator we obtain a natural inclusion, as we seen in Remark 3.3 there is $h=\frac{d v}{d \mu}$ such that $M_{h}\left(L^{1}\left(m_{T^{\prime}}\right)^{\prime}\right) \subseteq Z$.

3.2.1. Applications of the optimal range theorem. In order to find applications let us "approximate", in some sense, the optimal range for operators from an AM-space into a Banach function space. We also compute the optimal range for operators that has compact associated integration map [79, Sect. 3.3], always assuming that the adjoint operator is $p$-th power factorable.

Let $(\Omega, \Sigma, \mu)$ be a measure space. Let $A \in \Sigma$, let us define the restriction $\left(\Omega \cap A,\left.\Sigma\right|_{A},\left.\mu\right|_{A}\right)$ to $A$ of this measure space, where $\left.\Sigma\right|_{A}:=\{B \cap A: B \in \Sigma\}$ which is a $\sigma$-algebra on $A$ and also let the measure $\left.\mu\right|_{A}(B):=\mu(B \cap A)$, where $\left.B \in \Sigma\right|_{A}$. Hence, given a Banach function space $X$ over $\mu$, we define $\left.X\right|_{A}$ the space of restrictions to $A$ of functions in $X$. We will say that a Banach function space $X$ is almost an $L^{p}$-space if for every $\varepsilon>0$, there exists a measurable set $A_{\varepsilon} \in \Sigma$ such that $\mu\left(A_{\varepsilon}\right)<\varepsilon$ and such that the restriction $\left.X\right|_{\Omega \backslash A_{\varepsilon}}$ [79, Prop. 4.28] is order isomorphic to an $L^{p}$-space.

Theorem 3.10. Let $p>1$. Consider $(\Omega, \Sigma, \mu)$ a finite measure space, a Banach function space $F(\mu)$ and an operator $T: L^{\infty}(v) \rightarrow F$, where $v$ is a $\sigma$-finite measure. Assume that $F$ has the Fatou property and $F^{\prime}$ is order continuous, $T^{\prime}$ is a positive $p$-th power factorable operator such that $T^{\prime}\left(F^{\prime}\right) \subseteq L^{1}(v)$. Then the (optimal) range $\left(L^{p}\left(m_{T^{\prime}}\right)\right)^{\prime}$ of $T$ is almost an $L^{p^{\prime}}$-space. 
Proof. Since $F^{\prime}$ is order continuous, so is $T^{\prime}: F^{\prime} \rightarrow L^{1}(v)$ and $m_{T^{\prime}}$ is a countably additive vector measure. Then, by Proposition 1.8, $T^{\prime}$ can be factored through the space $L^{p}\left(m_{T^{\prime}}\right)$ by means of a inclusion/quotient map [i] (as we showed when we started the chapter, see also [13, Prop. 4] and [79, Ch. 5]) as follows

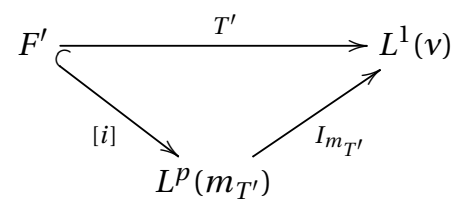

Step 1. The integration operator $I_{m_{T^{\prime}}}$ is a positive map (since $T^{\prime}$ is positive and $[i]\left(F^{\prime}\right)$ is dense in the $p$-convex space $\left.L^{p}\left(m_{T^{\prime}}\right)\right)$ ). Moreover $L^{1}(v)$ is $p$-concave for every $p \geq 1$, hence $I_{m_{T^{\prime}}}$ is $p$-concave (see [66, Th. 1.d.9] and comments below it). By applying the variant of the Maurey-Rosenthal's Theorem given in [79, Th. 6.41(iii)], this implies that there exists $\mu_{0}$, a Rybakov measure for $m_{T^{\prime}}$, such that $T^{\prime}$ can be extended to $L^{p}\left(\mu_{0}\right)$ as $T^{\prime}=S_{0} \circ i$ where $S_{0}$ is the extension of the integration map. This gives an extension of $I_{m_{T^{\prime}}}$ to $L^{p}\left(\mu_{0}\right)$ as

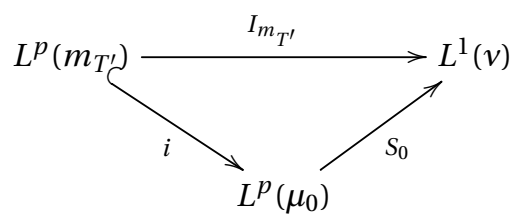

Step 2. Let us now show that a restriction of $S_{0}$ to the complementary of a set as small in measure as we want is $p$-th power factorable. For doing this, just take into account that the vector measure $m_{S_{0}}$ coincides with $m_{T^{\prime}}$. In particular, it is positive and 1-concave. Again the variant of the Maurey-Rosenthal's Theorem quoted above gives (for $p=1$ ) that $S_{0}: L^{p}\left(\mu_{0}\right) \rightarrow L^{1}(v)$ can be extended to the space $L^{1}(\eta)$, where $\eta$ is a Rybakov measure for $m_{S_{0}}$ and so for $m_{T^{\prime}}$. More precisely, by definition of the norm (1.5), it can be factored through the inclusion map $L^{1}\left(m_{S_{0}}\right) \hookrightarrow L^{1}(\eta)$. In fact we extend the integration map of the measure $m_{S_{0}}$.

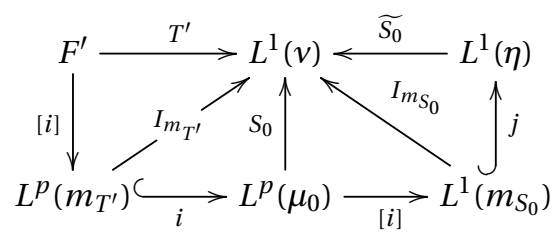

DIAGRAM 3.3. Characterization for the optimal range

In consequence there is the Radon-Nikodým derivative $h=\frac{d \eta}{d \mu_{0}}$ such that for every $f \in L^{p}\left(\mu_{0}\right)$

$$
\begin{aligned}
\left\|S_{0} f\right\|_{L^{1}(v)} & =\left\|\widetilde{S_{0}} \circ j \circ[i] f\right\|_{L^{1}(v)} \leq\left\|\widetilde{S_{0}}\right\|\|j([i] f)\|_{L^{1}(\eta)} \\
& \leq\left\|\widetilde{S_{0}}\right\| \int_{\Omega}|f| d \eta=\left\|\widetilde{S_{0}}\right\|\left\||h|^{1 / p}|f|^{1 / p}\right\|_{L^{p}\left(\mu_{0}\right)}^{p}
\end{aligned}
$$


The function $h$ is integrable with respect to $\mu_{0}$, then $|h|^{1 / p} \in L^{p}\left(\mu_{0}\right) \subseteq L^{1}\left(\mu_{0}\right)$. Since this $\mu_{0}$ is a Rybakov measure for $m_{T^{\prime}}$, by definition of semivariation (1.1), it is equivalent to the semivariation $\left\|m_{T^{\prime}}\right\|$. Fix $\varepsilon>0$. Thus, since $|h|^{1 / p}$ is integrable with respect to $\mu_{0}$, in particular it is $\mu_{0}$-measurable, then we have that there is a constant $K_{\varepsilon}$ such that $\left\|m_{T^{\prime}}\right\|\left(A_{\varepsilon}\right)<\varepsilon$, where $A_{\varepsilon}:=\left\{\omega \in \Omega:|h(\omega)|^{1 / p}>K_{\varepsilon}\right\}$. Then

$$
\left\||h|^{1 / p}|f|^{1 / p}\right\|_{L^{p}\left(\mu_{0}||_{\varepsilon}^{c}\right)}^{p} \leq K_{\varepsilon}\left\||f|^{1 / p}\right\|_{L^{p}\left(\mu_{0}||_{A_{\varepsilon}^{c}}\right)}^{p},
$$

where $A_{\varepsilon}^{c}:=\Omega \backslash A_{\varepsilon}$, i.e. the restriction of $S_{0}$ to this set is $p$-th power factorable, i.e.

$$
S_{0}: L^{p}\left(\left.\mu_{0}\right|_{A_{\varepsilon}^{c}}\right) \hookrightarrow{ }_{[i]} L^{p}\left(\left.m_{S_{0}}\right|_{A_{\varepsilon}^{c}}\right) \rightarrow L^{1}(v),
$$

(notice that $\left\|m_{T^{\prime}}\right\|$ is equivalent to $\mu$, so the condition $\left\|m_{T^{\prime}}\right\|\left(A_{\varepsilon}\right)<\varepsilon$ can be written in terms of $\mu$ ). The arguments in Theorem 3.7 on the optimal domain for $T^{\prime}$ can then be applied. As we said, $m_{T^{\prime}}=m_{S_{0}}$ and $S_{0}$ is $\mu_{0}$-determined, then

$$
L^{p}\left(\left.m_{T^{\prime}}\right|_{A_{\varepsilon}^{c}}\right) \subseteq L^{p}\left(\left.\mu_{0}\right|_{A_{\varepsilon}^{c}} \subseteq L^{p}\left(\left.m_{S_{0}}\right|_{A_{\varepsilon}^{c}}\right)=L^{p}\left(\left.m_{T^{\prime}}\right|_{A_{\varepsilon}^{c}}\right) .\right.
$$

So for every $\varepsilon>0$ there exists $A_{\varepsilon} \in \Sigma$ such that $\left(L^{p}\left(\left.m_{T^{\prime}}\right|_{A_{\varepsilon}^{c}}\right)\right)^{\prime}=L^{p^{\prime}}\left(\left.\mu_{0}\right|_{A_{\varepsilon}^{c}}\right)$.

The next theorem shows, for $p=1$, that the optimal range is exactly an $L^{\infty}$-space.

Theorem 3.11. Consider a finite measure space $(\Omega, \Sigma, \mu)$, a Banach function space $F(\mu)$ and an operator $T: L^{\infty}(v) \rightarrow F$, where $v$ is a $\sigma$-finite measure. Suppose that $F$ has the Fatou property, $F^{\prime}$ is order continuous, $T^{\prime}$ is positive, $\mu$-determined and $T^{\prime}\left(F^{\prime}\right) \subseteq L^{1}$. Then the optimal range of $T$ is $L^{\infty}(\mu)$.

PRoof. The proof is the same as in the previous theorem, but the second step in the proof is not needed. In this case we obtain

$$
L^{1}\left(m_{T^{\prime}}\right) \subseteq L^{1}\left(\mu_{0}\right) \subseteq L^{1}\left(m_{S_{0}}\right)=L^{1}\left(m_{T^{\prime}}\right) .
$$

Taking into account that $\mu$ and $\mu_{0}$ are equivalent, the optimal range $\left(L^{1}\left(m_{T^{\prime}}\right)\right)^{\prime}$ given by Theorem 3.7 coincides with $L^{\infty}(\mu)$.

Proposition 3.12. Let $1 \leq p<\infty$. Let us consider an operator $T: X(\mu) \rightarrow Y(v)$ between Banach function spaces, where $Y^{\prime}(v)$ is order continuous. Suppose that

$$
H:=\left\{T^{\prime}(g): g \in B_{Y^{\prime}} \text { and } \sup _{A \in \Sigma}\left\|T^{\prime}\left(g \chi_{A}\right)\right\|_{X^{*}} \leq 1\right\} \subseteq X^{*}
$$

is a relatively compact and that $T^{\prime}$ is $p$-th power factorable. Then the optimal range in the sense of Theorem 3.7, $L^{p}\left(m_{T^{\prime}}\right)^{\prime}$ is order isomorphic to an $L^{p^{\prime}}$-space.

Proof. Since $Y^{\prime}$ is order continuous, the operator $T^{\prime}: Y^{\prime} \rightarrow X^{*}$ defines a countably additive vector measure and simple functions are dense in both $Y^{\prime}$ and $L^{1}\left(m_{T^{\prime}}\right)$. This, together with the condition on $H$ implies that the integration map $I_{m_{T^{\prime}}}: L^{1}\left(m_{T^{\prime}}\right) \rightarrow X^{*}$ is compact (recall (1.6) the equivalent norm $\|\cdot\|_{L^{p}(m)}$ for the spaces $L^{p}(m)$ ). In this case, it is well known that the space $L^{1}\left(m_{T^{\prime}}\right)$ is order isomorphic to the space $L^{1}\left(\left|m_{T^{\prime}}\right|\right)([\mathbf{8 2}$, Th. 1 and 4] or [79, Prop. 3.48]), where $\left|m_{T^{\prime}}\right|$ is the variation measure of $m_{T^{\prime}}$. Since by Theorem 
3.7 the optimal range of $T$ with the $p$-th power requirement for the dual map is the space $L^{p}\left(m_{T^{\prime}}\right)^{\prime}$, we obtain that the optimal range is order isomorphic to $L^{p^{\prime}}\left(\left|m_{T^{\prime}}\right|\right)$.

Observe that in the proof of the previous proposition, the main step is to prove that the integration map associated with the Köthe adjoint of the given operator, is compact. A result due to Rybakov (see [78, Cor. 10]) provide us some examples of this compact integration maps. Notice that compactness of the integration map $I_{m_{T^{\prime}}}$ implies that $L^{1}\left(m_{T^{\prime}}\right)$ is an $L^{1}$-space and so an AL-space ([82, Th. 1 and 4]). Other conditions to obtain that $L^{1}(m)$ is an AL-space can be found in [16].

Corollary 3.13. Let $E$ be a Banach lattice and $F$ be a Banach space, such that $E^{*}$ has order continuous norm. Let $T: E \rightarrow F$ be an operator such that $m_{T^{*}}$ is a vector measure and has $\sigma$-finite variation. Then $I_{m_{T^{*}}}^{(p)}$ is a compact operator for somelevery $1<p \leq \infty$.

Proof. $E^{*}$ is order continuous, then $E$ does not have a lattice subspace isomorphic to $\ell^{1}$ (see e.g. [73, Th. 2.4.14]). By hypothesis $m_{T^{*}}$ has finite $\sigma$-finite variation. Applying [79, Lem. 3.53(iv)] we conclude that $\mathscr{R}\left(m_{T^{*}}\right)$ is relatively compact, now [79, Prop. 3.56(I) (iii)] gives the result.

3.2.2. The optimal range for the Volterra operator. The spaces of $p$-integrable functions with respect to the Volterra measure (i.e. the one defined by the Volterra operator) are nowadays well known. The reader can find information about in [79, Ex. 3.76]. It provides the optimal domain space for this operator. In this subsection we analyze the structure of the optimal range for this operator.

Example 3.14 (Volterra operator). Let $V: L^{p}[0,1] \rightarrow L^{q}[0,1]$ be the Volterra operator for $1<q \leq p<2$ which adjoint operator is $r$-th power factorable, $r \geq 1$. Note that $V=H_{0}$ in the Example 3.4, so this condition holds for $r<q^{\prime}$. From Theorem 3.7 we have the following factorization diagram

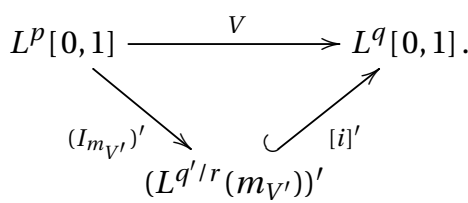

DIAGRAM 3.4. Optimal range for the Volterra operator

Let $\mu$ be Lebesgue measure in $[0,1]$ and denote by $v$ the measure $v:=\left\langle\chi_{[0,1]}, m_{V^{\prime}}\right\rangle$. This measure $v$ is given by

$$
\begin{aligned}
v(A) & :=\left\langle\chi_{[0,1]}, V^{\prime}\left(\chi_{A}\right)\right\rangle=\int_{0}^{1} \chi_{[0,1]}(y) \int_{0}^{1} \chi_{[0, x]}(y) \chi_{A}(x) d \mu(x) d \mu(y) \\
& =\int_{0}^{1} \int_{0}^{1} \chi_{[y, 1]}(x) \chi_{A}(x) d \mu(x) d \mu(y)=\int_{0}^{1} \mu([x, 1] \cap A) d \mu(x), \quad A \in \Sigma .
\end{aligned}
$$


Obviously $v$ is a Rybakov measure $v$ for $m_{V^{\prime}}$ defined by the element $\chi_{[0,1]} \in L^{p}[0,1]$. (See Example 6.46 in [79] for the corresponding Rybakov measure for the case of the Volterra operator). We denote by $h$ the Radon-Nikodým derivative $\frac{d v}{d \mu}$. Observe that by Remark 3.2 we have that $h(x)=\left(V\left(\chi_{[0,1]}\right)\right)(x)=\int_{0}^{x} d y=x$, since the Volterra operator is $\mu$-determined (see [79, Ex. 4.9]). Recall that $L^{q^{\prime} / r}\left(m_{V^{\prime}}\right)$ is a Banach function space over the measure $v$ (and so is $\left.\left(L^{q^{\prime} / r}\left(m_{V^{\prime}}\right)\right)^{\prime}\right)$. The measure $v$ has the same null sets as $\mu$. Then, as we pointed out in Remark 3.3, $[i]^{\prime}$ is given by $[i]^{\prime}(g)(x):=h(x) \cdot g(x) \in L^{q}[0,1]$, where $g \in\left(L^{q^{\prime} / r}\left(m_{V^{\prime}}\right)\right)^{\prime}$ and $x \in[0,1]$. This allows to write the inclusions

$$
V\left(L^{p}[0,1]\right) \subseteq M_{h}\left(\left(L^{q^{\prime} / r}\left(m_{V^{\prime}}\right)\right)^{\prime}\right) \subseteq L^{q}[0,1],
$$

and $\left(L^{q^{\prime} / r}\left(m_{V^{\prime}}\right)\right)^{\prime}$ is the optimal range space, in the sense that was explained. Let us give more information about this space.

We know that $\left(L^{q^{\prime} / r}\left(m_{V^{\prime}}\right)\right)^{\prime}$ is $\left(q^{\prime} / r\right)^{\prime}$-concave, since $L^{q^{\prime} / r}\left(m_{V^{\prime}}\right)$ is $q^{\prime} / r$-convex (see [79, Ch.2]). Now we assume that $r \geq 1$ satisfies that $\left(q^{\prime} / r\right)^{\prime} \leq p$. On one hand, taking into account that the integration map associated with the Volterra operator is again given by the same kernel, and the adjoint map is given by the dual kernel of the Volterra kernel we have that $\left(I_{m_{V^{\prime}}}\right)^{\prime}$ is positive. On the other hand, since $L^{p}[0,1]$ is $p$-convex and thus $\left(q^{\prime} / r\right)^{\prime}$-convex, we have that $\left(I_{m_{V^{\prime}}}\right)^{\prime}$ is $\left(q^{\prime} / r\right)^{\prime}$-convex (see [66, Th. 1.d.9] and comments below it). Using the instance of the Maurey-Rosenthal Theorem 1.3, we have the following factorization diagram

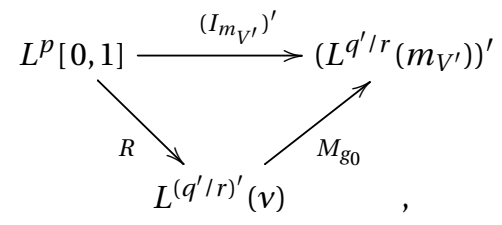

DIAGRAM 3.5. Factorization through an $L^{S}$-space for the adjoint of the integration map $\left(I_{m_{V^{\prime}}}\right)^{\prime}$

where $R$ is a continuous operator and $0<g_{0} \in\left(L^{q^{\prime} / r}\left(m_{V^{\prime}}\right)\right)^{q^{\prime} / r}$ (seeing this space over the control measure $v$, see [12, Lem. 3.7]). Therefore,

$$
V\left(L^{p}[0,1]\right) \subseteq M_{h \cdot g_{0}}\left(R\left(L^{p}[0,1]\right)\right) \subseteq M_{h}\left(\left(L^{q^{\prime} / r}\left(m_{V^{\prime}}\right)\right)^{\prime}\right),
$$

and $M_{h}\left(\left(L^{q^{\prime} / r}\left(m_{V^{\prime}}\right)\right)^{\prime}\right)$ is the optimal range satisfying the $r$-th power factorability requirement on the adjoint operator. 

CHAPTER 4

\section{$(p, q)$-TH POWER FACTORABLE OPERATORS}

In this chapter we study the class of $(p, q)$-th power factorable operators. They are characterized by a combination of Theorem 3.7 and Theorem 1.7 on optimal domains via $p$-th power spaces. The main result in this chapter is Theorem 4.7 , that gives characterizations of $(p, q)$-th power factorable operators by means of general different factorization schemes.

\subsection{The $(p, q)$-th power factorable operator}

A $p$-th power factorable operator can be easily characterized by means of a factorization diagram, as has been shown in [79, Lem. 5.3] for the case of a $\mu$-determined operator or in Proposition 1.8 for a more general case. In this chapter we provide a square factorization scheme for a new class of $p$-th power factorable operators with $q$-th power factorable adjoint. For the sake of clarity all along in this chapter we will write the results for operators between Banach function spaces over the same measure. However, these results work also for Banach function spaces over different measures.

Definition 4.1. Let $1 \leq p, q<\infty$. Let $X$ and $Y$ be two Banach function spaces based on finite measures such that $X$ and $Y^{\prime}$ are order continuous. We say that an operator $T: X \rightarrow$ $Y$ is $(p, q)$-th power factorable if there exist a Banach space $E$ and operators $R: X \rightarrow E$ and $S: E \rightarrow Y$ such that $T=S \circ R, R$ is $p$-th power factorable and the Köthe adjoint operator of $S, S^{\prime}: Y^{\prime} \rightarrow E^{*}$ is $q$-th power factorable.

Notice that from the Definition 1.6 of $p$-th power factorable operator, the spaces $X$ and $Y^{\prime}$ are implicitly assumed order continuous, however for the aim of clarity we include these requirements in this definition. Now let us show a relevant example of this class of operators.

Example 4.2 (Hardy type operator). Let $s \geq 0$ and consider the kernel operator $H_{s}$ with kernel function $K(x, y):=\frac{1}{x^{s}} \chi_{[0, x]}(y)$, i.e.

$$
\left(H_{s} f\right)(x)=\int_{0}^{1} K(x, y) f(y) d y=\int_{0}^{1} \frac{1}{x^{s}} f(y) \chi_{[0, x]}(y) d y=\frac{1}{x^{s}} \int_{0}^{x} f(y) d y .
$$

Note that by Hölder-Rogers inequality the operator $H_{s}: L^{u}[0,1] \rightarrow L^{v}[0,1]$ is always well defined and continuous for $1 \leq v<u$ when $s<\frac{1}{v}-\frac{1}{u}<1$ (in fact, it is continuous in more 
cases, see for instance [8, Ch. 3 Th. 3.10]). Under these restrictions for $u, v$ and $s$ we can consider the following factorization. For $h(x)=x^{-s} \in L^{u}[0,1]^{L^{v}[0,1]}$ and the Volterra operator $V: L^{u}[0,1] \rightarrow L^{u}[0,1]$, we can write

$$
H_{s}=M_{h} \circ V: L^{u}[0,1] \stackrel{V}{\longrightarrow} L^{u}[0,1] \stackrel{M_{h}}{\longrightarrow} L^{v}[0,1] .
$$

It is known that $V$ is $p$-th power factorable for all $1 \leq p \leq u$ (see [79, Ex. 5.9]). On the other hand, observe that $\left(M_{h}\right)^{\prime}=M_{h}: L^{v^{\prime}}[0,1] \rightarrow L^{u^{\prime}}[0,1]$ and for $g \in L^{u}[0,1]$ we have that $M_{h}(f) \in L^{\nu}[0,1]$. Take then an index $1<q \leq v^{\prime}$ such that $s<\frac{1}{u^{\prime}}-\frac{q}{v^{\prime}}<\frac{1}{u^{\prime}}-\frac{1}{v^{\prime}}=\frac{1}{v}-\frac{1}{u}$ (so these requirements are compatible with the restrictions on the indexes written above). Then a direct computation using Hölder-Rogers inequality gives the continuity of the map $M_{h}: L^{v^{\prime} / q} \rightarrow L^{u^{\prime}}$, i.e. $\left(M_{h}\right)^{\prime}$ is $q$-th power factorable. Consequently, $H_{s}$ is $(p, q)$-th power factorable for $1 \leq p \leq u$ and $1 \leq q<v^{\prime}\left(\frac{1}{u^{\prime}}-s\right)$.

Remark 4.3. Note that the assumption on the $p$-th power factorability of a non trivial operator $T: X \rightarrow E$ together with the order continuity of $X$ implies that $\left(X_{[p]}\right)^{*}$ is nontrivial. Certainly, the composition of the extension $T_{[p]}$ of $T$ with a functional $x^{*} \in E^{*}$ produces a continuous functional $z^{*}$ on the quasi-normed space $\left(X_{[p]},\|\cdot\|_{X_{[p]}}\right)$. Let us see this with a commutative diagram

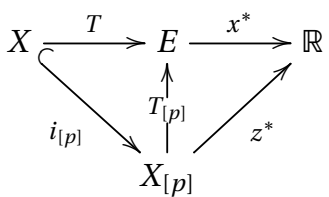

Diagram 4.1. Duality for the $p$-th power space

Observe that the dual spaces $\left(X_{[p]}\right)^{\prime}$ and $\left(\left(Y^{\prime}\right)_{[q]}\right)^{\prime}$ of the quasi-Banach function spaces $X_{[p]}$ and $\left(Y^{\prime}\right)_{[q]}$ are non-empty. These last spaces appear implicitly in the Definition 4.1.

4.1.1. Characterizations for $(p, q)$-th power factorable operators. The following result gives the a first characterization for $(p, q)$-th power factorable operators. Hereafter we use the following notation for inclusion and inclusion/quotient maps: $i_{[p]}: X \hookrightarrow X_{[p]}$, $j_{[q]}: Y^{\prime} \hookrightarrow\left(Y^{\prime}\right)_{[q]}$ and, when $T$ is $(p, q)$-th power factorable, $X \hookrightarrow_{[i]} L^{p}\left(m_{R}\right), Y^{\prime} \hookrightarrow_{[j]} L^{q}\left(m_{S^{\prime}}\right)$, where $T=S \circ R, R$ is $p$-th power factorable and $S^{\prime}$ is $q$-th power factorable.

Theorem 4.4. Let $1 \leq p, q<\infty$. Let $X$ and $Y$ be Banach function spaces such that $X$ and $Y^{\prime}$ are order continuous and $Y$ is Fatou. Let $T: X \rightarrow Y$ be an operator. Then $T$ is $(p, q)$-th power factorable if and only if there exists an operator

$$
T_{[p, q]}: X_{[p]} \rightarrow\left(\left(Y^{\prime}\right)_{[q]}\right)^{\prime}
$$

such that $T=\left(j_{[q]}\right)^{\prime} \circ T_{[p, q]} \circ i_{[p]}$

Proof. Assume that there exists $T_{[p, q]}: X_{[p]} \rightarrow\left(\left(Y^{\prime}\right)_{[q]}\right)^{\prime}$ such that $T=\left(j_{[q]}\right)^{\prime} \circ T_{[p, q]}$ 。 $i_{[p]}$, where $i_{[p]}: X \hookrightarrow X_{[p]}$ and $j_{[q]}: Y^{\prime} \hookrightarrow\left(Y^{\prime}\right)_{[q]}$ denote the inclusions. Then, by definition, 
$T_{[p, q]} \circ i_{[p]}$ is $p$-th power factorable and $\left(\left(Y^{\prime}\right)_{[q]}\right)^{\prime}$ is a Banach space since it is the dual of a quasi-Banach space (see [55, p. 1102]). Directly we have that $\left(Y^{\prime}\right)_{[q]} \subseteq\left(\left(Y^{\prime}\right)_{[q]}\right)^{\prime \prime}$. Moreover $Y^{\prime}$ is order continuous. Therefore, $\left(j_{[q]}\right)^{\prime \prime}: Y^{\prime} \rightarrow\left(Y_{[q]}^{\prime}\right)^{\prime \prime}$ factors through the inclusion map $Y^{\prime} \hookrightarrow\left(Y^{\prime}\right)_{[q]}$, and so it is $q$-th power factorable.

For the converse, suppose that $T$ is $(p, q)$-th power factorable. Then consider $E$ the Banach space, so that $T=S \circ R$ where $R: X \rightarrow E$ is a $p$-th power factorable operator and $S: E \rightarrow Y$ is such that $S^{\prime}$ is $q$-th power factorable. Then, by definition we have an extension as

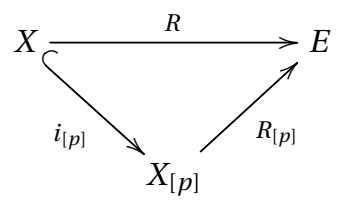

On the other hand, we have a factorization for $S^{\prime}$ as

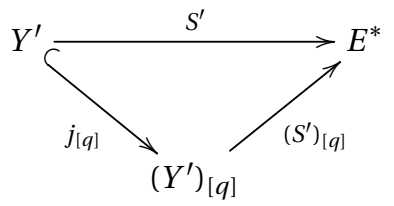

By dualizing and taking into account the properties of the spaces involved, we can obtain the following factorization scheme.

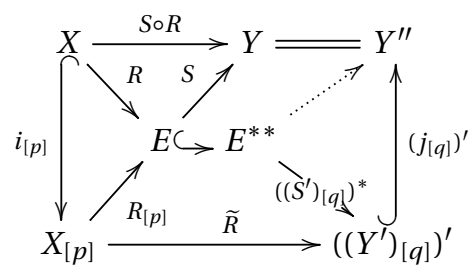

Diagram 4.2. Characterization I for $(p, q)$-th power factorable operators

where $\widetilde{R}=\left(\left(S^{\prime}\right)_{[q]}\right)^{*} \circ R_{[p]}$ plays the role of $T_{[p, q]}$. Since $\left(j_{[q]}\right)^{\prime \prime}=j_{[q]}$, we got the result.

This scheme of factorization allows us to obtain the following result about the Köthe adjoint of a $(p, q)$-th power factorable operator.

Corollary 4.5. Let $1 \leq p, q<\infty$. Let $X$ and $Y$ be Banach function spaces such that $X$ and $Y^{\prime}$ are order continuous and $X$ and $Y$ are Fatou. Let $T: X \rightarrow Y$ be $a(p, q)$-th power factorable operator. Then the Köthe adjoint $T^{\prime}: Y^{\prime} \rightarrow X^{\prime}$ is $(q, p)$-th power factorable.

Proof. Thanks to Theorem 4.4, there is an operator $T_{[p, q]}: X_{[p]} \rightarrow\left(\left(Y^{\prime}\right)_{[q]}\right)^{\prime}$. Thus we consider the following restriction $\left.\left(T_{[p, q]}\right)^{\prime}\right|_{\left(Y^{\prime}\right)_{[q]}}:\left(Y^{\prime}\right)_{[q]} \hookrightarrow\left(\left(Y^{\prime}\right)_{[q]}\right)^{\prime \prime} \rightarrow\left(X_{[p]}\right)^{\prime}=\left(\left(X^{\prime \prime}\right)_{[p]}\right)^{\prime}$, since $X$ is Fatou. Moreover $X^{\prime \prime}=X$ is order continuous and so is $Y^{\prime}$. So, applying again Theorem 4.4 to $T^{\prime}: Y^{\prime} \rightarrow X^{\prime}$ we have that $T^{\prime}$ is $(q, p)$-th power factorable. 
Example 4.6. Classical factorization theorems provide easy examples of $(1,1)$-th power factorable operators. Suppose that we have a weakly compact operator $T: X \rightarrow Y$, where $X$ and $Y^{\prime}$ are order continuous. Thus $T$ factors through a reflexive Banach space (see e.g. [31, Cor. VIII.4.9]), we can find a factorization for $T: X \stackrel{R}{\rightarrow} F \stackrel{S}{\rightarrow} Y$, where $F$ is a reflexive Banach space. In particular, $R$ can be extended to its optimal domain $L^{1}\left(m_{R}\right)$ and the same holds for $S^{\prime}: Y^{\prime} \rightarrow F^{*}$ to the corresponding space $L^{1}\left(m_{S^{\prime}}\right)$. Consequently, $T$ is $(1,1)$ th power factorable, and the factorization space is reflexive.

The following theorem gives a factorization for $(p, q)$-th power factorable operators involving spaces $L^{p}(m)$ for a vector measure $m$. Roughly speaking they are a class of operators which allow factorization diagrams through an operator between $L^{p}\left(m_{1}\right)$ and $L^{q}\left(m_{2}\right)^{\prime}$. Observe that, if $m_{1}$ and $m_{2}$ are scalar measures and $q^{\prime} \leq p$, this setting gives well known square factorization schemes for operators between Banach function spaces satisfying certain domination assumptions of concavity type for the associated bilinear form (see [25, Th. 3.2], see also [23, Th. 2]).

Theorem 4.7. Let $1 \leq p, q<\infty$. Let $X$ and $Y$ be two Banach function spaces over the same finite measure, so that $X$ and $Y^{\prime}$ are order continuous and $Y$ is Fatou. Let $T: X \rightarrow Y$ be an operator. Then the following statements are equivalent:

(1) $T$ is $(p, q)$-th power factorable.

(2) There exists some $K>0$ such that

$$
|\langle T(f), g\rangle| \leq K\|f\|_{X_{[p]}}\|g\|_{\left(Y^{\prime}\right)[q]}, \quad f \in X, g \in Y^{\prime} .
$$

(3) There exists an operator $T_{[p, q]}: X_{[p]} \rightarrow\left(Y_{[q]}^{\prime}\right)^{\prime}$ such that the following diagram is commutes

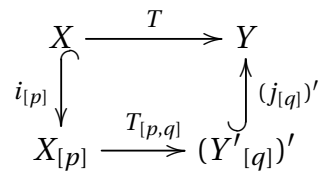

(4) There exist two operators $F: X \rightarrow\left(\left(Y^{\prime}\right)_{[q]}\right)^{\prime}$ and $G: Y^{\prime} \rightarrow\left(Y^{\prime}\right)_{[q]}$ and an operator $H: L^{p}\left(m_{F}\right) \rightarrow\left(L^{q}\left(m_{G^{\prime}}\right)\right)^{\prime}$ such that the following diagram commutes

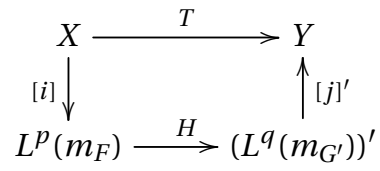

(5) There exist an operator $H, a$ Banach space $E$ and two operators $R: X \rightarrow E$ and $S: E \rightarrow Y$ such that $T=S \circ R$ such that the following diagram commutes

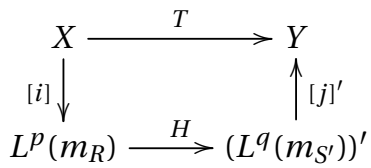

DiAGRAM 4.3. Characterization II for $(p, q)$-th power factorable operators 
Proof. (1) $\Leftrightarrow$ (3) was proved in Theorem 4.4. For (2) $\Rightarrow$ (3) we will prove that the operator $T$ is $\left(\|\cdot\|_{X_{[p]}}-\|\cdot\|_{\left.\left(\left(Y^{\prime}\right)_{[q]}\right)^{\prime}\right)}\right.$-continuous. Let $s$ be a simple function. By hypothesis we have that $|\langle T(f), s\rangle| \leq K\|f\|_{X_{[p]}}\|s\|_{\left(Y^{\prime}\right)[q]}$, for all $f \in X$. In consequence, for all simple function $s \in B_{\left(Y^{\prime}\right)_{[q]}}$ we have that $|\langle T(f), s\rangle| \leq K\|f\|_{X_{[p]}}$, i.e.

$$
\sup _{s}|\langle T(f), s\rangle| \leq K\|f\|_{X_{[p]}},
$$

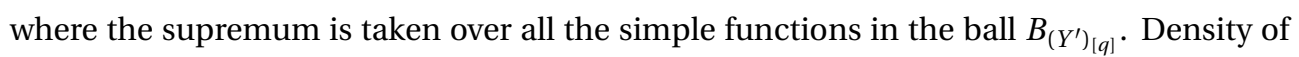
simple functions in $\left(Y^{\prime}\right)_{[q]}$, since $Y^{\prime}$ is order continuous, and the continuity of $T$ allows us to extend this inequality to all $g \in B_{\left(Y^{\prime}\right)_{[q]}}$, hence we obtain for every $f \in X$,

$$
\|T f\|_{\left(\left(Y^{\prime}\right)_{[q]}\right)^{\prime}}=\sup _{g \in B_{\left(Y^{\prime}\right)}[q]}|\langle T(f), g\rangle| \leq K\|f\|_{X_{[p]}} .
$$

$T(X) \subseteq\left(\left(Y^{\prime}\right)_{[q]}\right)^{\prime}$, then we can extend $T$ to $X_{[p]}$ since $X$ is dense in $X_{[p]}$ and $T$ is continuous. In other words, there exists $T_{[p, q]}: X_{[p]} \rightarrow\left(\left(Y^{\prime}\right)_{[q]}\right)^{\prime}$ such that $T=\left(j_{[q]}\right)^{\prime} \circ T_{[p, q]} \circ i_{[p]}$ where $i_{[p]}$ denotes the inclusion of $X$ into $X_{[p]}$ and $j_{[q]}$ the inclusion of $Y^{\prime}$ into $\left(Y^{\prime}\right)_{[q]}$.

(3) $\Rightarrow$ (2) Let $f \in X$ and $g \in Y^{\prime}$. Then

$$
\begin{aligned}
|\langle T(f), g\rangle| & =\left|\left\langle\left(j_{[q]}\right)^{\prime} \circ T_{[p, q]} \circ i_{[p]}(f), g\right\rangle\right|=\left|\left\langle T_{[p, q]} \circ i_{[p]}(f),\left(j_{[q]}\right)^{\prime \prime}(g)\right\rangle\right| \\
& \leq\left\|T_{[p, q]}\right\|\left\|i_{[p]}(f)\right\|_{X_{[p]}}\left\|\left(j_{[q]}\right)^{\prime \prime}(g)\right\|_{\left(Y_{[q]}^{\prime}\right)^{\prime \prime}} \leq K\|f\|_{X_{[p]}}\|g\|_{Y_{[q]}^{\prime}},
\end{aligned}
$$

where $K=\left\|T_{[p, q]}\right\|\left\|i_{[p]}\right\|\left\|\left(j_{[q]}\right)^{\prime \prime}\right\|$.

(3) $\Rightarrow$ (4) Let us define the Banach space $E:=\left(\left(Y^{\prime}\right)_{[q]}\right)^{\prime}$ (see e.g. [55, p. 1102]). Consider the $p$-th power factorable map $F:=T_{[p, q]} \circ i_{[p]}: X \rightarrow E$ and the $q$-th power factorable map $G:=j_{[q]}$ given by the inclusion of $Y^{\prime}$ into $\left(Y^{\prime}\right)_{[q]}$. Using the order continuity and Fatou properties of the involved spaces and the characterization given in Proposition 1.8 we obtain the factorization

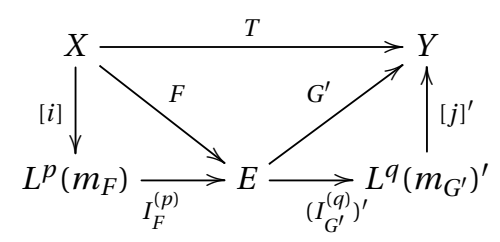

where $H:=\left(I_{G^{\prime}}^{(q)}\right)^{\prime} \circ I_{F}^{(p)}$.

Note that the proof of the previous implication gives in fact also (3) $\Rightarrow$ (5), for $E=$ $\left(\left(Y^{\prime}\right)_{[q]}\right)^{\prime}$ and $S=G^{\prime}$. (4) $\Rightarrow(5)$ is evident. For (5) $\Rightarrow(1)$, by hypothesis $X \hookrightarrow_{[i]} L^{p}\left(m_{R}\right)$ and also $Y^{\prime} \hookrightarrow_{[j]} L^{q}\left(m_{S^{\prime}}\right)$. By Proposition 1.8 we have that $R$ is $p$-th power factorable and $S^{\prime}$ is $q$-th power factorable and so the result is obtained.

Remark 4.8. Let $1 \leq p, q<\infty$ be such that $q^{\prime}<p$. Let $T: X \rightarrow Y$ be a positive $(p, q)$-th power factorable operator. In virtue of the Maurey-Rosenthal Theorems 1.3 and 1.4 we always have the following scheme 


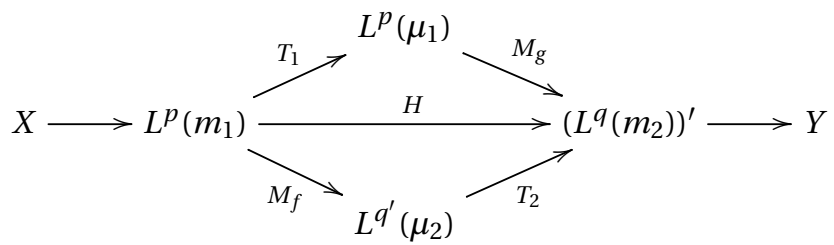

DiAgram 4.4. Maurey-Rosenthal factorization applied to a $(p, q)$-th power factorable operator

where $f \in X^{q}$ and $g \in\left(Y^{\prime}\right)^{p^{\prime}}, \mu_{i}$ are scalar measures, $m_{i}$ are vector measures and $T_{i}$ are operators, for $i=1,2$. This provides a first estimation for the optimal domain and the optimal range, namely $M_{f}\left(L^{p}\left(m_{1}\right)\right) \subseteq L^{q^{\prime}}\left(\mu_{2}\right)$ and $M_{g}\left(L^{p}\left(\mu_{1}\right)\right) \subseteq\left(L^{q}\left(m_{2}\right)\right)^{\prime}$.

Remark 4.9. Under the hypothesis of Theorem 4.7, let $T: X \rightarrow Y$ be an operator. A necessary condition for $(p, q)$-th power factorability is that $T$ to be $p$-th power factorable and $T^{\prime}$ to be $q$-th power factorable. This follows immediately from [79, Lem. 5.4], since $T=S \circ R$, thus $T_{[p]}=S \circ R_{[p]}$ and $\left(T^{\prime}\right)_{[q]}=R^{*} \circ\left(S^{\prime}\right)_{[q]}$.

4.1.2. Another related result. In the definition of $(p, q)$-th power factorable operator (Definition 4.1) we need a previous factorization of the operator. If we do not have such prior factorization, we have another condition in order to obtain a square factorization.

Proposition 4.10. Let $1<p, q<\infty$. Let $X$ and $Y$ be Banach function spaces over the finite measure $\mu$, such that $X$ and $Y^{\prime}$ are order continuous and $Y$ is Fatou. Let $T: X \rightarrow Y$ be a $\mu$-determined operator such that $T^{\prime}$ is also $\mu$-determined. Then the following statements are equivalent:

(1) There exists $K>0$ such that

$$
|\langle T(f), g\rangle| \leq K\|f\|_{L^{p}\left(m_{T}\right)}\|g\|_{L^{q}\left(m_{T^{\prime}}\right)}, \quad f \in X, g \in Y^{\prime} .
$$

(2) There exists an operator $I^{(p, q)}: L^{p}\left(m_{T}\right) \rightarrow\left(L^{q}\left(m_{T^{\prime}}\right)\right)^{\prime}$ such that the following diagram is commutative

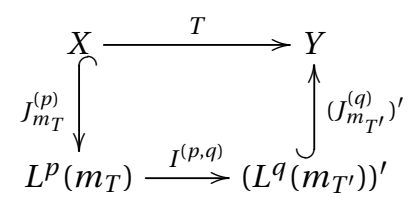

DiAGRAM 4.5. Square factorization through $L^{p}\left(m_{T}\right)$ and $L^{q}\left(m_{T^{\prime}}\right)^{\prime}$ 
Proof. (1) $\Rightarrow$ (2). Let $s$ be a simple function. By hypothesis we have that $|\langle T(f), s\rangle| \leq$ $K\|f\|_{L^{p}\left(m_{T}\right)}\|s\|_{L^{q}\left(m_{T^{\prime}}\right)}$, for all $f \in X$. In consequence, for all simple functions $s \in B_{L^{q}\left(m_{T^{\prime}}\right)}$ we have that $|\langle T(f), s\rangle| \leq K\|f\|_{L^{p}\left(m_{T}\right)}$, i.e. $\sup _{s}|\langle T(f), s\rangle| \leq K\|f\|_{L^{p}\left(m_{T}\right)}$, where the supremum is taken over all the simple functions in the ball $B_{L^{q}\left(m_{T^{\prime}}\right)}$. Density of simple functions in $L^{q}\left(m_{T^{\prime}}\right)$, since it is order continuous, and the continuity of $T$ allows us to extend this inequality to all $g \in B_{L^{q}\left(m_{T^{\prime}}\right)}$, hence we obtain

$$
\|T f\|_{\left(L^{q}\left(m_{T^{\prime}}\right)^{\prime}\right.}=\sup _{g \in B_{L} q_{\left(m_{T^{\prime}}\right)}}|\langle T(f), g\rangle| \leq K\|f\|_{L^{p}\left(m_{T}\right)} .
$$

Then we extend $T$ to $L^{p}\left(m_{T}\right)$ and $T(X) \subseteq\left(L^{q}\left(m_{T^{\prime}}\right)\right)^{\prime}$. In other words, there exists an operator $I^{(p, q)}: L^{p}\left(m_{T}\right) \rightarrow\left(L^{q}\left(m_{T^{\prime}}\right)\right)^{\prime}$ such that $T=\left(J_{m_{T^{\prime}}}{ }^{(q)}\right)^{\prime} \circ I^{(p, q)} \circ J_{m_{T}}{ }^{(p)}$ where $J_{m_{T}}$ denotes the inclusion of $X$ into $L^{p}\left(m_{T}\right)$ and $J_{m_{T^{\prime}}}$ the inclusion of $Y^{\prime}$ into $L^{q}\left(m_{T^{\prime}}\right)$, recall that both $T$ and $T^{\prime}$ are $\mu$-determined.

(2) $\Rightarrow$ (1). An standard argument for $T=\left(J_{m_{T^{\prime}}}^{(q)} \circ I^{\prime}(p, q) \circ J_{m_{T}}^{(p)}\right.$, by applying the continuity of the operators involved and the inclusion $L^{q}\left(m_{T^{\prime}}\right) \subseteq L^{q}\left(m_{T^{\prime}}\right)^{\prime \prime}$ we obtain the result, i.e.

$$
|\langle T(f), g\rangle| \leq\left(C\left\|I^{(p, q)}\right\|\left\|J_{m_{T}}^{(p)}\right\|\left\|\left(J_{m_{T^{\prime}}}^{(q)}\right){ }^{\prime \prime}\right\|\right)\|f\|_{L^{p}\left(m_{T}\right)}\|g\|_{L^{q}\left(m_{T^{\prime}}\right)},
$$

for every $f \in X$ and every $g \in Y^{\prime}$.

Remark 4.11. Observe that in the proof of the implication (2) $\Rightarrow(1)$ of the previous proposition, one can remove the requirements of $\mu$-determination.

The following example presents a class of kernel operators that always satisfies the condition (4.2) of Proposition 4.10.

Example 4.12. Let us consider the set $[0,1]$, as usual with the Lebesgue measure, which we will denote by $d x$ and $d y$ for two different variables. So $d x \otimes d y$ is the (Lebesgue) product measure for the product set $[0,1] \times[0,1]$. Let $\eta: \Sigma \otimes \Sigma \rightarrow[0, \infty)$ be a probability measure such that $\eta \ll d x \otimes d y$. Thanks to the Radon-Nikodým Theorem there exists a function $K_{\eta} \in L^{1}(d x \otimes d y)$ such that $\eta(A)=\int_{A} K_{\eta}(x, y) d x \otimes d y$, for every measurable set $A \subseteq[0,1]$, i.e. $d \eta=K_{\eta} d x \otimes d y$. This function $K_{\eta}: \Omega \times \Omega \rightarrow[0, \infty)$ is a positive kernel function. Let $1 \leq p, q, s<\infty$ be such $\frac{1}{s}=\frac{1}{p}+\frac{1}{q}$. Assume that $T_{\eta}: X[0,1] \rightarrow Y[0,1]$ is the kernel operator associated with $K_{\eta}$, so that it is $p$-th power factorable and its adjoint is $q$-th power factorable. Let us define the function $(g \otimes f)(x, y):=g(x) f(y)$, then $g \otimes f=\left(g \otimes \chi_{[0,1]}\right)\left(\chi_{[0,1]} \otimes f\right)$. Let $f \in X(d x)$ and $g \in(Y(d x))^{\prime}$. Applying Hölder-Rogers inequality, Fubini's Theorem, Proposition 1.1(1), the conditions on power factorability and (1.6), we obtain

$$
\begin{aligned}
& \left|\left\langle T_{\eta}(f), g\right\rangle\right|=\int_{[0,1] \times[0,1]}|g \otimes f| K_{\eta} d x \otimes d y \leq C\left(\int_{[0,1] \times[0,1]}|g \otimes f|^{s} d \eta\right)^{1 / s} \\
& \leq C\left(\int_{[0,1] \times[0,1]}\left|\chi_{[0,1]} \otimes f\right|^{p} K_{\eta}(x, y) d x \otimes d y\right)^{1 / p}\left(\int_{[0,1] \times[0,1]}\left|g \otimes \chi_{[0,1]}\right|^{q} K_{\eta}(x, y) d x \otimes d y\right)^{1 / q} \\
& \leq C\left(\int_{0}^{1} \chi_{[0,1]}\left(\int_{0}^{1}|f(y)|^{p} K_{\eta}(x, y) d y\right) d x\right)^{1 / p}\left(\int_{0}^{1} \chi_{[0,1]}\left(\int_{0}^{1}|g(x)|^{q} K_{\eta}(x, y) d x\right) d y\right)^{1 / q} .
\end{aligned}
$$


So following this computation we can conclude that

$$
\begin{aligned}
\left|\left\langle T_{\eta}(f), g\right\rangle\right| & =C\left(\int_{0}^{1}\left(T_{\eta}\right)_{[p]}\left(|f|^{p}\right) d x\right)^{1 / p}\left(\int_{0}^{1}\left(T_{\eta}^{\prime}\right)_{[q]}\left(|g|^{q}\right) d y\right)^{1 / q} \\
& =C\left\|\left(T_{\eta}\right)_{[p]}\left(|f|^{p}\right)\right\|_{L^{1}(d x)}^{1 / p}\left\|\left(T_{\eta}^{\prime}\right)_{[q]}\left(|g|^{q}\right)\right\|_{L^{1}(d y)}^{1 / q} \\
& =C D\left\|\left(T_{\eta}\right)_{[p]}\left(|f|^{p}\right)\right\|_{X(d x)}^{1 / p}\left\|\left(T_{\eta}^{\prime}\right)_{[q]}\left(|g|^{q}\right)\right\|_{(Y(d y))^{\prime}}^{1 / q} \\
& =C D\left\|I_{m_{T_{\eta}}}\left(|f|^{p}\right)\right\|_{X(d x)}^{1 / p}\left\|I_{m_{T_{\eta}^{\prime}}}\left(|g|^{q}\right)\right\|_{(Y(d y))^{\prime}}^{1 / q} \\
& \leq C D\|f\|_{L^{p}\left(m_{T_{\eta}}\right)}\|g\|_{L^{q}\left(m_{T_{\eta}^{\prime}}\right)} \leq C D M\|f\|_{L^{p}\left(m_{T_{\eta}}\right)}\|g\|_{L^{q}\left(m_{T_{\eta}^{\prime}}\right)} .
\end{aligned}
$$

Thus $T_{\eta}$ satisfies (4.2).

\subsection{The non finite measure case}

As a consequence of the definition of $(p, q)$-th power factorable operator, we have been considering finite measure spaces, since otherwise the inclusion $X \subseteq X_{[p]}$ does not make sense. Our construction depends strongly on the finite measure requirement. For example Rybakov measures for vector measures need to be finite, since vector measures on $\sigma$-algebras are always bounded. Otherwise, a vector measure can only be defined on the $\delta$-ring of the measurable sets of finite measure, and the factorization by means of the inclusion/quotient map do not hold (see [13] and references therein for the use of $\delta$-rings in the context of the factorization of operators between Banach function spaces).

However, by means of multipliers that define isometric maps it is possible to extend our results to the case of $\sigma$-finite measures. Let us present a standard construction. Consider a Banach function space $X$ over the $\sigma$-finite measure $\mu$. Then there is a weak order unit $h \in X$. Take a norm one element $0<\varphi \in X^{\prime}$. Let us now define the measure $\bar{\mu}$ by $\bar{\mu}(A):=\int_{A} \varphi h d \mu$, where $A \in \Sigma$. Note that $\bar{\mu}(\Omega)=\int_{\Omega} \varphi h d \mu \leq\|h\|_{X}\|\varphi\|_{X^{\prime}}$, and so $\bar{\mu}$ is finite. Let us define the Banach ideal of classes of $\mu$-measurable functions

$$
X_{h}:=\left\{f \in L^{0}(\mu): f h \in X\right\},
$$

with the lattice norm $\|f\|_{X_{h}}:=\|f h\|_{X}, f \in X_{h}$. Let us see that $X_{h}$ is a Banach function space over $\bar{\mu}$. Clearly, for each $A \in \Sigma,\left\|\chi_{A}\right\|_{X_{h}}=\left\|\chi_{A} h\right\|_{X} \leq\|h\|_{X}<\infty$, and so $\chi_{A} \in X_{h}$. Moreover, each function $f \in X_{h}$ is integrable since

$$
\int_{\Omega}|f| d \bar{\mu} \leq\|f h\|_{X} \cdot\|\varphi\|_{X^{\prime}}=\|f\|_{X_{h}} \cdot\|\varphi\|_{X^{\prime}}<\infty .
$$

This also proves that $X_{h} \hookrightarrow L^{1}(\bar{\mu})$, and so $X_{h}$ is a Banach function space over the finite measure $\bar{\mu}$. Let us also note that the map $f \rightsquigarrow h f$ defines an isometry $X_{h} \rightarrow X$.

Lemma 4.13. Let $X$ and $Y$ be two Banach function spaces over a $\sigma$-finite measure $\mu$. Let $T: X \rightarrow Y$ be an operator. Then there are measurable functions $h$ and $w$ and an operator $T_{0}: X_{h} \rightarrow Y_{w}$ such that $T$ factors through the following scheme 


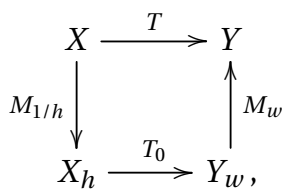

DIAGRAM 4.6. Factorization through Banach function spaces defined over finite measures

where $M_{1 / h}$ and $M_{w}$ are the multipliers associated with $1 / h$ and $w$, respectively. Moreover, $h$ and $w$ can be chosen to be equal and such that $X_{h}$ and $Y_{w}$ are Banach function spaces over the same finite measure $\bar{\mu}$.

Proof. For the proof it suffices to define the operator $T_{0}$ by $T_{0}(g):=1 / w \cdot T(g h), g \in$ $X_{h}$, where $h$ and $w$ are defined as has been explained above. Notice also that if $h$ and $w$ are weak order units for $X$ and $Y$, respectively, the measurable function $h \wedge w$ is also a weak order unit in both spaces. The same can be done by considering $\varphi_{1} \wedge \varphi_{2}$ as the element $\varphi$ appearing in the construction above, where $0<\varphi_{1} \in X^{\prime}$ and $0<\varphi_{2} \in Y^{\prime}$ are the corresponding functions in the definition of the finite measures. So just take $h_{0}:=h \wedge w$, $\varphi:=\varphi_{1} \wedge \varphi_{2}$ and $\bar{\mu}(A):=\int_{A} h_{0} \varphi d \mu, A \in \Sigma$.

Taking into account this result, we can adapt Theorem 4.7 to the non finite measure case as follows.

Corollary 4.14. Let $1 \leq p, q<\infty$. Let $X$ and $Y$ be Banach function spaces over the $\sigma$-finite measure $\mu$. Suppose that $X$ and $Y^{\prime}$ are order continuous and $Y$ is Fatou. Let $T: X \rightarrow Y$ be an operator. Then the following statements are equivalent:

(1) There are a $\mu$-integrable function $h$ and a finite measure $\bar{\mu}$ such that $T$ factors through $a(p, q)$-th power factorable operator $T_{0}: X_{h} \rightarrow Y_{h}$, where $X_{h}$ and $Y_{h}$ are based on $\bar{\mu}$.

(2) There are $a \mu$-integrable function $h$, a finite measure $\bar{\mu}$ and a constant $K$ such that

$$
\left|\left\langle\frac{1}{h} \cdot T(f h), g\right\rangle\right| \leq K\|f\|_{X_{h[p]}}\|g\|_{\left(Y_{h}\right)^{\prime}{ }_{[q]}}, \quad f \in X_{h}, g \in\left(Y_{h}\right)^{\prime},
$$

where $X_{h}$ and $Y_{h}$ are based on $\bar{\mu}$

(3) There are a $\mu$-integrable function $h$, a finite measure $\bar{\mu}$ and $T_{[p, q]}: X_{h[p]} \rightarrow\left(\left(Y_{h}\right)^{\prime}{ }_{[q]}\right)^{\prime}$ an operator, such that the following diagram is commutative

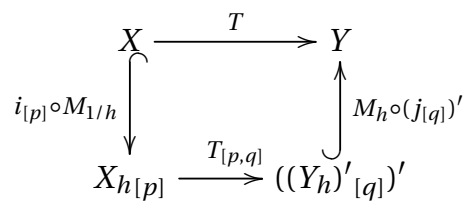

where $X_{h}$ and $Y_{h}$ are based on $\bar{\mu}$. 
(4) There are a $\mu$-integrable function $h$, a finite measure $\bar{\mu}$, an operator $H$, a Banach space $E$ and two operators $R: X_{h} \rightarrow E$ and $S: E \rightarrow Y_{h}$ such that $H=S \circ R$ and $T=\left(M_{h} \circ[j]^{\prime}\right) \circ H \circ\left([i] \circ M_{1 / h}\right)$ and that the following diagram is commutative

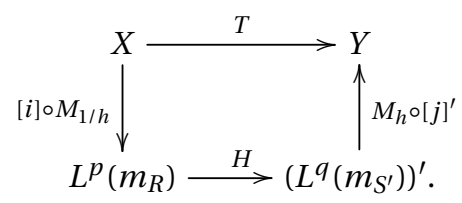

DiAGRAM 4.7. $(p, q)$-th power factorization for non finite measure based spaces

where $X_{h}$ and $Y_{h}$ are based on $\bar{\mu}$.

The proof is a simple consequence of the factorization given in Lemma 4.13 when applied to Theorem 4.7, just taking into account the easily verifiable facts that if $X_{h}$ is a Banach function space based over a finite measure isometric to a Banach function space $X$ based over a $\sigma$-finite measure with the Fatou property, then $X_{h}$ has the Fatou property and the same happens regarding order continuity.

Example 4.15. Take the discrete kernel type operator $T: \ell^{2} \rightarrow \ell^{2}$ given by

$$
T\left(\left(\lambda_{i}\right)\right):=\left(\frac{1}{n 2^{n}} \sum_{i=1}^{n} \lambda_{i}\right)_{n=1}^{\infty} .
$$

Take now $h, \varphi$ and $\bar{\mu}$ in the development above as the ones given by

$$
h:=\left(1 / 2^{i / 2}\right)_{i=1}^{\infty}, \quad \varphi:=\left(1 / 2^{i / 2}\right)_{i=1}^{\infty} \quad \text { and } \quad \bar{\mu}(A):=\sum_{i \in A} 1 / 2^{i}, \quad A \subseteq \mathbb{N} .
$$

Notice that for every $\left(\lambda_{i}\right)_{i=1}^{\infty}$ and $\left(\tau_{i}\right)_{i=1}^{\infty}$, we have the inequality

$$
\left|\sum_{n=1}^{\infty} \frac{2^{n / 2}}{2^{n}}\left(\frac{1}{n 2^{n}} \sum_{i=1}^{n} \frac{\lambda_{i}}{2^{i / 2}}\right) \tau_{n}\right| \leq\left(\sum_{i=1}^{\infty} \frac{\left|\lambda_{i}\right|}{2^{i}}\right) \cdot\left(\sum_{n=1}^{\infty} \frac{\left|\tau_{n}\right|}{2^{n}}\right) .
$$

Consequently, the operator $T$ factors through a $(2,2)$-th power factorable operator. 


\section{CHAPTER 5}

\section{APPLICATIONS OF $(p, q)$-TH POWER FACTORABLE OPERATORS}

In order to develop further the theory of $(p, q)$-th power factorable operators we select two subjects to study. So applications to interpolation (Section 5.1) and independently to kernel operators (Section 5.2)are studied in this chapter.

A classical problem in interpolation theory is to study which properties are inherited by some interpolation method. We consider here the first complex interpolation method defined and studied by Calderón [14]. One of the properties inherited by complex interpolation spaces is weak compactness. The classes of $p$-th and $(p, q)$-th power factorable operators are, for $1<p<\infty$, subsets of the ideal of weakly compact operators (see [63, Cor. $4.3])$. In the first part we analize the behavior of our class of operators under the complex interpolation method.

Our second topic in this chapter is the study of $p$-th and $(p, q)$-th power factorability of kernel operators. We provide some criteria — related to the classical Hölder's inequality and to the Hille-Tamarkin operators - for a kernel operator to be $p$-th or $(p, q)$-th power factorable. A few examples of concrete kernel operators are given.

\subsection{Complex interpolation for operators}

In the present section we prove that, under some natural conditions of order continuity and $\mu$-determination of the operator, the $p$-th and $(p, q)$-th power factorization properties are invariant under the complex interpolation method. So in order to apply the complex interpolation method we assume that all the spaces considered are complex. In this case the definitions of $p$-th and $(p, q)$-th power factorable operator (Definitions 1.6 and 4.1 respectively) should be considered for complex Banach function spaces. Observe moreover, that the proof of Theorem 4.4, that has been used in the present section, can be used for the complex case, since the lattice structure used in such proofs is defined by the modulus of the functions.

5.1.1. Definitions and notation. First we give some basic definitions and their first consequences for complex interpolation of operators. See [8, Ch. 3 Sect. 1], [9, Ch. 2] and [66, Sect. 2.g] for main results and terminology about the complex interpolation method. 
A pair $\left(X_{0}, X_{1}\right)$ of Banach spaces is a compatible pair or simply a pair if there is a Hausdorff topological vector space $\mathscr{X}$ where both $X_{1}$ and $X_{0}$ are continuously embedded. A compatible pair $\left(X_{0}, X_{1}\right)$ of Banach spaces is a regular pair if the intersection $X_{0} \cap X_{1}$ is dense in both $X_{0}$ and $X_{1}$. Observe that if $X_{0}$ and $X_{1}$ are order continuous Banach function spaces, then the pair $\left(X_{0}, X_{1}\right)$ is always a compatible and regular pair, since they are continuously embedded into $L^{0}$ and both contains the simple functions that are dense in both $X_{0}$ and $X_{1}$.

It is said that $X$ is an intermediate space of a compatible pair $\left(X_{0}, X_{1}\right)$ if it is continuously embedded between $X_{0} \cap X_{1}$ and $X_{0}+X_{1}$, i.e. $X_{0} \cap X_{1} \subseteq X \subseteq X_{0}+X_{1}$.

Let $\left(X_{0}, X_{1}\right)$ and $\left(Y_{0}, Y_{1}\right)$ be two compatible pairs. We denote by $T:\left(X_{0}, X_{1}\right) \rightarrow\left(Y_{0}, Y_{1}\right)$ a linear operator $T: X_{0}+X_{1} \rightarrow Y_{0}+Y_{1}$ such that the restrictions $T_{0}:=\left.T\right|_{X_{0}}: X_{0} \rightarrow Y_{0}$ and $T_{1}:=\left.T\right|_{X_{1}}: X_{1} \rightarrow Y_{1}$ are continuous. Thus, we call $T$ an admissible operator with respect to such pairs. Conversely, let us note that if $T_{0}: X_{0} \rightarrow Y_{0}$ and $T_{1}: X_{1} \rightarrow Y_{1}$ are continuous operators, then we can define an admissible operator $T: X_{0}+X_{1} \rightarrow Y_{0}+Y_{1}$ by $T\left(f_{0}+f_{1}\right):=$ $T_{0}\left(f_{0}\right)+T_{1}\left(f_{1}\right)$ whenever $T_{0}(f)=T_{1}(f)$ for every $f \in X_{0} \cap X_{1}$ (see [8, Ch. 3 Prop. 1.6]).

For a compatible pair of Banach function spaces $\left(X_{0}, X_{1}\right)$ and $0<\theta<1$, the CalderónLozanowskǐ product space $X_{0}^{1-\theta} X_{1}^{\theta}$ (see $[\mathbf{1 4 , 6 7 ]}$ ) is

$$
X_{0}^{1-\theta} X_{1}^{\theta}:=\left\{f \in L^{0}:|f|=\left|f_{0}\right|^{1-\theta}\left|f_{1}\right|^{\theta} \text {, for some } f_{0} \in X_{0} \text { and } f_{1} \in X_{1}\right\} .
$$

This space is a Banach space when endowed with the norm given by

$$
\|f\|_{X_{0}^{1-\theta} X_{1}^{\theta}}:=\inf \left\{\left\|f_{0}\right\|_{X_{0}}^{1-\theta}\left\|f_{1}\right\|_{X_{1}}^{\theta}:|f| \leq\left|f_{0}\right|^{1-\theta}\left|f_{1}\right|^{\theta}, f_{i} \in X_{i}, i=0,1\right\} .
$$

In general $X_{0}^{1-\theta} X_{1}^{\theta}$ does not have the interpolation property with respect to $\left(X_{0}, X_{1}\right)$, see [67].

However, under generic hypothesis on $\left(X_{0}, X_{1}\right)$, the Calderón-Lozanowskiu product space coincides with the first Calderón complex interpolation space $\left[X_{0}, X_{1}\right]_{[\theta]}$. We only cite here the following properties that relates both spaces for each $0<\theta<1$.

(1) $X_{0} \cap X_{1} \subseteq\left[X_{0}, X_{1}\right]_{[\theta]} \subseteq X_{0}^{1-\theta} X_{1}^{\theta} \subseteq X_{0}+X_{1}$. In addition we have that

$$
\|f\|_{X_{0}^{1-\theta} X_{1}^{\theta}}=\|f\|_{\left[X_{0}, X_{1}\right]_{[\theta]}}
$$

for all $f \in\left[X_{0}, X_{1}\right]_{[\theta]}$.

(2) If one of the spaces $X_{0}$ or $X_{1}$ is order continuous then $X_{0}^{1-\theta} X_{1}^{\theta}$ is also order continuous ([61, Th. 1.14]) and the equality $\left[X_{0}, X_{1}\right]_{[\theta]}=X_{0}^{1-\theta} X_{1}^{\theta}$ holds isometrically.

Recall that if $X$ is a Banach function space over a finite measure space $(\Omega, \Sigma, \mu), E$ is a Banach space and $T: X \rightarrow E$ is an order continuous (and continuous) $\mu$-determined operator, then we have an associated vector measure $m_{T}: \Sigma \rightarrow E$, defined by $m_{T}(A):=T\left(\chi_{A}\right)$, such that $X \subseteq L^{1}\left(m_{T}\right)$ is an injective inclusion and the integration operator, $I_{m_{T}}(f):=$ $\int_{\Omega} f d m_{T}$ for $f \in L^{1}\left(m_{T}\right)$ is an extension of $T$, i.e. $T(f)=I_{m_{T}}(f)$ for every $f \in X$. If $X$ 
is order continuous or has the Fatou property, then every continuous operator $T$ defined on $E$ is order continuous.

Let us consider now a compatible pair of Banach function spaces $\left(X_{0}, X_{1}\right)$ (over the same finite measure), a compatible pair of Banach spaces $\left(E_{0}, E_{1}\right)$ and an admissible operator $T:\left(X_{0}, X_{1}\right) \rightarrow\left(E_{0}, E_{1}\right)$. Observe that if the restrictions $T_{0}: X_{0} \rightarrow E_{0}$ and $T_{1}: X_{1} \rightarrow E_{1}$ are order continuous and $\mu$-determined operators, then for every $0<\theta<1$ the interpolated operator $T_{\theta}:\left[X_{0}, X_{1}\right]_{[\theta]} \rightarrow\left[E_{0}, E_{1}\right]_{[\theta]}$ is order continuous and $\mu$-determined too. Denote by $m_{0}, m_{1}$ and $m_{\theta}$ the vector measures associated with $T_{0}, T_{1}$ and $T_{\theta}$ respectively. Let us note that $m_{0}(A)=m_{1}(A)=m_{\theta}(A)=T\left(\chi_{A}\right)$ for every $A \in \Sigma$ and $0<\theta<1$. That is, the measures $m_{\theta}, 0<\theta<1$ bring the same values as $m_{0}$ and $m_{1}$, and their range is included in $E_{0} \cap E_{1}$. However the associated spaces $L^{p}\left(m_{\theta}\right)$ would not be the same for all measures since the range space $\left[E_{0}, E_{1}\right]_{[\theta]}$ of each measure may differs from each other.

The technique that we will use consists in interpolating the extensions of the $p$-th power factorable operators, then we prove that the domain of the interpolation of these extensions coincides with the extended domain of the interpolation of the original domains.

5.1.2. Interpolation for $p$-th power factorable operators. Let us show that the $p$-th power space of the Calderón-Lozanowskii product is the Calderón-Lozanowskiu product of some $p$-th power spaces. We note that the $p$-th power space of a Banach function space may be a quasi-Banach function space, e.g. $L_{[p]}^{1}=L^{1 / p}(p>1)$.

With the same definitions as given for a pair of Banach function spaces, if we consider a pair $\left(Z_{0}, Z_{1}\right)$ of quasi-Banach function spaces, it is very easy to obtain that, for every $0<\theta<1$, the product space $Z_{0}^{1-\theta} Z_{1}^{\theta}$ is a quasi-Banach space when endowed with the quasi-norm $\|\cdot\|_{Z_{0}^{1-\theta} Z_{1}^{\theta}}$, see $\left[\mathbf{4 4 , 5 4 ]}\right.$. If the quasi-norms of $Z_{0}$ and $Z_{1}$ are continuous then $\|\cdot\|_{Z_{0}^{1-\theta} Z_{1}^{\theta}}$ is also continuous for every $0<\theta<1$. Note that this is the case for a pair $\left(Z_{0}, Z_{1}\right)=\left(X_{0\left[p_{0}\right]}, X_{1\left[p_{1}\right]}\right)$ where each space is a $p$-th power of a Banach space since for every Banach space $X$ and every $0<p<\infty$, the functional $\|\cdot\|_{X_{[p]}}$ is a continuous quasi-norm for which $X_{[p]}$ is complete (see previous comments to the Proposition 1.5). Observe that $\left(X_{0[p]}, X_{1[p]}\right)$ is a compatible pair, since the $p$-th power space is continuously embedded into $L^{0}$. For $0<p<1$, the $p$-th power space $X_{[p]}$ is still a Banach function space. Otherwise, for $1 \leq p<\infty, X_{[p]}$ is a Banach function space, when endowed with $\|\cdot\|_{X_{[p]}}$, if and only if $X$ is $p$-convex with constant one (see [79, Prop. 2.23(i)(ii)]). Given $0<p_{0}, p_{1}<\infty$ and $0<\theta<1$, denote by $p_{\theta}$ and $\widetilde{p_{\theta}}$ the numbers such that

$$
\frac{1}{p_{\theta}}=\frac{1-\theta}{p_{0}}+\frac{\theta}{p_{1}} \quad \text { and } \quad \frac{1}{\widetilde{p_{\theta}}}=\frac{1-\theta}{p_{1}}+\frac{\theta}{p_{0}},
$$

respectively. Hence $\frac{1}{p_{\theta}}+\frac{1}{\overline{p_{\theta}}}=\frac{1}{p_{1}}+\frac{1}{p_{0}}$. 
Lemma 5.1. Let $\left(X_{0}, X_{1}\right)$ be a pair of Banach function spaces. If $0<p, p_{0}, p_{1}<\infty$ and $0<$ $\theta<1$, then

(1) $\left(X_{0}^{1-\theta} X_{1}^{\theta}\right)_{[p]} \equiv\left(X_{0[p]}\right)^{1-\theta}\left(X_{1[p]}\right)^{\theta}$.

(2) $\left(X_{0\left[p_{0}\right]}\right)^{1-\theta}\left(X_{1\left[p_{1}\right]}\right)^{\theta} \equiv\left(X_{0}^{1-\alpha} X_{1}^{\alpha}\right)_{\left[p_{\alpha}\right]}$ where $\alpha:=\frac{\theta p_{1}}{(1-\theta) p_{0}+\theta p_{1}}$.

Proof. (1). Let $f \in\left(X_{0}^{1-\theta} X_{1}^{\theta}\right)_{[p]}$, and let $f_{i} \in\left(X_{i}\right)^{+}, i=0,1$ be such that $|f|^{1 / p} \leq f_{0}^{1-\theta} f_{1}^{\theta}$, and this happens if and only if $|f| \leq\left(f_{0}^{p}\right)^{1-\theta}\left(f_{1}^{p}\right)^{\theta}$ if and only if $|f| \leq g_{0}^{1-\theta} g_{1}^{\theta}$, where for $i=0,1, g_{i}:=f_{i}^{p} \in X_{i[p]}$. So the following equalities hold

$$
\left(\left\|f_{0}\right\|_{X_{0}}^{1-\theta}\left\|f_{1}\right\|_{X_{1}}^{\theta}\right)^{p}=\left(\left\|\left|g_{0}\right|^{1 / p}\right\|_{X_{0}}^{p}\right)^{1-\theta}\left(\left\|\left|g_{1}\right|^{1 / p_{\|}}\right\|_{X_{1}}^{p}\right)^{\theta}=\left\|g_{0}\right\|_{X_{0[p]}}^{1-\theta}\left\|g_{1}\right\|_{X_{1[p]}}^{\theta},
$$

where $|f| \leq\left(f_{0}^{p}\right)^{1-\theta}\left(f_{1}^{p}\right)^{\theta}$ and also $|f| \leq g_{0}^{1-\theta} g_{1}^{\theta}$, for $g_{i}:=f_{i}^{p} \in X_{i[p]}, i=0,1$ Thus, taking the infimum in (5.2) among such values, we obtain

$$
\|f\|_{\left(X_{0}^{1-\theta} X_{1}^{\theta}\right)_{[p]}}=\left\||f|^{1 / p_{\|}}\right\|_{X_{0}^{1-\theta} X_{1}^{\theta}}^{p}=\|f\|_{\left(X_{0[p]}\right)^{1-\theta}\left(X_{1[p]}\right)^{\theta}},
$$

for every $f \in\left(X_{0}^{1-\theta} X_{1}^{\theta}\right)_{[p]}$. Conversely, the same result holds for each $f \in\left(X_{0[p]}\right)^{1-\theta}\left(X_{1[p]}\right)^{\theta}$.

(2). Let us note that for the given $\alpha:=\frac{\theta p_{1}}{(1-\theta) p_{0}+\theta p_{1}}$ we have that $p_{\alpha}=(1-\theta) p_{0}+\theta p_{1}$. That is, we have the following equality

$$
\frac{1}{(1-\theta) p_{0}+\theta p_{1}}=\frac{1-\alpha}{p_{0}}+\frac{\alpha}{p_{1}}
$$

Thus $\alpha p_{\alpha}=\theta p_{1}$ and also $(1-\alpha) p_{\alpha}=(1-\theta) p_{0}$. Obviously, if $p_{0}=p_{1}$ then $\alpha=\theta$ and this is the case considered in the assertion (1).

Let $f \in\left(X_{0\left[p_{0}\right]}\right)^{1-\theta}\left(X_{1\left[p_{1}\right]}\right)^{\theta}$ and let $g_{i} \in\left(X_{i\left[p_{i}\right]}\right)^{+}(i=0,1)$ be such that $|f| \leq g_{0}^{1-\theta} g_{1}^{\theta}$. Observe that $g_{i} \in X_{i\left[p_{i}\right]}$ if and only if $f_{i}:=g_{i}^{1 / p_{i}} \in X_{i}(i=0,1)$, in this case we have that $|f| \leq\left|f_{0}\right|^{(1-\theta) p_{0}}\left|f_{1}\right|^{\theta p_{1}}=\left|f_{0}\right|^{(1-\alpha) p_{\alpha}}\left|f_{1}\right|^{\alpha p_{\alpha}}$, i.e. $|f|^{1 / p_{\alpha}} \leq\left|f_{0}\right|^{(1-\alpha)}\left|f_{1}\right|^{\alpha}$. Then

$$
\begin{aligned}
\left\|g_{0}\right\|_{X_{0\left[p_{0}\right]}}^{1-\theta}\left\|g_{1}\right\|_{X_{1\left[p_{1}\right]}}^{\theta} & =\left(\left\|g_{0}^{1 / p_{0}}\right\|_{X_{0}}^{p_{0}}\right)^{1-\theta}\left(\left\|g_{1}^{1 / p_{1}}\right\|_{X_{1}}^{p_{1}}\right)^{\theta} \\
& =\left\|f_{0}\right\|_{X_{0}}^{(1-\theta) p_{0}}\left\|f_{1}\right\|_{X_{1}}^{\theta p_{1}}=\left\|f_{0}\right\|_{X_{0}}^{(1-\alpha) p_{\alpha}}\left\|f_{1}\right\|_{X_{1}}^{\alpha p_{\alpha}}=\left(\left\|f_{0}\right\|_{X_{0}}^{1-\alpha}\left\|f_{1}\right\|_{X_{1}}^{\alpha}\right)^{p_{\alpha}},
\end{aligned}
$$

where $|f| \leq g_{0}^{1-\theta} g_{1}^{\theta}$ and $|f|^{1 / p_{\alpha}} \leq\left|f_{0}\right|^{(1-\alpha)}\left|f_{1}\right|^{\alpha}$. Finally, taking the infimum we obtain the equality of the quasi-norms. If $f \in\left(X_{0}^{1-\alpha} X_{1}^{\alpha}\right)_{\left[p_{\alpha}\right]}$ we can follow the reverse way.

Several approaches has been considered to extend the complex interpolation method to the setting of quasi-Banach spaces, see for example $[\mathbf{2 2 ,} \mathbf{4 4 , 5 4 , 8 4 , 9 1 , 1 0 2 ] . ~ C o m p l e x ~}$ interpolation of $p$-convex spaces has been considered in [91].

Remark 5.2. Notice that if in a pair $\left(X_{0}, X_{1}\right)$ of Banach function spaces, at least one of the spaces is order continuous then the product spaces $\left(X_{0\left[p_{0}\right]}\right)^{1-\theta}\left(X_{1\left[p_{1}\right]}\right)^{\theta}$ are order continuous for $0<\theta<1$ and we can apply [44, Th. 3.3] to obtain the equality

$$
\left[X_{0\left[p_{0}\right]}, X_{1\left[p_{1}\right]}\right]_{[\theta]}=\left(X_{0\left[p_{0}\right]}\right)^{1-\theta}\left(X_{1\left[p_{1}\right]}\right)^{\theta} .
$$


In particular, for $p_{0}=p_{1}=p$ we have $\left(\left[X_{0}, X_{1}\right]_{[\theta]}\right)_{[p]} \equiv\left[X_{0[p]}, X_{1[p]}\right]_{[\theta]}$.

Let us recall that if $Z$ is an order continuous Banach function space, then the bounded measurable functions are dense in $Z$. If $f \in Z$ and $f_{n}:=f \chi_{A_{n}}$ with $A_{n}:=\{\omega \in \Omega:|f(\omega)| \leq n\}$ then $\left(f_{n}\right)_{n}$ converges to $f$ in $Z_{[p]}$ for every $1 \leq p<\infty$.

Theorem 5.3. Let $\left(X_{0}, X_{1}\right)$ be a pair of order continuous Banach function spaces over the finite measure $\mu$, and let $\left(E_{0}, E_{1}\right)$ be a pair of Banach spaces. Let $T:\left(X_{0}, X_{1}\right) \rightarrow\left(E_{0}, E_{1}\right)$ be a $\mu$-determined operator. Then, for each $1 \leq p<\infty$ and each $0<\theta<1$

$$
\left[L^{p}\left(m_{0}\right), L^{p}\left(m_{1}\right)\right]_{[\theta]} \subseteq L^{p}\left(m_{\theta}\right),
$$

where the vector measures $m_{0}, m_{1}$ and $m_{\theta}$ are respectively the ones associated with $T_{0}, T_{1}$ and $T_{\theta}:\left[X_{0}, X_{1}\right]_{[\theta]} \rightarrow\left[E_{0}, E_{1}\right]_{[\theta]}$.

Proof. In virtue of Proposition 1.1(3), for $i=0,1$ and $0<\theta<1$, the integration maps $I_{m_{i}}: L^{1}\left(m_{i}\right) \rightarrow E_{i}$ and $I_{m_{\theta}}: L^{1}\left(m_{\theta}\right) \rightarrow E_{\theta}$ are the unique extensions of $T_{i}$ and $T_{\theta}$ respectively. Let $f \in L^{1}\left(m_{0}\right) \cap L^{1}\left(m_{1}\right)$, then there exists $k \in \mathbb{N}$ and $f_{k}:=f \chi_{A_{k}}$ where $A_{k}:=\{\omega \in \Omega$ : $|f(\omega)| \leq k\}$ such that $2\left\|I_{m_{i}}\right\|\left\|f-f_{k}\right\|_{L^{1}\left(m_{i}\right)}<\varepsilon$ for $i=0,1$. Therefore

$$
\left\|I_{m_{0}}(f)-I_{m_{1}}(f)\right\|_{E_{0}+E_{1}} \leq\left\|I_{m_{0}}\left(f-f_{k}\right)\right\|_{E_{0}}+\left\|I_{m_{1}}\left(f-f_{k}\right)\right\|_{E_{1}}<\varepsilon .
$$

Then $I_{m_{0}}$ and $I_{m_{1}}$ coincide in the intersection of their domains, and so one can define the interpolation operator. On the other hand, since $X_{i} \subseteq L^{1}\left(m_{i}\right)$, it is easy to check that $\left[X_{0}, X_{1}\right]_{[\theta]} \subseteq\left[L^{1}\left(m_{0}\right), L^{1}\left(m_{1}\right)\right]_{[\theta]}$. If we denote $I_{\theta}$ the interpolation of the integration maps $I_{m_{i}}$, we have the following diagrams
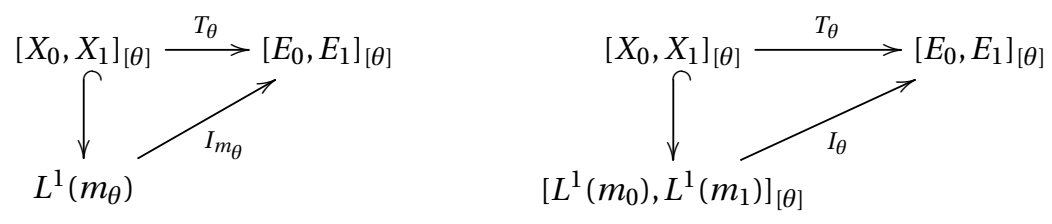

DIAGRAM 5.1. Applying of the optimal domain theorem to the interpolation operator

Now, by the optimal domain Theorem 1.2 we have that $\left[L^{1}\left(m_{0}\right), L^{1}\left(m_{1}\right)\right]_{[\theta]} \subseteq L^{1}\left(m_{\theta}\right)$. So by this and by Remark 5.2 we obtain

$$
\begin{aligned}
{\left[L^{p}\left(m_{0}\right), L^{p}\left(m_{1}\right)\right]_{[\theta]} } & =\left[L^{1}\left(m_{0}\right)_{[1 / p]}, L^{1}\left(m_{1}\right)_{[1 / p]}\right]_{[\theta]} \\
& =\left(\left[L^{1}\left(m_{0}\right), L^{1}\left(m_{1}\right)\right]_{[\theta]}\right)_{[1 / p]} \subseteq L^{1}\left(m_{\theta}\right)_{[1 / p]}=L^{p}\left(m_{\theta}\right) .
\end{aligned}
$$

And the proof is completed.

Without the assumption of $\mu$-determination, but with other requirements, we can obtain a $p$-th power factorization for the interpolated operator. 
Proposition 5.4. Let $T:\left(X_{0}, X_{1}\right) \rightarrow\left(E_{0}, E_{1}\right)$ be an operator from a pair $\left(X_{0}, X_{1}\right)$ of order continuous Banach function spaces over a finite measure into a pair of Banach spaces $\left(E_{0}, E_{1}\right)$. If the restrictions $T_{0}$ and $T_{1}$ are $p$-th power factorable for some $1<p<\infty$, then the interpolated operator $T_{\theta}:\left[X_{0}, X_{1}\right]_{[\theta]} \rightarrow\left[E_{0}, E_{1}\right]_{[\theta]}$ is $p$-th power factorable for every $0<\theta<1$.

Proof. Step 1. Since the restrictions $T_{0}: X_{0} \rightarrow E_{0}$ and $T_{1}: X_{1} \rightarrow E_{1}$ can be extended to $X_{0[p]}$ and $X_{1[p]}$ respectively, we are going to prove that these extensions allow us to define an operator $X_{0[p]}+X_{1[p]} \rightarrow E_{0}+E_{1}$ such that its restrictions to $X_{0[p]}$ and $X_{1[p]}$ are the operators that extend $T_{0}$ and $T_{1}$ respectively.

By definition of $p$-th power factorable operator, for $i=0,1, T_{i}=T_{i[p]} \circ j_{[p]}$, where $j_{[p]}$ denotes the inclusion $X_{i} \subseteq X_{i[p]}$ and $T_{i[p]}: X_{i[p]} \rightarrow E_{0}$ is the extension map. It is straightforward to prove that $T_{0[p]}$ and $T_{1[p]}$ coincide on $X_{0[p]} \cap X_{1[p]}$. Denote by $M_{i}, i=0,1$ the quasi-norm of $T_{i[p]}: X_{i[p]} \rightarrow E_{i}$. Let us take $f \in X_{0[p]} \cap X_{1[p]}$ and $\varepsilon>0$. Since $X_{0[p]}$ and $X_{1[p]}$ are order continuous we can obtain $k \in \mathbb{N}$ such that

$$
\left\|f-f_{k}\right\|_{X_{0[p]}}<\frac{\varepsilon}{2 M_{0}} \quad \text { and }\left\|f-f_{k}\right\|_{X_{1[p]}}<\frac{\varepsilon}{2 M_{1}}
$$

where $f_{k}:=f \chi_{A_{k}}$ with $A_{k}:=\{\omega \in \Omega:|f(\omega)| \leq k\}$. Hence, having in mind that $T_{i[p]}\left(f_{k}\right)=$ $T_{i}\left(f_{k}\right)=T\left(f_{k}\right), i=0,1$, we have

$$
\left\|T_{0[p]}(f)-T_{1[p]}(f)\right\|_{E_{0}+E_{1}} \leq\left\|T_{0[p]}(f)-T\left(f_{k}\right)\right\|_{E_{0}}+\left\|T\left(f_{k}\right)-T_{1[p]}(f)\right\|_{E_{1}}<\varepsilon .
$$

Thus we have that $T_{0[p]}(f)=T_{1[p]}(f)$ and so $\left.T_{0[p]}\right|_{X_{0[p]} \cap X_{1[p]}}=\left.T_{1[p]}\right|_{X_{0[p]} \cap X_{1[p]}}$.

Step 2. Let us now define the map

$$
\begin{array}{rll}
\widetilde{T}: \quad X_{0[p]}+X_{1[p]} & \longrightarrow E_{0}+E_{1} \\
f_{0}+f_{1} & \longrightarrow T_{0[p]}\left(f_{0}\right)+T_{1[p]}\left(f_{1}\right) .
\end{array}
$$

Let us see that it is well defined. Assume we have two representations of an element in $X_{0[p]}+X_{1[p]}$, i.e. $g_{0}+g_{1}=f_{0}+f_{1}$ where $f_{i}, g_{i} \in X_{i[p]}$ for $i=0,1$. Thus, $T_{0[p]}\left(g_{0}-f_{0}\right)=$ $T_{1[p]}\left(f_{1}-g_{1}\right)$ since $g_{0}-f_{0}=g_{1}-f_{1} \in X_{0[p]} \cap X_{1[p]}$, hence by linearity of $T_{i[p]}, \widetilde{T}\left(g_{0}+g_{1}\right)=$ $\widetilde{T}\left(f_{0}+f_{1}\right)$. Moreover if $f_{0}+f_{1} \in X_{0}+X_{1}$, we have that $\widetilde{T}\left(f_{0}+f_{1}\right)=T_{0}\left(f_{0}\right)+T_{1}\left(f_{1}\right)=T\left(f_{0}\right)+$ $T\left(f_{1}\right)=T\left(f_{0}+f_{1}\right)$. It is easy to check that this map is linear and continuous, since $T_{i[p]}$ is.

From these arguments, $\left.\widetilde{T}\right|_{X_{i[p]}}=T_{i[p]}$ for $i=0,1$, so we obtain the interpolation map

$$
(\widetilde{T})_{\theta}:\left(\left[X_{0}, X_{1}\right]_{[\theta]}\right)_{[p]} \rightarrow\left[E_{0}, E_{1}\right]_{[\theta]},
$$

since $\left[X_{0[p]}, X_{1[p]}\right]_{[\theta]}=\left(\left[X_{0}, X_{1}\right]_{[\theta]}\right)_{[p]}$ (see Remark 5.2). Moreover $\left.(\widetilde{T})_{\theta}\right|_{\left[X_{0}, X_{1}\right]_{[\theta]}}=T_{\theta}$, since $(\widetilde{T})_{\theta}$ and $T_{\theta}$ are restrictions of $\widetilde{T}$ and $T$ respectively, and $\left[X_{0}, X_{1}\right]_{[\theta]} \subseteq X_{0}+X_{1}$. Therefore, we have an extension of $T_{\theta}$ to its $p$-th power space, which is the definition of $p$-th power factorable operator (see comments below from the Definition 1.6). 
For the case in what $T_{0}$ and $T_{1}$ are respectively, $p_{0}$-th and $p_{1}$-th power factorable for $p_{0} \neq p_{1}$, we can assume an inclusion between the codomains $E_{0}$ and $E_{1}$ in order to obtain a factorization for the interpolated operator.

Proposition 5.5. Let $T:\left(X_{0}, X_{1}\right) \rightarrow\left(E_{0}, E_{1}\right)$ be an operator from a pair $\left(X_{0}, X_{1}\right)$ of order continuous Banach function spaces over a finite measure into a pair of Banach spaces $\left(E_{0}, E_{1}\right)$. If the restrictions $T_{0}$ and $T_{1}$ are respectively $p_{0}$-th and $p_{1}$-th power factorable with $1 \leq p_{0} \leq$ $p_{1}<\infty$, and $E_{0} \subseteq E_{1}$, then the interpolated operator $T_{\theta}:\left[X_{0}, X_{1}\right]_{[\theta]} \rightarrow\left[E_{0}, E_{1}\right]_{[\theta]}$ is $p_{\theta}$-th power factorable for every $0<\theta<1$.

Proof. Since $p_{0} \leq p_{1}$, we have $\alpha:=\theta p_{0} / \widetilde{p_{\theta}} \leq \theta$. Hence $E_{0} \subseteq\left[E_{0}, E_{1}\right]_{[\alpha]} \subseteq\left[E_{0}, E_{1}\right]_{[\theta]} \subseteq$ $E_{1}$, since $E_{0} \subseteq E_{1}$ (see [9, Th. 4.2.1]).

Step 1. Since the restrictions $T_{0}: X_{0} \rightarrow E_{0}$ and $T_{1}: X_{1} \rightarrow E_{1}$ can be extended to $X_{0\left[p_{0}\right]}$ and $X_{1\left[p_{1}\right]}$ respectively, we are going to prove that these extensions allow us to define an operator $X_{0\left[p_{0}\right]}+X_{1\left[p_{1}\right]} \rightarrow E_{0}+E_{1}$ such that its restrictions to $X_{0\left[p_{0}\right]}$ and $X_{1[p]}$ are the operators that extend $T_{0}$ and $T_{1}$ respectively. This step is like the Step 1 of the previous proposition, but in this case we obtain that $\left.T_{0\left[p_{0}\right]}\right|_{X_{0\left[p_{0}\right]} \cap X_{1\left[p_{1}\right]}}=\left.T_{1\left[p_{1}\right]}\right|_{X_{0\left[p_{0}\right]} \cap X_{1\left[p_{1}\right]}}$.

Step 2. Let us now define the following map

$$
\begin{array}{rll}
\widetilde{T}: \quad X_{0\left[p_{0}\right]}+X_{1\left[p_{1}\right]} & \longrightarrow & E_{0}+E_{1} \\
f_{0}+f_{1} & \longrightarrow T_{0\left[p_{0}\right]}\left(f_{0}\right)+T_{1\left[p_{1}\right]}\left(f_{1}\right) .
\end{array}
$$

It is simple to show that this map is linear, continuous and well defined. On one hand, from these arguments $\left.\widetilde{T}\right|_{X_{i\left[p_{i}\right]}}=T_{i\left[p_{i}\right]}$ for $i=0,1$, so for every $0<\delta<1$ (in particular for $\delta=\theta p_{0} / \widetilde{p_{\theta}}$ ) we obtain the interpolated map

$$
\widetilde{T}_{\delta}:\left[X_{0\left[p_{0}\right]}, X_{1\left[p_{1}\right]}\right]_{[\delta]} \longrightarrow\left[E_{0}, E_{1}\right]_{[\delta]} .
$$

On the other hand, in virtue of Lemma 5.1(2) and order continuity of $X_{0}$ and $X_{1}$ we have

$$
\left(\left[X_{0}, X_{1}\right]_{[\theta]}\right)_{\left[p_{\theta}\right]}=\left[X_{0\left[p_{0}\right]}, X_{1\left[p_{1}\right]}\right]_{[\alpha]},
$$

where $\alpha=\theta p_{0} / \widetilde{p_{\theta}}$. Now, since $\alpha \leq \theta$ and $E_{0} \subseteq E_{1}$ we have that $\left[E_{0}, E_{1}\right]_{[\alpha]} \subseteq\left[E_{0}, E_{1}\right]_{[\theta]}$, thus the following diagram holds

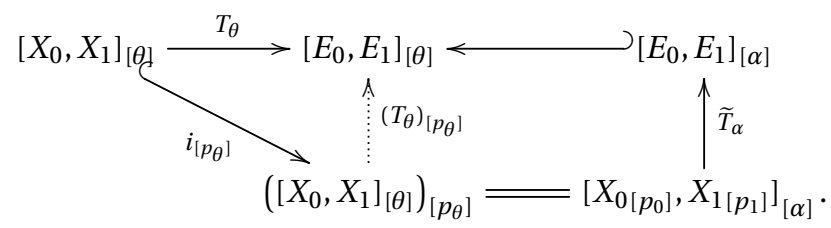

DIAGRAM 5.2. $p_{\theta}$-th power factorization for the interpolated operator $T_{\theta}$

By definition of $\widetilde{T}, T_{\theta}=\widetilde{T} \circ i_{\left[p_{\theta}\right]}=\widetilde{T}_{\alpha} \circ i_{\left[p_{\theta}\right]}$, by uniqueness of the extension $\left(T_{\theta}\right)_{\left[p_{\theta}\right]}$ and we have that $T_{\theta}$ is $p_{\theta}$-th power factorable. 
Remark 5.6. Observe that if in the proposition above we assume $p_{1} \leq p_{0}$ and $E_{1} \subseteq E_{0}$, then we obtain the same conclusion. Thus if $E_{0}=E_{1}$ one may consider every pair of indexes $1 \leq p_{0}, p_{1}<\infty$. If $p_{0}=p_{1}$, then $\alpha:=\theta p_{0} / \widetilde{p_{\theta}}=\theta$ and therefore $\left[E_{0}, E_{1}\right]_{[\theta]}=\left[E_{0}, E_{1}\right]_{[\alpha]}$, as in Theorem 5.3.

5.1.3. Interpolation for $(p, q)$-th power factorable operators. Let us now study the case of a $(p, q)$-th power factorable operator. First, when the indexes of factorization of the operators coincide, and then when they differ. In the first case we does not need any special hypothesis, however if the indexes of factorization are different in the two operators, we need to assume some restriction on the indexes.

Theorem 5.7. Let $T:\left(X_{0}, X_{1}\right) \rightarrow\left(Y_{0}, Y_{1}\right)$ be an operator between two pairs $\left(X_{0}, X_{1}\right)$ and $\left(Y_{0}, Y_{1}\right)$ of order continuous Banach function spaces over a finite measure. Assume that $Y_{0}$ and $Y_{1}$ are Fatou and that $Y_{0}^{\prime}$ and $Y_{1}^{\prime}$ are order continuous. If the restrictions $T_{0}$ and $T_{1}$ are $(p, q)$-th power factorable and $\left(Y_{0}^{\prime}\right)^{q}$ and $\left(Y_{1}^{\prime}\right)^{q}$ are order continuous for some $1 \leq p, q<\infty$, then the interpolated operator $T_{\theta}:\left[X_{0}, X_{1}\right]_{[\theta]} \rightarrow\left[Y_{0}, Y_{1}\right]_{[\theta]}$ is $(p, q)$-th power factorable for every $0<\theta<1$.

Proof. Let us first note that for each $i=0,1$, since $T_{i}$ is $(p, q)$-th power factorable, we have that $T_{i}$ is $p$-th power factorable and $T^{\prime}$ is $q$-th power factorable (Remark 4.9). Thus $\left(X_{i}\right)_{[p]}$ and $\left(Y_{i}^{\prime}\right)_{[q]}$ are nontrivial for $i=0,1$ (see Remark 4.3).

From Theorem 4.4, for $i=0,1$ there exist operators $T_{i[p, q]}: X_{i[p]} \rightarrow\left(\left(Y_{i}^{\prime}\right)_{[q]}\right)^{\prime}$ that extend $T_{i}$. By applying the argument used in Step 1 of the previous theorems we obtain that

$$
\left.T_{0[p, q]}\right|_{X_{0[p]} \cap X_{1[p]}}=\left.T_{1[p, q]}\right|_{X_{0[p]} \cap X_{1[p]}} .
$$

Now the operator $\widetilde{T}: X_{0[p]}+X_{1[p]} \rightarrow\left(\left(Y_{0}^{\prime}\right)_{[q]}\right)^{\prime}+\left(\left(Y_{1}^{\prime}\right)_{[q]}\right)^{\prime}$ defined by $\widetilde{T}\left(f_{0}+f_{1}\right):=T_{0[p, q]}\left(f_{0}\right)+$ $T_{1[p, q]}\left(f_{1}\right)$, is well defined. From these reasons we can write that $\left.\widetilde{T}\right|_{X_{i[p]}}=T_{i[p, q]}$ for $i=0,1$ and obtain the interpolated map $\widetilde{T}_{\theta}:\left[X_{0[p]}, X_{1[p]}\right]_{[\theta]} \rightarrow\left[\left(\left(Y_{0}^{\prime}\right)_{[q]}\right)^{\prime},\left(\left(Y_{1}^{\prime}\right)_{[q]}\right)^{\prime}\right]_{[\theta]}$.

We now are going to relate the domain and codomain of $\widetilde{T}_{\theta}$ with $\left(\left[X_{0}, X_{1}\right]_{[\theta]}\right)_{[p]}$ and $\left(\left(\left[Y_{0}, Y_{1}\right]_{[\theta]}^{\prime}\right)_{[q]}\right)^{\prime}$ respectively. Since both $X_{0}$ and $X_{1}$ are order continuous, so are $X_{0[p]}$ and $X_{1[p]}$, by Remark 5.2 we have that $\left(\left[X_{0}, X_{1}\right]_{[\theta]}\right)_{[p]}=\left[X_{0[p]}, X_{1[p]}\right]_{[\theta]}$.

We claim and show that

$$
\left[\left(\left(Y_{0}^{\prime}\right)_{[q]}\right)^{\prime},\left(\left(Y_{1}^{\prime}\right)_{[q]}\right)^{\prime}\right]_{[\theta]} \subseteq\left[\left(Y_{0}^{\prime}\right)_{[q]},\left(Y_{1}^{\prime}\right)_{[q]}\right]_{[\theta]}^{\prime} .
$$

Observe that by hypothesis, for $i=0,1$, we have $\left(Y_{i}^{\prime}\right)^{q}$ is order continuous, thus $\left(\left(Y_{i}^{\prime}\right)^{q}\right)_{[q]}=$ $\left(\left(Y_{i}^{\prime}\right)_{[q]}\right)^{\prime}$ is also order continuous (recall Proposition 1.5(5) and (11)). Thus the bounded functions are dense in both $\left(\left(Y_{0}^{\prime}\right)_{[q]}\right)^{\prime}$ and $\left(\left(Y_{1}^{\prime}\right)_{[q]}\right)^{\prime}$, consequently so is the intersection of these spaces. Now we can apply [9, Th. 4.3.1 and Th. 4.5.1] in order to satisfy the second 
inclusion of the following two inclusions

$$
\left[\left(Y_{0}^{\prime}\right)_{[q]},\left(Y_{1}^{\prime}\right)_{[q]}\right]_{[\theta]} \subseteq\left[\left(\left(Y_{0}^{\prime}\right)_{[q]}\right)^{\prime \prime},\left(\left(Y_{1}^{\prime}\right)_{[q]}\right)^{\prime \prime}\right]_{[\theta]} \subseteq\left[\left(\left(Y_{0}^{\prime}\right)_{[q]}\right)^{\prime},\left(\left(Y_{1}^{\prime}\right)_{[q]}\right)^{\prime}\right]_{[\theta]}^{\prime} .
$$

Applying Proposition 2.4(3) for $p=1$, we obtain (5.3).

Thanks to Lozanowskiĭ duality and order continuity of $Y_{i}$ and $Y_{i}^{\prime}$, we deduce that

$$
\left[Y_{0}, Y_{1}\right]_{[\theta]}^{\prime}=\left(Y_{0}^{1-\theta} Y_{1}^{\theta}\right)^{\prime}=\left(Y_{0}^{\prime}\right)^{1-\theta}\left(Y_{1}^{\prime}\right)^{\theta}=\left[Y_{0}^{\prime}, Y_{1}^{\prime}\right]_{[\theta]} .
$$

Thus, from order continuity of $Y_{0}^{\prime}$ and $Y_{1}^{\prime}$, applying again Lemma 5.1(1) we have that

$$
\left(\left[Y_{0}, Y_{1}\right]_{[\theta]}^{\prime}\right)_{[q]}=\left(\left[Y_{0}^{\prime}, Y_{1}^{\prime}\right]_{[\theta]}\right)_{[q]}=\left[\left(Y_{0}^{\prime}\right)_{[q]},\left(Y_{1}^{\prime}\right)_{[q]}\right]_{[\theta]} .
$$

Finally, since $Y_{0}$ and $Y_{1}$ have the Fatou property, taking duals in the last equality and by (5.3) we obtain that

$$
\begin{aligned}
{\left[\left(\left(Y_{0}^{\prime}\right)_{[q]}\right)^{\prime},\left(\left(Y_{1}^{\prime}\right)_{[q]}\right)^{\prime}\right]_{[\theta]} } & \subseteq\left[\left(Y_{0}^{\prime}\right)_{[q]},\left(Y_{1}^{\prime}\right)_{[q]}\right]_{[\theta]}^{\prime}=\left(\left(\left[Y_{0}, Y_{1}\right]_{[\theta]}^{\prime}\right)_{[q]}\right)^{\prime} \\
& \subseteq\left[Y_{0}, Y_{1}\right]_{[\theta]}^{\prime \prime}=\left(Y_{0}^{1-\theta} Y_{1}^{\theta}\right)^{\prime \prime}=\left(Y_{0}^{\prime \prime}\right)^{1-\theta}\left(Y_{1}^{\prime \prime}\right)^{\theta}=Y_{0}^{1-\theta} Y_{1}^{\theta}=\left[Y_{0}, Y_{1}\right]_{[\theta]} .
\end{aligned}
$$

Diagram 5.3 below clarifies the situation.

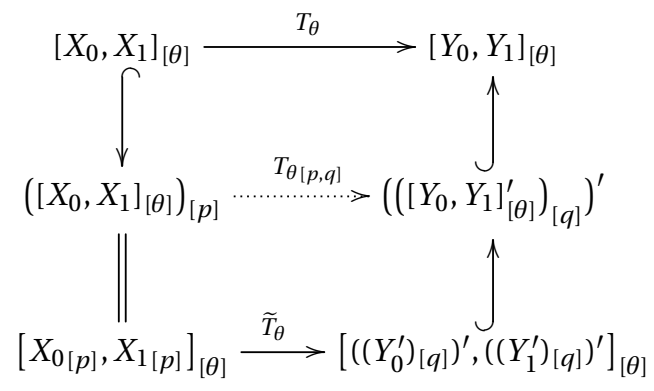

DIAGRAM 5.3. Interpolation for extensions of $(p, q)$-th power factorable operators

In consequence it is obtained the operator

$$
T_{\theta[p, q]}:\left(\left[X_{0}, X_{1}\right]_{[\theta]}\right)_{[p]} \rightarrow\left(\left(\left[Y_{0}, Y_{1}\right]_{[\theta]}^{\prime}\right)_{[q]}\right)^{\prime} .
$$

Moreover, from the order continuity and Fatou hypothesis for the Banach function spaces involved and by the Lozanowskiu duality we have that $\left[X_{0}, X_{1}\right]_{[\theta]}$ and $\left[Y_{0}, Y_{1}\right]_{[\theta]}^{\prime}$ are order continuous and that $\left[Y_{0}, Y_{1}\right]_{[\theta]}$ (is order continuous and) has the Fatou property. This implies, thanks to Theorem 4.4 , that $T_{\theta}$ is $(p, q)$-th power factorable.

The last theorem uses the same technique to interpolate the extension maps and shows the most general result that we reach in this section. 
Theorem 5.8. Let $T:\left(X_{0}, X_{1}\right) \rightarrow\left(Y_{0}, Y_{1}\right)$ be an operator, such that $\left(X_{0}, X_{1}\right)$ and $\left(Y_{0}, Y_{1}\right)$ be two pairs of order continuous Banach function spaces over a finite measure. Assume that $Y_{0}$ and $Y_{1}$ are Fatou and $Y_{0}^{\prime}$ and $Y_{1}^{\prime}$ are order continuous. If the restrictions $T_{0}$ and $T_{1}$ are respectively $\left(p_{0}, q_{0}\right)$-th and $\left(p_{1}, q_{1}\right)$-th power factorable and $\left(Y_{0}^{\prime}\right)^{q_{0}}$ and $\left(Y_{1}^{\prime}\right)^{q_{1}}$ are order continuous for $1 \leq p_{0}, p_{1}, q_{0}, q_{1}<\infty$ with $\operatorname{det}\left(\begin{array}{c}p_{0} \\ q_{0} \\ q_{1}\end{array}\right)=0$, then the interpolated operator $T_{\theta}$ is $\left(p_{\theta}, q_{\theta}\right)$-th power factorable for every $0<\theta<1$.

Proof. As in the previous theorem $X_{i}$ and $Y_{i}^{\prime}$ are order continuous and we deduce that $X_{i\left[p_{i}\right]}$ and $Y_{i\left[q_{i}\right]}^{\prime}$ are non-trivial for $i=0,1$, since $T_{i}: X_{i} \rightarrow Y_{i}$ and $T_{i}^{\prime}: Y_{i}^{\prime} \rightarrow X_{i}^{\prime}$ are $p_{i}$ th and $q_{i}$-th power factorable for $i=0,1$. By hypothesis $T_{0}$ is $\left(p_{0}, q_{0}\right)$-th power factorable, so by Theorem 4.4 there exits $T_{0\left[p_{0}, q_{0}\right]}: X_{0\left[p_{0}\right]} \rightarrow\left(\left(Y_{0}^{\prime}\right)_{\left[q_{0}\right]}\right)^{\prime}$, and the same for $T_{1}$, there exists $T_{1\left[p_{1}, q_{1}\right]}: X_{1\left[p_{1}\right]} \rightarrow\left(\left(Y_{1}^{\prime}\right)_{\left[q_{1}\right]}\right)^{\prime}$.

We now are going to prove that the map

$$
\widetilde{T}:\left(X_{0\left[p_{0}\right]}, X_{1\left[p_{1}\right]}\right) \rightarrow\left(\left(\left(Y_{0}^{\prime}\right)_{\left[q_{0}\right]}\right)^{\prime},\left(\left(Y_{1}^{\prime}\right)_{\left[q_{1}\right]}\right)^{\prime}\right),
$$

defined by $\widetilde{T}\left(f_{0}+f_{1}\right):=T_{0\left[p_{0}, q_{0}\right]}\left(f_{0}\right)+T_{1\left[p_{1}, q_{1}\right]}\left(f_{1}\right)$, is well defined. To this we only need to prove that $T_{0\left[p_{0}, q_{0}\right]}(f)=T_{1\left[p_{1}, q_{1}\right]}(f)$ for every $f \in X_{0\left[p_{0}\right]} \cap X_{1\left[p_{1}\right]}$.

Let $f \in X_{0\left[p_{0}\right]} \cap X_{1\left[p_{1}\right]}$. As in the previous theorem $X_{0} \cap X_{1}$ is dense in $X_{0\left[p_{0}\right]} \cap X_{1\left[p_{1}\right]}$, then there exists a sequence $\left(f_{n}\right)_{n} \subset X_{0} \cap X_{1}$ such that $f_{n} \rightarrow f$ in $X_{0\left[p_{0}\right]} \cap X_{1\left[p_{1}\right]}$. By continuity we have that $T_{i\left[p_{i}, q_{i}\right]}\left(f_{n}\right) \rightarrow T_{i\left[p_{i}, q_{i}\right]}(f)$ for $i=0,1$. On the other hand, again as in the previous theorems, we know that $\left.T_{0\left[p_{0}, q_{0}\right]}\right|_{X_{0} \cap X_{1}}=\left.T_{1\left[p_{1}, q_{1}\right]}\right|_{X_{0} \cap X_{1}}$, and so

$$
0=\left(T_{0\left[p_{0}, q_{0}\right]}-T_{1\left[p_{1}, q_{1}\right]}\right)\left(f_{n}\right) \rightarrow\left(T_{0\left[p_{0}, q_{0}\right]}-T_{1\left[p_{1}, q_{1}\right]}\right)(f)=0 .
$$

Therefore $\left.T_{0\left[p_{0}, q_{0}\right]}\right|_{X_{0\left[p_{0}\right]} \cap X_{1\left[p_{1}\right]}}=\left.T_{1\left[p_{1}, q_{1}\right]}\right|_{X_{0\left[p_{0}\right]} \cap X_{1\left[p_{1}\right]}}$ Then $\widetilde{T}$ is well defined (linear and continuous), and so we may consider the interpolation operator $\widetilde{T}_{\alpha}$ for $\alpha \in(0,1)$. The next step is to identify the domain and codomain of $\widetilde{T}_{\alpha}$ with the domain and codomain respectively of the hypothetic extension $\left(T_{\theta}\right)_{\left[p_{\theta}, q_{\theta}\right]}:\left(\left[X_{0}, X_{1}\right]_{[\theta]}\right)_{\left[p_{\theta}\right]} \rightarrow\left(\left(\left[Y_{0}, Y_{1}\right]_{[\theta]}^{\prime}\right)_{\left[q_{\theta}\right]}\right)^{\prime}$.

In virtue of Lemma 5.1(2) and order continuity of $X_{0}$ and $X_{1}$ we obtain that

$$
\left(\left[X_{0}, X_{1}\right]_{[\theta]}\right)_{\left[p_{\theta}\right]}=\left[X_{0\left[p_{0}\right]}, X_{1\left[p_{1}\right]}\right]_{[\alpha]},
$$

for $\alpha:=\theta p_{0} / \widetilde{p_{\theta}}$. Again as in the theorem above we apply Lozanowskii duality and order continuity of $Y_{i}$ and $Y_{i}^{\prime}$ to obtain that $\left[Y_{0}, Y_{1}\right]_{[\theta]}^{\prime}=\left[Y_{0}^{\prime}, Y_{1}^{\prime}\right]_{[\theta]}$. We now apply Lemma 5.1(2), which gives that $\left(\left[Y_{0}, Y_{1}\right]_{[\theta]}^{\prime}\right)_{\left[q_{\theta}\right]}=\left[\left(Y_{0}^{\prime}\right)_{\left[q_{0}\right]},\left(Y_{1}^{\prime}\right)_{\left[q_{1}\right]}\right]_{[\beta]}$, where $\beta:=\theta q_{0} / \widetilde{q_{\theta}}$. By taking duals and having in mind that $\left(Y_{0}^{\prime}\right)^{q_{0}}$ and $\left(Y_{1}^{\prime}\right)^{q_{1}}$ are order continuous, we use the same arguments that prove (5.3) of the previous theorem with [9, Th. 4.5.1 and Th. 4.3.1], and so we have

$$
\left[\left(\left(Y_{0}^{\prime}\right)_{\left[q_{0}\right]}\right)^{\prime},\left(\left(Y_{1}^{\prime}\right)_{\left[q_{1}\right]}\right)^{\prime}\right]_{[\beta]} \subseteq\left(\left[\left(Y_{0}^{\prime}\right)_{\left[q_{0}\right]},\left(Y_{1}^{\prime}\right)_{\left[q_{1}\right]}\right]_{[\beta]}\right)^{\prime}=\left(\left(\left[Y_{0}, Y_{1}\right]_{[\theta]}^{\prime}\right)_{\left[q_{\theta}\right]}\right)^{\prime} .
$$

Observe that since $Y_{0}$ and $Y_{1}$ are Fatou, $\left[Y_{0}, Y_{1}\right]_{[\theta]}$ is Fatou. 
Let us show that $\alpha=\beta$. For $0<\theta<1$, developing $\widetilde{q_{\theta}}$ in this equality, we obtain $\frac{p_{0}}{\widetilde{p_{\theta}}}=\frac{q_{0}}{\widetilde{q_{\theta}}}$ if and only if

$$
\frac{p_{1} \theta}{p_{0}(1-\theta)+p_{1} \theta}=\frac{q_{1} \theta}{q_{0}(1-\theta)+q_{1} \theta},
$$

i.e. $\theta(1-\theta)\left(p_{1} q_{0}-p_{0} q_{1}\right)=0$, that is $\operatorname{det}\left(\begin{array}{ll}p_{0} & p_{1} \\ q_{0} & q_{1}\end{array}\right)=0$, which is our hypothesis.

Summarizing, if we define $T_{\theta\left[p_{\theta}, q_{\theta}\right]}:=i \circ \widetilde{T}_{\alpha}$ we have the following extension map

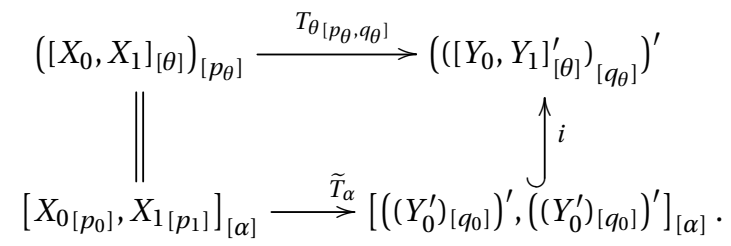

Finally, in virtue of Theorem 4.4, the interpolated operator $T_{\theta}:\left[X_{0}, X_{1}\right]_{[\theta]} \rightarrow\left[Y_{0}, Y_{1}\right]_{[\theta]}$ is $\left(p_{\theta}, q_{\theta}\right)$-th power factorable.

Remark 5.9. Finally we note that the technique of interpolate the extensions maps can be applied to other factorizations as for example Maurey-Rosenthal, one only needs to verify if the interpolation of convex/concave spaces is convex/concave in the same sense. Also we can try to interpolate using the real method using known results for the K-functional of $p$-convexifications (see e.g. [70]). The second complex method does not seem to give us more information since for the interpolation of $p$-integrable function spaces with respect to a vector measure, both methods coincide to each other (see [40]).

\section{2. $(p, q)$-th power factorable kernel operators}

The statements provided in this section give tools for the study of the optimal domain and optimal range of a kernel operator, that are sometimes called Hille-Tamarkin operators. Recall that a kernel operator $T_{K}: X \rightarrow Y$ between Banach function spaces is a Hille-Tamarkin operator if \|\|$K\left\|_{X^{\prime}, \omega_{2}}\right\|_{Y}<\infty$ (see Section 1.2.2).

An important fact is that most of the usual kernel operators are defined in sets of infinite measure and so the main requirement regarding the measure in this work is not fulfilled. The results that have been obtained are for finite measure spaces. However notice that, as was explained at Section 4.2, Lemma 4.13 and Corollary 4.14 allow to adapt them to the case of $\sigma$-finite measures, obtaining in this way canonical factorizations for operators as the one that is shown in the last example of this section. For other studies on kernel operators from the point of view of the optimal domain and factorization we cite $[29,17,28]$.

Hereafter, $(\Omega, \Sigma, \mu)$ will be a finite measure space. Clearly, the map $A \rightsquigarrow \int_{A} K d \mu \otimes d \mu$, $A \in \Sigma \otimes \Sigma$ defines a measure denoted by $\eta$ (see Example 4.12). So we have the product measure space $(\Omega \times \Omega, \Sigma \otimes \Sigma, \eta)$. We will use the notation described in Section 1.2.2. 
5.2.1. Hölder's type inequality. Under the requirements of Theorem 4.7, assuming some power factorability of the adjoint and the operator itself, the next results show that a Hölder's type inequality implies $(p, q)$-th power factorability. We follow the notation of Example 4.12, namely if $f, g$ are real valued functions defined on $\Omega$, then $(f \otimes g)\left(\omega_{1}, \omega_{2}\right):=$ $f\left(\omega_{1}\right) g\left(\omega_{2}\right)$.

Theorem 5.10. Let $X$ and $Y$ be Banach function spaces. Assume that $X$ and $Y^{\prime}$ are order continuous and $Y$ is Fatou. Let $K \in L^{1}(\mu \otimes \mu)$ such that its associated kernel map $T_{K}: X \rightarrow Y$ is continuous. Suppose that for some $1<p, q<\infty$, the following conditions are satisfied:

(i) $T_{K}$ is $p^{2}$-th power factorable.

(ii) $T_{K}^{\prime}$ is $q^{2}$-th power factorable.

(iii) There exists $C>0$ such that for every $f \in X$ and $g \in Y^{\prime}$,

$$
\|g \otimes f\|_{L^{1}(\eta)} \leq C\left\|\chi_{\Omega} \otimes f\right\|_{L^{p}(\eta)}\left\|g \otimes \chi_{\Omega}\right\|_{L^{q}(\eta)},
$$

where $d \eta:=K d \mu \otimes d \mu$.

Then $T_{K}$ is $(p, q)$-th power factorable.

Proof. For the aim of clarity we write $d x$ and $d y$ instead of $d \mu\left(\omega_{1}\right)$ and $d \mu\left(\omega_{2}\right)$. In consequence, by hypothesis and Fubini's Theorem, for $f \in X$ and $g \in Y^{\prime}$ we have that

$$
\begin{aligned}
\left|\left\langle T_{K}(f), g\right\rangle\right| & =\left|\int_{\Omega}\left(\int_{\Omega} f(y) K(x, y) d y\right) g(x) d x\right|=\left|\int_{\Omega \times \Omega} f(y) g(x) d \eta\right| \\
& \leq\|g \otimes f\|_{L^{1}(\eta)} \leq C\left\|\chi_{\Omega} \otimes f\right\|_{L^{p}(\eta)}\left\|g \otimes \chi_{\Omega}\right\|_{L^{q}(\eta)} .
\end{aligned}
$$

Let us now estimate the last two factors. Again by Fubini's Theorem and the fact that $T_{K}$ is $p^{2}$-th power factorable, we have

$$
\begin{aligned}
\left\|\chi_{\Omega} \otimes f\right\|_{L^{p}(\eta)}^{p}= & \int_{\Omega \times \Omega}\left|\chi_{\Omega} \otimes f\right|^{p} K d x \otimes d y=\int_{\Omega}\left(\int_{\Omega}|f(y)|^{p} K(x, y) d y\right) \chi_{\Omega}(x) d x \\
& \leq\left\|\chi_{\Omega}\right\|_{Y^{\prime}} \sup _{u \in B_{Y^{\prime}}} \int_{\Omega}\left(\int_{\Omega}|f(y)|^{p} K(x, y) d y\right) u(x) d x=\left\|\chi_{\Omega}\right\|_{Y^{\prime}} \sup _{u \in B_{Y^{\prime}}}\left|\left\langle T_{K}\left(|f|^{p}\right), u\right\rangle\right| \\
& =\left\|\chi_{\Omega}\right\|_{Y^{\prime}}\left\|T_{K}\left(|f|^{p}\right)\right\|_{Y} \leq\left\|\chi_{\Omega}\right\|_{Y^{\prime}} Q_{1}\left\||f|^{1 / p}\right\|_{X}^{p^{2}}=\left\|\chi_{\Omega}\right\|_{Y^{\prime}} Q_{1}\|f\|_{X_{[p]}}^{p} .
\end{aligned}
$$

Analogously, since $T_{K}^{\prime}$ is $q^{2}$-th power factorable, we obtain

$$
\left\|g \otimes \chi_{\Omega}\right\|_{L^{q}(\eta)}^{q}=\int_{\Omega \times \Omega}\left|g \otimes \chi_{\Omega}\right|^{q} d \eta \leq\left\|\chi_{\Omega}\right\|_{X} Q_{2}\left\||g|^{1 / q}\right\|_{Y^{\prime}}^{q^{2}}=\left\|\chi_{\Omega}\right\|_{X} Q_{2}\|g\|_{\left(Y^{\prime}\right)_{[q]}}^{q} .
$$

Finally Theorem 4.7 implies that $T_{k}$ is a $(p, q)$-th power factorable operator.

Hölder-Rogers inequality and Theorem 1.9 provide us the following corollary.

Corollary 5.11. Let $X$ and $Y$ be Banach function spaces over the finite measure $\mu$. Let $K \in L^{1}(\mu \otimes \mu)$ be a positive kernel such that the associated kernel operator $T_{K}: X \rightarrow Y$ is $\mu$-determined. Assume that $X$ and $Y^{\prime}$ are order continuous and $Y$ is Fatou. Suppose that $1<p, q<\infty$ are such that the following conditions are satisfied: 
(i) $\frac{1}{p}+\frac{1}{q} \leq 1$.

(ii) $T_{K}$ is $p^{2}$-th power factorable.

(iii) $T_{K}^{\prime}$ is $q^{2}$-th power factorable.

Then $T_{K}$ is $(p, q)$-th power factorable. Moreover, if $\frac{1}{p}+\frac{1}{q}=1$, then $T_{K}$ factors through $L^{p}$. spaces of scalar measures, i.e. the following diagram commutes

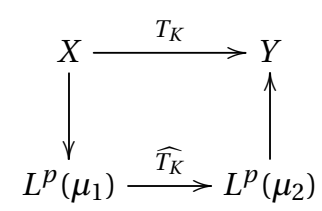

DiAGRAM 5.4. Factorization through $L^{p}$-spaces for kernel operators

where $\mu_{1}$ and $\mu_{2}$ are positive finite scalar measures.

Proof. The first statement is simple since $\frac{1}{p}+\frac{1}{q} \leq 1$, hence taking into account that $g \otimes f=\left(g \otimes \chi_{\Omega}\right)\left(\chi_{\Omega} \otimes f\right)$, Hölder-Rogers inequality yields immediately the condition (5.5) of Theorem 5.10, which gives the result.

For the second statement, by Theorem 5.10 and Theorem 4.7(5) we obtain $H_{K}$, an extension of $T_{K}$ from $L^{p}\left(m_{R}\right)$ into $\left(L^{q}\left(m_{S^{\prime}}\right)\right)^{\prime}$, where $R$ and $S$ are operators such that $T_{K}=$ $S \circ R$. In fact, the proof of Theorem 5.10 makes clear that $R$ and $S^{\prime}$ can be chosen to be positive. On the other hand, since $T_{K}$ is a positive operator, so is $H_{K}$, and we obtain by [66, Prop. 1.d.9], for every set of functions $f_{1}, \ldots, f_{n} \in L^{p}\left(m_{R}\right)$,

$$
\left\|\left(\sum_{i=1}^{n}\left|H_{K}\left(f_{i}\right)\right|^{p}\right)^{1 / p}\right\|_{\left(L^{q}\left(m_{S^{\prime}}\right)\right)^{\prime}} \leq\left\|H_{K}\right\|\left\|\left(\sum_{i=1}^{n}\left|f_{i}\right|^{p}\right)^{1 / p}\right\|_{L^{p}\left(m_{R}\right)} .
$$

This implies by Theorem 1.9 the factorization diagram for two measures $\mu_{1}$ and $\mu_{2}$ that are Rybakov measures for $m_{R}$ and $m_{S^{\prime}}$, respectively.

Remark 5.12. Let $1 \leq p<\infty$. Let $K: \Omega \times \Omega \rightarrow[0, \infty)$ be a kernel function. Let $T_{K}: X \rightarrow Y$ the kernel operator associated with $K$, where $X$ and $Y$ are Banach function spaces. Let $d \eta=K d \mu \otimes d \mu$ be the measure defined above. For a given $f \in X$,

$\int_{\Omega} T_{K}\left(|f|^{p}\right)\left(\omega_{1}\right) d \mu(x)=\int_{\Omega} \chi_{\Omega}\left(\omega_{1}\right) \int_{\Omega}\left|f\left(\omega_{2}\right)\right|^{p} K\left(\omega_{1}, \omega_{2}\right) d \mu\left(\omega_{2}\right) d \mu\left(\omega_{1}\right)=\int_{\Omega \times \Omega}\left|\chi_{\Omega} \otimes f\right|^{p} d \eta$.

Therefore $\chi_{\Omega} \otimes X:=\left\{\chi_{\Omega} \otimes f: f \in X\right\} \subseteq L^{p}(\eta)$ if and only if $T_{K}\left(X_{[p]}\right) \subseteq L^{1}(\mu)$, understanding this last expression as the kernel operator applied to functions of the $p$-th power space. It happens for example when $T_{K}$ is $p$-th power factorable, since $T_{K}\left(X_{[p]}\right) \subseteq Y \subseteq L^{1}(\mu)$. The same argument for $Y^{\prime}$ brings us to that $Y^{\prime} \otimes \chi_{\Omega}:=\left\{g \otimes \chi_{\Omega}: g \in Y^{\prime}\right\} \subseteq L^{q}(\eta)$ if and only if $T_{K}^{\prime}\left(\left(Y^{\prime}\right)_{[q]} \subseteq L^{1}(\mu)\right.$, with the same abuse of notation. 
5.2.2. Hille-Tamarkin operators. Let us now present another condition concerning the boundedness for the kernel function which implies with other natural assumptions the $(p, q)$-th power factorability of the corresponding kernel operator. The following results can be applied for instance in the case of Hille-Tamarkin operators.

We start with a proposition that gives sufficient conditions for the factorization of kernel operators through the adequate power space. Recall that all along this section every Banach function space is based over a finite measure.

Proposition 5.13. Let $(\Omega, \Sigma, \mu)$ be a finite measure space and let $1 \leq r, s, p, q<\infty$ be such that $\frac{1}{r}=\frac{1}{p}+\frac{1}{s}$ and let $K$ be a kernel function such that \|\|$K\left\|_{q, \omega_{1}}\right\|_{s / r}<\infty$, i.e.

$$
\left(\int_{\Omega}\left(\int_{\Omega}\left|K\left(\omega_{1}, \omega_{2}\right)\right|^{q} d \mu\left(\omega_{2}\right)\right)^{s / q r} d \mu\left(\omega_{1}\right)\right)^{r / s}<\infty .
$$

Then the kernel operator associated with $K, T_{K}: L^{p}(\mu) \rightarrow L^{q}(\mu)$ is $r$-th power factorable.

Proof. Since $r<p, L^{p} \subseteq L^{r}$. Let $f$ be a simple function. We will use $d x$ and $d y$ instead of $d \mu\left(\omega_{1}\right)$ and $d \mu\left(\omega_{2}\right)$. Then, using Minkowski's integral inequality (1.8) and HölderRogers inequality we obtain

$$
\begin{aligned}
\left(\left\|T_{K} f\right\|_{q}\right)^{1 / r} & =\left(\int_{\Omega}\left|\int_{\Omega} K(x, y) f(y) d y\right|^{q} d x\right)^{1 /(q r) \stackrel{(1.8)}{\leq}}\left(\int_{\Omega}\left(\int_{\Omega}|K(x, y) f(y)|^{q} d x\right)^{1 / q} d y\right)^{1 / r} \\
& =\left(\int_{\Omega}|f(y)|\left(\int_{\Omega}|K(x, y)|^{q} d x\right)^{1 / q} d y\right)^{1 / r}=\left(\int_{\Omega}|f(y)|\|K\|_{q, \omega_{1}}(y) d y\right)^{1 / r} \\
& \leq\left(\int_{\Omega}|f(y)|^{p / r} d y\right)^{1 / p}\left(\int_{\Omega}\|K\|_{q, \omega_{1}}^{s / r}(y) d y\right)^{1 / s}=\left\|\left(\|K\|_{q, \omega_{1}}\right)^{1 / r}\right\|\left\|_{s}\right\||f|^{1 / r} \|_{p} .
\end{aligned}
$$

Using that simple functions are dense in $L^{p}$, these inequalities prove that there is a constant $C:=\left\|\left(\|K\|_{q, \omega_{1}}\right)^{1 / r}\right\|_{s}$ such that $\left\|T_{K} g\right\|_{q} \leq C\left\||g|^{1 / r}\right\|_{p}^{r}=C\|g\|_{L^{p}(\mu)_{[r]}}$ for all $g \in L^{p}(\mu)$. Thus we have that $T_{K}: L^{p}(\mu) \rightarrow L^{q}(\mu)$ is $r$-th power factorable.

Example 5.14 (The Volterra operator). The Volterra operator $V_{p}: L^{p}[0,1] \rightarrow L^{p}[0,1](p>$ 1) has kernel function $K(x, y):=\chi_{[0, x]}(y)$, hence for $r, s>1$ such that $\frac{1}{r}=\frac{1}{p}+\frac{1}{s}$ we have

$$
\begin{aligned}
\|\| K\left\|_{q, \omega_{1}}\right\|_{s / r} & =\left(\int_{0}^{1}\left(\int_{0}^{1}\left|\chi_{[0, x]}(y)\right|^{q} d x\right)^{s / q r} d y\right)^{r / s}=\left(\int_{0}^{1}\left(\int_{0}^{1}\left|\chi_{[y, 1]}(x)\right|^{q} d x\right)^{s / q r} d y\right)^{r / s} \\
& =\left(\int_{0}^{1}\left(\int_{y}^{1} d x\right)^{s / q r} d y\right)^{r / s}=\left(\int_{0}^{1}(1-y)^{s / q r} d y\right)^{r / s} \\
& \left.=\left(\int_{0}^{1} t^{s / q r} d t\right)^{r / s}=\left(\frac{t^{(s / q r)+1}}{\frac{s}{q r}+1}\right]_{0}^{1}\right)^{r / s}=\left(\frac{q r}{q r+s}\right)^{r / s} .
\end{aligned}
$$

Then by Proposition 5.13, $V_{p}$ is $r$-th power factorable for $r \in$ [1, $p$ ], see [79, Ex. 5.9].

Proposition 5.15. Let $X$ and $Y$ be Banach function spaces over the Lebesgue measure on $[0,1]$ such that $X$ and $Y^{\prime}$ are order continuous and $Y$ is Fatou. Suppose that $X \subseteq L^{p^{2}}[0,1]$ and $Y^{\prime} \subseteq L^{q^{2}}[0,1]$. Let $1<p, q<\infty$ and let $T_{K}: X \rightarrow Y$ be a kernel operator with kernel 
function $K:[0,1] \times[0,1] \rightarrow[0, \infty)$ such that \|\|$K\left\|_{p^{\prime}, \omega_{2}}\right\|_{q^{\prime}}<\infty$. Then $T_{K}$ is $(p, q)$-th power factorable.

Proof. By hypothesis, $X_{[p]} \subseteq L^{p}[0,1]$ and $\left(Y^{\prime}\right)_{[q]} \subseteq L^{q}[0,1]$. Let $f \in X$ and $g \in Y^{\prime}$. Applying the Hölder-Rogers inequality twice, we obtain

$$
\begin{aligned}
& \left|\left\langle T_{K} f, g\right\rangle\right|=\left|\int_{0}^{1} \int_{0}^{1} K(x, y) f(y) g(x) d y d x\right| \leq \int_{0}^{1}|g(x)| \int_{0}^{1}|K(x, y) f(y)| d y d x \\
& \quad \leq \int_{0}^{1}|g(x)|\|K\|_{p^{\prime}, \omega_{2}}(x)\|f\|_{p} d x \leq\|\| K\left\|_{p^{\prime}, \omega_{2}}\right\|_{q^{\prime}}\|g\|_{q}\|f\|_{p} \leq C\|\| K\left\|_{p^{\prime}, \omega_{2}}\right\|_{q^{\prime}}\|f\|_{X_{[p]}}\|g\|_{Y^{\prime}[q]} .
\end{aligned}
$$

Now, since \|\|$K\left\|_{p^{\prime}, \omega_{2}}\right\|_{q^{\prime}}<\infty$ we can apply Theorem 4.7 in order to finish the proof.

Example 5.16 (Hartley kernel). Let us show that the Hartley transform, when applied to certain Orlicz spaces over finite measure, is always $(p, q)$-th power factorable for every $p, q>1$. This transform was proposed in 1942 [51] as an alternative to the Fourier transform with the advantage that it maps real valued functions into real valued functions. It has applications to some current transmission problems and it has the form

$$
\int_{-N}^{N}(\cos (x y)+\sin (x y)) f(y) d y, \quad f \in L^{1}[-N, N] .
$$

Since we are working in the context of finite measure and positive kernel operators we will use the so called absolute kernel $K(x, y):=|\cos (x y)+\sin (x y)|$, for $x, y \in[-N, N]$, which is always positive (see [108, Ch. $13 \$ 94]$ for the relation between kernel operators and absolute kernel operators). Since $|\cos (t)+\sin (t)| \leq \sqrt{2}$, we have that $\|K\|_{p^{\prime}, \omega_{1}} \leq \sqrt{2}(2 N)^{1 / p^{\prime}}$ for every $1<p<\infty$, this implies that

$$
\|\| K\left\|_{p^{\prime}, \omega_{1}}\right\|_{q^{\prime}} \leq \sqrt{2}\left\|(2 N)^{1 / p^{\prime}}\right\|_{q^{\prime}}=\sqrt{2}(2 N)^{1 / p^{\prime}+1 / q^{\prime}}<\infty,
$$

for every $1<q<\infty$. Let us consider an Orlicz space $L^{\varphi}[-N, N]$ such that $L^{\varphi}[-N, N] \subseteq$ $L^{p^{2}}[-N, N]$ (it suffices that $|u|^{p^{2}} \leq C \varphi(u)$ ). We also consider an Orlicz space $L^{\psi}[-N, N]$ such that $\left(L^{\psi}[-N, N]\right)^{\prime} \subseteq L^{q^{2}}[-N, N]$ (in this case it suffices that $|u|^{q^{2}} \leq D$ sup $\{2 u \nu-\psi(\nu)$ : $v>0\}$, see $\left[12\right.$, Rem. 6.8]). Suppose that $T_{K}: L^{\varphi}[-N, N] \rightarrow L^{\psi}[-N, N]$, the operator associated with $K$, is continuous (see [60, Th. III.15.1 and Lem. III.15.1] for the existence of the Orlicz functions $\varphi$ and $\psi$ ). Finally by applying Proposition 5.15 for the interval $[-N, N]$ instead of $[0,1]$, we obtain that the absolute Hartley kernel operator is $(p, q)$-th power factorable for every $1<p, q<\infty$.

The following proposition is a version of Proposition 5.13 for $(p, q)$-th power factorable operators. It represents the canonical example of this class of operators.

Proposition 5.17. Let $(\Omega, \Sigma, \mu)$ be a finite measure space and let $1<s \leq 2 \leq r<\infty$. Let $T_{K}: L^{r}(\mu) \rightarrow L^{s}(\mu)$ be a kernel operator with kernel function such that \|\|$K\left\|_{s^{\prime}, \omega_{1}}\right\|_{r}<\infty$, i.e.

$$
\left(\int_{\Omega}\left(\int_{\Omega}\left|K\left(\omega_{1}, \omega_{2}\right)\right|^{s^{\prime}} d \mu\left(\omega_{1}\right)\right)^{r / s^{\prime}} d \mu\left(\omega_{2}\right)\right)^{1 / r}<\infty .
$$


Then there is $C>0$ such that

$$
\left|\left\langle T_{K} f, g\right\rangle\right| \leq C\|f\|_{r^{\prime}}\|g\|_{s}, \quad f \in L^{r}(\mu), g \in L^{s^{\prime}}(\mu) .
$$

Thus, $T_{K}$ is $\left(r-1, s^{\prime}-1\right)$-th power factorable.

Proof. Let $f \in L^{r}$ and $g \in L^{s^{\prime}}$. As usual we write $d x$ and $d y$ instead of $d \mu\left(\omega_{1}\right)$ and $d \mu\left(\omega_{2}\right)$. Applying Fubini's Theorem and Hölder-Rogers inequality twice, we have

$$
\begin{aligned}
\left|\left\langle T_{K} f, g\right\rangle\right| & \leq \int_{\Omega}|f(y)|\left(\int_{\Omega}|K(x, y) g(x)| d x\right) d y=\int_{\Omega}|f(y)|\|K \cdot g\|_{1, \omega_{1}}(y) d y \\
& \leq\|g\|_{s} \int_{\Omega}|f(y)|\|K\|_{s^{\prime}, \omega_{1}}(y) d y \leq\|g\|_{s}\|f\|_{r^{\prime}}\|\| K\left\|_{s^{\prime}, \omega_{1}}\right\|_{r} .
\end{aligned}
$$

Since $\frac{r}{r^{\prime}} \geq 1$ and $\frac{s^{\prime}}{s} \geq 1$ we can write $L^{r^{\prime}}=\left(L^{r}\right)_{\left[r / r^{\prime}\right]}$ and $L^{s}=\left(L^{s^{\prime}}\right)_{\left[s^{\prime} / s\right]}$, therefore Theorem 4.7 implies that $T_{K}$ is $\left(r / r^{\prime}, s^{\prime} / s\right)$-th power factorable.

The last corollary in this chapter allows us to obtain a $(p, q)$-th power factorable kernel operator whose adjoint is a Hille-Tamarkin operator by means of the dual relation between their kernels.

Corollary 5.18. Let $1<s \leq 2 \leq r<\infty$. Let $K:[0,1] \times[0,1] \rightarrow[0, \infty)$ be a kernel function, and let $K^{\prime}(x, y):=K(y, x)$ be its dual kernel. Let $T_{K}: L^{r}[0,1] \rightarrow L^{s}[0,1]$ be the kernel operator associated with $K$. If the kernel operator $T_{K^{\prime}}: L^{s}[0,1] \rightarrow L^{r}[0,1]$ associated with $K^{\prime}$ is HilleTamarkin, then $T_{K}$ is $\left(r-1, s^{\prime}-1\right)$-th power factorable. If in addition $r^{\prime} \leq s$, then $T_{K}$ is Hille-Tamarkin.

Proof. $T_{K^{\prime}}: L^{s}[0,1] \rightarrow L^{r}[0,1]$ is Hille-Tamarkin, thus \|\|$K\left\|_{s^{\prime}, \omega_{1}}\right\|_{r}=\|\| K^{\prime}\left\|_{s^{\prime}, \omega_{2}}\right\|_{r}<$ $\infty$. By Proposition 5.17, $T_{K}$ is $\left(r-1, s^{\prime}-1\right)$-th power factorable. For the second statement we use the Minkowski's integral inequality in his form with norms (1.9), which we will be able to apply since $r^{\prime} \leq s$. Observe also that $r^{\prime} \leq r$ and $s \leq s^{\prime}$, thus $L^{r}[0,1] \subseteq L^{r^{\prime}}[0,1]$ and $L^{s^{\prime}}[0,1] \subseteq L^{s}[0,1]$. Therefore we have

$$
\|\| K\left\|_{r^{\prime}, \omega_{2}}\right\|_{s} \leq\|\| K^{\prime}\left\|_{r^{\prime}, \omega_{1}}\right\|_{s} \stackrel{(1.9)}{\leq}\|\| K^{\prime}\left\|_{s, \omega_{2}}\right\|_{r^{\prime}} \leq Q_{1}\|\| K^{\prime}\left\|_{s, \omega_{2}}\right\|_{r} \leq Q_{1} Q_{2}\|\| K^{\prime}\left\|_{s^{\prime}, \omega_{2}}\right\|_{r}<\infty,
$$

where $Q_{1}, Q_{2}>0$. This implies that $T_{K}: L^{r}[0,1] \rightarrow L^{s}[0,1]$ is a Hille-Tamarkin operator. 


\section{CHAPTER 6}

\section{AN OPTIMAL DOMAIN FOR THE LAPLACE TRANSFORM}

We finish the work with a construction of the optimal domain for the Laplace transform assuming that it takes values on a particular weighted $L^{p}$-space based on a finite measure. In general, given a kernel operator that takes values on a $\sigma$-finite based $L^{p}$ space, namely $L^{p}[0, \infty)$, we define an isometric map towards $L^{p}(h d x)$, a finite based space by a weight function $h$, so that $m_{p, h}$, the $L^{p}(h d x)$-valued vector measure associated with the kernel operator is well defined. By means of this vector measure we have that the kernel operator can be extended to the canonical optimal domain $L^{1}\left(m_{p, h}\right)$, in fact we obtain a bound of the operator norm for the restrictions to $L^{q}\left(m_{p, h}\right)$ that can be taken as small as we want depending on the selected weight from a concrete family of weights. Finally we apply Theorem 6.9 to modulate this optimal domain and weighted codomain in order to provide a repertory of spaces where the kernel operator is well defined.

\subsection{Introduction}

In order to obtain the optimal domain as has been described above we will study when the Laplace transform is $(p, q)$-th power factorable when considered between two given Banach function spaces that we will construct. Concretely, we will compute the double norm of the Laplace kernel in such spaces in order to apply the preceding results. We will show that it is sufficient this double norm be finite in order to have a factorization in our sense. An important fact is that in general the usual kernel operators are defined in sets of infinite measure (see e.g. $[\mathbf{1 0}, \mathbf{4 7}, \mathbf{4 8}]$ for the Laplace transform case). This is the reason for that, in order to apply our results, we will make use of a weight function that brings to a finite measure the infinite Lebesgue measure. The Laplace transform is often defined to be of complex variable, but here we will study the real variable case. It is defined as the following improper integral

$$
\mathscr{L} f(x):=\int_{0}^{\infty} f(y) e^{-x y} d y, \quad x \in[0, \infty) .
$$

The convergence of this transform has been widely studied ([74], [109], [104] among a lot of others). The associated kernel function is $K_{\mathscr{L}}(x, y):=e^{-x y}$ for $(x, y) \in[0, \infty) \times[0, \infty)$. We will show that the kernel of the Laplace operator satisfies a condition for being $(p, q)$-th power factorable in the adequate context. If the operator defined by a kernel satisfies some conditions, the theory of vector measures provide us the so called optimal domain - the 
space of integrable functions $-L^{1}(m)$ where $m$ is a concrete vector measure depending on the kernel operator (see [79, Ch. 4] and references therein). In order to be precise, $m(A):=$ $\mathscr{L}\left(\chi_{A}\right)$ for all $A \in \Sigma$. All along this chapter we are working with three integral norms, in order to distinguish them we use $\|\cdot\|_{L^{p}(\mu)},\|\cdot\|_{L^{p}(m)}$ and $\|\cdot\|_{p}$ for the norms corresponding to a finite measure $\mu$, a vector measure $m$ and the Lebesgue measure, respectively ( $p \in$ $[1, \infty])$.

Regarding the Laplace transform, as far as we know no systematic study of the optimal range/domain has been done, although there are several recent results in this direction where they approach the problem from several points of view but for other kernel operators (see e.g. $[\mathbf{1 7}, \mathbf{1 9}, \mathbf{2 8}, \mathbf{2 9}, \mathbf{3 0}, \mathbf{7 5}, \mathbf{8 0}, \mathbf{8 1}, \mathbf{7 9}]$ ). In this work we center our attention in the case when the range space is a weighted $L^{p}$ space, that appears in a natural way (see [10]), but now using vector measure techniques for describing the optimal domain as an $L^{1}(m)$ for a particular vector measure $m$ (see [31] for a description of this space). We prove several results on the structure of such space, mainly the continuous inclusion of $L^{q}[0, \infty)$ spaces in it.

In Theorem 6.7 we fix a concrete space of integrable functions as the set where the functions that are representable as Laplace transforms may belong. In the next step we analyze conditions to obtain the vector measure associated with the Laplace transform taking values in that space, in order to use the description of its optimal domain. This provides a bigger order continuous space where the Laplace transform works. Finally, in Example 6.15, we factorize the Laplace kernel operator so we find spaces of integrable functions with respect to scalar measures where the Laplace transform is continuous.

Let us remark that the classical study of the Laplace transform is oriented to find conditions for representing a function as a Laplace integral or to analyze the inverse problem. In [105, Ch. VII] it was shown that the Laplace transform maps $L^{p}[0, \infty)$ (for $p \in[1, \infty]$ ) into a subset in $C^{\infty}(0, \infty)$, (see e.g. [5] for more information about this representation of real and vector-valued functions). These results are based in the Post's conditions $[\mathbf{8 9}, \mathrm{Th}$. XXI] for a Laplace representation of real valued functions. Other points of view can be found in [72] and [7].

\subsection{The vector measure}

Let us consider the $\sigma$-finite measure space of the set $[0, \infty)$ with the Lebesgue measure and the $\sigma$-algebra of Lebesgue measurable sets of [0, $\infty)$. Let us denote by $K_{\mathscr{L}}(x, y):=e^{-x y}$ the Laplace kernel and let $1 \leq p<\infty$ be a fixed value. Recall that $\int_{0}^{\infty} e^{-a x} d x=\frac{1}{a}$ for $a>0$. Then we choose $h \in L^{1}[0, \infty) \cap L^{1}\left(\frac{d x}{x^{p}}\right)$, and so we have the following condition

$$
\begin{aligned}
\|\| K_{\mathscr{L}}\left\|_{1, \omega_{2}}\right\|_{L^{p}(h d x)} & =\left(\int_{0}^{\infty}\left(\int_{0}^{\infty} e^{-x y} d y\right)^{p} h(x) d x\right)^{1 / p} \\
& =\left(\int_{0}^{\infty} \frac{h(x)}{x^{p}} d x\right)^{1 / p}=\|h\|_{L^{1}\left(\frac{d x}{x^{p}}\right)}<\infty .
\end{aligned}
$$


Observe that this is the Hille-Tamarkin condition for kernel operators (see Section 1.2.2). Then we can rewrite this condition saying that the Laplace transform is continuous from $L^{\infty}[0, \infty)$ into $L^{p}(h d x)$. Taking this domain and range as starting point, the next step is to obtain some other domains and ranges. Therefore, if $0<h \in L^{1}[0, \infty) \cap L^{1}\left(\frac{d x}{x^{p}}\right)$, we define the scalar positive, finite measure $\mu_{h}: \Sigma \rightarrow[0, \infty)$ by $\mu_{h}(A):=\int_{A} h d x$ and so $d \mu_{h}$ denotes $h d x$.

For a given $1 \leq p<\infty$ and $h \in L^{1}[0, \infty) \cap L^{1}\left(\frac{d x}{x^{p}}\right)$, let us consider $p \leq r<\infty$. Since $p \leq r$ by the Minkowski's integral inequality (1.8) we have

$$
\|\| K_{\mathscr{L}}\left\|_{L^{p}\left(\mu_{h}\right), \omega_{1}}\right\|_{r}<\infty .
$$

It is provided by a simple application of the Minkowski's integral inequality and (6.1). Taking into account that $\frac{p}{r} \leq p$, we obtain

$$
\begin{aligned}
\|\| K_{\mathscr{L}}\left\|_{L^{p}\left(\mu_{h}\right), \omega_{1}}\right\|_{r} & =\left(\int_{0}^{\infty}\left(\int_{0}^{\infty}\left(e^{-x y}\right)^{p} h(x) d x\right)^{r / p} d y\right)^{1 / r} \\
& \leq\left(\int_{0}^{\infty}\left(\int_{0}^{\infty}\left(e^{-x y}\right)^{r} d y\right)^{p / r} h(x) d x\right)^{1 / p} \\
& \leq\left(\int_{0}^{\infty}\left(\frac{1}{r x}\right)^{p / r} h(x) d x\right)^{1 / p}=\frac{1}{r^{1 / r}}\left\|\frac{1}{x}\right\|_{L^{p / r}\left(\mu_{h}\right)}^{1 / r} \leq C\left\|\frac{1}{x}\right\|_{L^{p}\left(\mu_{h}\right)}^{1 / r}<\infty,
\end{aligned}
$$

where $C>0$ depends on $r$ and $p$. Then we obtain the measure $A \in \Sigma \rightsquigarrow \int_{A}\left\|K_{\mathscr{L}}\right\|_{L^{p}\left(\mu_{h}\right), \omega_{1}}^{r} d t$ (see e.g. [45, Sect. 31]). For the aim of simplicity we have assumed that $h$ is continuous, but it can be chosen piecewise continuous almost everywhere.

Example 6.1. For $q \in[p, \infty)$, the function $h(x):=x^{q} e^{-x}>0$ in $[0, \infty)$, is continuous and belongs to $L^{1}[0, \infty) \cap L^{1}\left(\frac{d x}{x^{p}}\right)$.

Theorem 6.2. Let $1 \leq p<\infty$. Let $0<h \in L^{1}[0, \infty) \cap L^{1}\left(\frac{d x}{x^{p}}\right)$ be a continuous function (which depends on $p$ ) and let $\mu_{h}$ be the measure defined above. Then the set function $m_{p, h}: \Sigma \rightarrow$ $L^{p}\left(\mu_{h}\right)$, defined by $m_{p, h}(A):=\mathscr{L}\left(\chi_{A}\right)$, is a vector measure.

Proof. Let us first verify that $m_{p, h}(A) \in L^{p}\left(\mu_{h}\right)$, for all $A \in \Sigma$. Applying (6.1) we have that $m_{p, h}$ is well defined, since

$$
\begin{aligned}
\left\|m_{p, h}(A)\right\|_{L^{p}\left(\mu_{h}\right)}^{p} & =\int_{0}^{\infty}\left|m_{p, h}(A)(x)\right|^{p} h(x) d x=\int_{0}^{\infty}\left(\int_{A} e^{-x y} d y\right)^{p} h(x) d x \\
& \leq \int_{0}^{\infty}\left(\int_{0}^{\infty} e^{-x y} d y\right)^{p} h(x) d x=\int_{0}^{\infty} \frac{h(x)}{x^{p}} d x<\infty .
\end{aligned}
$$

We continue the proof by showing that $m_{p, h}$ is $\sigma$-additive. Obviously $m_{p, h}$ es finitely additive. Let $\left(A_{k}\right)_{k} \subseteq \Sigma$ be a pairwise disjoint sequence. We define $B_{n}:=\cup_{k=1}^{n} A_{k}$ for each $0<n \in \mathbb{N}$ and $A:=\cup_{k=1}^{\infty} A_{k}$. Let us prove that for each $x>0$ it is truth that

$$
\mathscr{L}\left(\chi_{B_{n}}\right)(x) \rightarrow \mathscr{L}\left(\chi_{A}\right)(x) .
$$

Clearly, for each $y>0$

$$
e^{-x y} \chi_{B_{n}}(y) \rightarrow e^{-x y} \chi_{A}(y) .
$$


Since for every $x, y>0$ we have that $e^{-x y} \chi_{B_{n}}(y) \leq e^{-x y} \chi_{A}(y)$ then, taking into account that the function $e^{-x y} \chi_{A}(y) \in L^{1}[0, \infty)$ for each $x>0$, by the Lebesgue's Dominated Convergence Theorem we obtain

$$
\mathscr{L}\left(\chi_{B_{n}}\right)(x)=\int_{0}^{\infty} e^{-x y} \chi_{B_{n}}(y) d y \rightarrow \mathscr{L}\left(\chi_{A}\right)(x)=\int_{0}^{\infty} e^{-x y} \chi_{A}(y) d y .
$$

That is $\mathscr{L}\left(\chi_{B_{n}}\right)$ converge to $\mathscr{L}\left(\chi_{A}\right)$ pointwise. Thus, it converges in measure for the finite measure $\mu_{h}$, by the Egoroff's Theorem. Since for each $0<n \in \mathbb{N}$ we have that $0 \leq \mathscr{L}\left(\chi_{B_{n}}\right) \leq$ $\mathscr{L}\left(\chi_{A}\right) \in L^{p}\left(\mu_{h}\right)$, the sequence $\left(\mathscr{L}\left(\chi_{B_{n}}\right)\right)_{n}$ is uniformly integrable with respect to $\mu_{h}$. If we apply the Vitali's Convergence Theorem ([33, Th. III.15]) we have that $\mathscr{L}\left(\chi_{B_{n}}\right)$ converges to $\mathscr{L}\left(\chi_{A}\right)$ in $L^{p}\left(\mu_{h}\right)$, or in other words $m_{p, h}$ is countably additive.

For the following corollary see [79, Th. 4.14].

Corollary 6.3. Let $1 \leq p<\infty$. Under the same hypothesis of Theorem 6.2 , the integration map associated with $m_{p, h}$ is in fact the Laplace transform defined on its optimal domain, $I_{m_{p, h}}=\mathscr{L}: L^{1}\left(m_{p, h}\right) \rightarrow L^{p}\left(\mu_{h}\right)$, with respect to every order continuous Banach function space $X$ over a Rybakov measure for $m_{p, h}$ such that $\mathscr{L}: X \rightarrow L^{p}\left(\mu_{h}\right)$.

6.2.1. An embedding property. Now let us see some properties of this vector measure. Notice that by definition, the measure associated with the Laplace transform depends on the parameter $p \geq 1$ and the function $h$.

Proposition 6.4. Let $1 \leq p<\infty$ and let a continuous function $h \in L^{1}[0, \infty) \cap L^{1}\left(\frac{d x}{x^{p}}\right)$. Let $m_{p, h}: \Sigma \rightarrow L^{p}\left(\mu_{h}\right)$ be the vector measure defined in Theorem 6.2. The following statements hold:

(1) The measure $m_{p, h}$ is equivalent to $\mu_{h}$ and also equivalent to the Lebesgue measure in $\Sigma$, i.e. they have the same null sets.

(2) $L^{q}[0, \infty) \subseteq L^{1}\left(m_{p, h}\right)$ for every $1 \leq q \leq \infty$.

(3) $L^{q}[0, \infty) \subseteq L^{r}\left(m_{p, h}\right)$ for every $r \leq q \leq \infty$.

Proof. (1). It is clear that $\mu_{h}$ has the same null sets as the Lebesgue measure. Let $A \in \Sigma$ be such that has Lebesgue measure zero, then $\int_{0}^{\infty} \chi_{B} d x=0$ for all $B \subseteq A$, then we fix $B$, hence

$$
m_{p, h}(B)=\mathscr{L}\left(\chi_{B}\right)=\int_{0}^{\infty} \chi_{B}(y) e^{-x y} d y=0
$$

for all $x \in[0, \infty)$ and all $B \subseteq A$, since the Laplace kernel is strictly positive. Then thanks to [79, Rem. 3.4(i)] we can conclude that $A$ is $m_{p, h}$-null. 
(2). We consider $1<q \leq \infty$. Let $f \in L^{q}(0, \infty)$ and let $0<g \in L^{p^{\prime}}\left(\mu_{h}\right)$. Applying HölderRogers inequality we have that

$$
\begin{aligned}
\int_{0}^{\infty} \mathscr{L}(|f|) g d \mu_{h} & =\int_{0}^{\infty} \int_{0}^{\infty}|f(y)| e^{-x y} d y g(x) d \mu_{h}(x) \leq\|f\|_{q}\|\| K_{\mathscr{L}}\left\|_{q^{\prime}, \omega_{2}} g\right\|_{L^{1}\left(\mu_{h}\right)} \\
& =\|f\|_{q}\left(\frac{1}{q^{\prime}}\right)^{1 / q^{\prime}}\left\|x^{-1 / q^{\prime}} g(x)\right\|_{L^{1}\left(\mu_{h}\right)} \leq\|f\|_{q}\left(\frac{1}{q^{\prime}}\right)^{1 / q^{\prime}}\left\|x^{-1 / q^{\prime}}\right\|_{L^{p}\left(\mu_{h}\right)}\|g\|_{L^{p^{\prime}}\left(\mu_{h}\right)}
\end{aligned}
$$

By definition of $\mu_{h}$, we have that $x^{-1} \in L^{p}\left(\mu_{h}\right)$, thus $x^{-1 / q^{\prime}} \in L^{p q^{\prime}}\left(\mu_{h}\right) \subseteq L^{p}\left(\mu_{h}\right)$. If $q=1$, then $q^{\prime}=\infty$ and $\left(\left\|K_{\mathscr{L}}\right\|_{q^{\prime}, \omega_{2}}\right)(x)=\sup _{y>0} e^{-x y}=1$ for every $x>0$. Hence, the bound is

$$
\begin{aligned}
\int_{0}^{\infty} \mathscr{L}(|f|) g d \mu_{h} & =\int_{0}^{\infty} \int_{0}^{\infty}|f(y)| e^{-x y} d y g(x) d \mu_{h}(x) \\
& \leq\|f\|_{1}\|\| K \mathscr{L}\left\|_{\infty, \omega_{2}} g\right\|_{L^{1}\left(\mu_{h}\right)} \leq\|f\|_{1}\left\|g \chi_{[0, \infty)}\right\|_{L^{1}\left(\mu_{h}\right)} \\
& \leq\|f\|_{1}\left\|\chi_{[0, \infty)}\right\|_{L^{p}\left(\mu_{h}\right)}\|g\|_{L^{p^{\prime}\left(\mu_{h}\right)}} \leq\|f\|_{1}\|h\|_{1}^{1 / p}\|g\|_{L^{p^{\prime}}\left(\mu_{h}\right)} .
\end{aligned}
$$

Therefore, by the computations above, we can write the following inequality

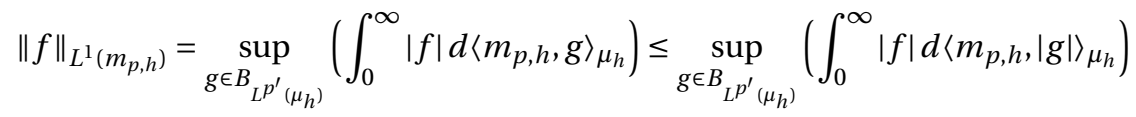

$$
\begin{aligned}
& =\sup _{g \in B_{L^{p^{\prime}}\left(\mu_{h}\right)}}\left(\int_{0}^{\infty} \mathscr{L}(|f|)|g| d \mu_{h}\right) \leq C\|f\|_{q},
\end{aligned}
$$

where $C=\left(\frac{1}{q^{\prime}}\right)^{1 / q^{\prime}}\left\|x^{-1 / q^{\prime}}\right\|_{L^{p}\left(\mu_{h}\right)}$ when $1<q \leq \infty$, and $C=\|h\|_{1}^{1 / p}$ when $q=1$. Therefore $L^{q}[0, \infty) \subseteq L^{1}\left(m_{p, h}\right)$.

(3). Let $1 \leq r \leq q$, thus $L^{q}[0, \infty)=\left(L^{q / r}[0, \infty)\right)_{[1 / r]} \subseteq\left(L^{1}\left(m_{p, h}\right)\right)_{[1 / r]}=L^{r}\left(m_{p, h}\right)$.

Remark 6.5. Clearly, for $1 \leq p<\infty$, the spaces $L^{p}\left(\mu_{h}\right)$ and $L^{p}[0, \infty)$ are isometric by means of the operators $M_{h^{1 / p}}: L^{p}\left(\mu_{h}\right) \rightarrow L^{p}[0, \infty)$ and $M_{h^{-1 / p}}: L^{p}[0, \infty) \rightarrow L^{p}\left(\mu_{h}\right)$ (recall that $h>0$ almost everywhere). Then, for $1 \leq q \leq \infty$, we have obtained the continuous and linear map $\mathscr{L}_{q, h}: L^{q}[0, \infty) \rightarrow L^{p}[0, \infty)$ defined by

$$
\left(\mathscr{L}_{p, h} g\right)(x):=h^{1 / p}(x) \int_{0}^{\infty} g(y) e^{-x y} d y
$$

where $0<h \in L^{1}[0, \infty) \cap L^{1}\left(\frac{d x}{x^{p}}\right)$ is a continuous function.

6.2.2. An estimate for the norm. Let us obtain a convenient lower estimate for the norm of the space of $p$-integrable functions with respect to the vector measure associated with a given operator.

Remark 6.6. Let $([0, \infty), \Sigma, \mu)$ be a finite measure space, $X$ is an order continuous Banach function space over $\mu$, and $E$ is a Banach function space. Let $T: X \rightarrow E$ be a positive operator, then $m_{T}: \Sigma \rightarrow E$ defined as $m_{T}(A):=T\left(\chi_{A}\right)$ is a positive vector measure (see [79, p. 184]). Let us now make a little calculation in order to obtain a convenient bound from 
below for the norm of $L^{p}\left(m_{T}\right)$, where $p \geq 1$. Let $0 \leq g \in E^{\prime}$ and let $f:=\sum_{k=1}^{n} \alpha_{k} \chi_{A_{k}}$ be a simple function. For each $k=1, \ldots, n$ we have that

$$
\begin{aligned}
\int_{0}^{\infty} \chi_{A_{k}} d\left|\left\langle m_{T}, g\right\rangle\right| & =\left|\left\langle\int_{0}^{\infty} \chi_{A_{k}} d m_{T}, g\right\rangle\right| \\
& =\left|\int_{0}^{\infty} m_{T}\left(A_{k}\right) g d \mu\right|=\left|\int_{0}^{\infty} T\left(\chi_{A_{k}}\right) g d \mu\right| .
\end{aligned}
$$

Using this, we obtain

$$
\begin{aligned}
\int_{0}^{\infty}|f|^{p} d\left|\left\langle m_{T}, g\right\rangle\right| & =\sum_{k=1}^{n}\left|\alpha_{k}\right|^{p} \int_{0}^{\infty} \chi_{A_{k}} d\left|\left\langle m_{T}, g\right\rangle\right| \\
& =\sum_{k=1}^{n}\left|\alpha_{k}\right|^{p}\left|\int_{0}^{\infty} T\left(\chi_{A_{k}}\right) g d \mu\right|=\left|\int_{0}^{\infty} T\left(|f|^{p}\right) g d \mu\right| .
\end{aligned}
$$

Let us assume that $E^{\prime}$ is a norming subspace of $E^{*}$, i.e. $\|x\|_{E}=\sup \left\{|\langle f, \xi\rangle|:\|\xi\|_{E^{\prime}} \leq 1\right\}$ for every $x \in E$. If $E$ is order continuous, then $E^{\prime}=E^{*}$ is trivially norming (see e.g. [66, Prop. 1.b.18] or [68, Th. I.I.4] for a characterization for norming Köthe dual spaces). Therefore the norm in $L^{p}\left(m_{T}\right)$ can be bounded from below in the following way

$$
\begin{aligned}
\|f\|_{L^{p}\left(m_{T}\right)} & =\sup _{g \in B_{E^{\prime}}}\left(\int_{0}^{\infty}|f|^{p} d\left|\left\langle m_{T}, g\right\rangle\right|\right)^{1 / p} \\
& \geq \sup _{0 \leq g \in B_{E^{\prime}}}\left(\int_{0}^{\infty}|f|^{p} d\left|\left\langle m_{T}, g\right\rangle\right|\right)^{1 / p} \\
& =\sup _{0 \leq g \in E^{\prime}}\left(\|g\|_{E^{\prime}}^{-1}\left|\int_{0}^{\infty} T\left(|f|^{p}\right) g d \mu\right|\right)^{1 / p} .
\end{aligned}
$$

Having in mind that the simple functions are dense in $L^{p}\left(m_{T}\right)$, we have the same formula for any $f \in L^{p}\left(m_{T}\right)$

The following outcome is an immediate consequence of Theorem 6.2. We have defined the Laplace transform as an operator from $L^{p}\left(m_{p, h}\right)$ into $L^{p}\left(\mu_{h}\right)$, where the function $0<h \in L^{1}[0, \infty) \cap L^{1}\left(\frac{d x}{x^{p}}\right)$ is continuous. In fact, this result can be extended to all positive kernel operators that satisfy condition (6.1), as can be easily seen after reading the proof. Observe that $L^{p}\left(m_{p, h}\right)=\left(L^{1}\left(m_{p, h}\right)\right)_{[1 / p]}$ is the optimal domain for the Laplace transform among the $L^{p}\left(\mu_{h}\right)$-valued extensions that are $p$-th power factorable (see [79, Th. 5.11]). Note that the following result is related to [96, Th. 3.3 and 3.4]. We will denote by $\|T\|_{X, Y}$ the norm of $T$ as operator from $X$ into $Y$.

Theorem 6.7. Let $1 \leq p<\infty$, and let a continuous function $0<h \in L^{1}[0, \infty) \cap L^{1}\left(\frac{d x}{x^{p}}\right)$. Then

$$
\|\mathscr{L}\|_{L^{p}\left(m_{p, h}\right), L^{p}\left(\mu_{h}\right)} \leq\|\| K \mathscr{L}\left\|_{1, \omega_{2}}\right\|_{L^{p}\left(\mu_{h}\right)}^{1 / p^{\prime}} .
$$

Proof. Recall that $m_{p, h}(A):=\mathscr{L}\left(\chi_{A}\right)$ and that $\mu_{h}(A):=\int_{A} h(x) d x$. Thus, we have that $\int_{0}^{\infty} g d \mu_{h}=\int_{0}^{\infty} g(x) h(x) d x$ whenever the integrals exist for $g \in L^{0}[0, \infty)$. An standard density argument leads to the solution. We define the function $0 \leq \widetilde{g}:=\left(\mathscr{L}\left(\chi_{A}\right)\right)^{p-1}=$ 
$\left(m_{p, h}(A)\right)^{p-1} \in L^{p^{\prime}}\left(\mu_{h}\right)$. Then

$$
\begin{aligned}
\|\widetilde{g}\|_{L^{p^{\prime}\left(\mu_{h}\right)}} & =\left(\int_{0}^{\infty}\left(\int_{A} e^{-x y} d y\right)^{(p-1) p^{\prime}} h(x) d x\right)^{1 / p^{\prime}} \\
& \leq\left(\int_{0}^{\infty}\left(\int_{0}^{\infty} e^{-x y} d y\right)^{p} h(x) d x\right)^{1 / p^{\prime}}=\|\| K_{\mathscr{L}}\left\|_{1, \omega_{2}}\right\|_{L^{p}\left(\mu_{h}\right)}^{p-1}<\infty,
\end{aligned}
$$

thanks to (6.2) which is a consequence of (6.1). Let $f:=\sum_{k=1}^{n} \alpha_{k} \chi_{A_{k}}$ be a simple function. Then define $A:=\cup_{k=1}^{n} A_{k}$, and let $\widetilde{g}$ be defined as above for this set $A$. So thanks to HölderRogers inequality, for every $x>0$, we obtain

$$
\begin{aligned}
|(\mathscr{L} f)(x)|^{p} & \leq\left(\sum_{k=1}^{n}\left|\alpha_{k} \int_{A_{k}} e^{-x y} d y\right|\right)^{p}=\left(\sum_{k=1}^{n}\left|\alpha_{k}\right|\left|\int_{A_{k}} e^{-x y} d y\right|^{1 / p}\left|\int_{A_{k}} e^{-x y} d y\right|^{1 / p^{\prime}}\right)^{p} \\
& \leq\left(\sum_{k=1}^{n}\left|\alpha_{k}\right|^{p}\left|\int_{A_{k}} e^{-x y} d y\right|^{p / p}\right)^{p / p}\left(\sum_{k=1}^{n}\left|\int_{A_{k}} e^{-x y} d y\right|^{p^{\prime} / p^{\prime}}\right)^{p / p^{\prime}} \\
& =\left(\sum_{k=1}^{n}\left|\alpha_{k}\right|^{p}\left|\int_{A_{k}} e^{-x y} d y\right|\right)\left(\sum_{k=1}^{n}\left|\int_{A_{k}} e^{-x y} d y\right|\right)^{p / p^{\prime}} \\
& =\left(\sum_{k=1}^{n}\left|\alpha_{k}\right|^{p}\left|\int_{A_{k}} e^{-x y} d y\right|\right)\left|\int_{A} e^{-x y} d y\right|^{p-1}=\left(\mathscr{L}\left(|f|^{p}\right)(x) \widetilde{g}(x) .\right.
\end{aligned}
$$

Finally we apply (6.4) and the above computations

$$
\begin{aligned}
\|\mathscr{L} f\|_{L^{p}\left(\mu_{h}\right)} & =\left(\int_{0}^{\infty}|\mathscr{L} f|^{p} h d x\right)^{1 / p} \leq\left(\int_{0}^{\infty} \mathscr{L}\left(|f|^{p}\right) \widetilde{g} h d x\right)^{1 / p} \\
& \leq\|\widetilde{g}\|_{L^{p^{\prime}\left(\mu_{h}\right)}}^{1 / p} \sup _{0<g \in L^{p^{\prime}\left(\mu_{h}\right)}}\left(\|g\|_{L^{p^{\prime}\left(\mu_{h}\right)}}^{-1} \int_{0}^{\infty} \mathscr{L}\left(|f|^{p}\right) g h d x\right)^{1 / p} \\
& \leq\|\| K_{\mathscr{L}}\left\|_{1, \omega_{2}}\right\|_{L^{p}\left(\mu_{h}\right)}^{1 / p^{\prime}}\|f\|_{L^{p}\left(m_{p, h}\right)} .
\end{aligned}
$$

This, thanks to the order continuity of $L^{p}\left(m_{p, h}\right)$ and the continuity of $\mathscr{L}$, implies the desired bound.

Remark 6.8. We proved that the Laplace transform can be defined from the $p$-convex Banach function space $L^{p}\left(m_{p, h}\right)$ into the $p$-concave Banach function space $L^{p}\left(\mu_{h}\right)$. Then, the Laplace kernel operator $\mathscr{L}: L^{p}\left(m_{p, h}\right) \rightarrow L^{p}\left(\mu_{h}\right)$ is $q$-convex and also $p$-concave (see [66, p. 55]). On the other hand we know that it is a positive kernel operator (see [108, Th. 93.1(i)]). The Maurey-Rosenthal's Theorem allows us to provide two factorizations, the first one through $L^{p}\left(\mu_{h}\right)$ as $\mathscr{L}=M_{g} \circ R$ (which indeed is non relevant), and the second one through $L^{p}(v)$ as $\mathscr{L}=S \circ M_{f}$, where $v$ is a Rybakov measure for $m_{p, h}, R$ and $S$ are operators and $M_{g}$ and $M_{f}$ are positive multipliers of $g, f$ in $L^{0}\left(\mu_{h}\right)$ and $L^{0}(v)$ respectively, (see [23, Cors. 2 and 5]). This yields the factorization scheme of the Diagram 6.1 below, where the operators $R$ and $S$ are actually the compositions of the Laplace transform with the inverse of the multipliers, $M_{1 / g}$ and $M_{1 / f}$, respectively. See Remark 4.8. 


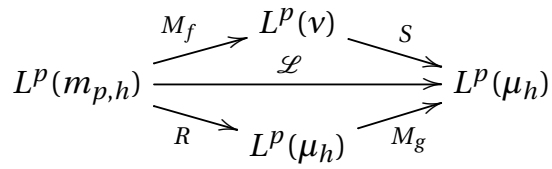

DiAGRAm 6.1. Maurey-Rosenthal factorization applied to the Laplace transform

\subsection{Factorization and optimal domain}

Let us recall that given a Banach function space $X$ over a finite measure, the $p$-th power space $X_{[p]}$ for $1 \leq p<\infty$ is a Banach function space whenever $X$ is $p$-convex with constant 1 . In the equality $\left(X_{[p]}\right)^{\prime}=\left(X^{p}\right)_{[p]}$, we note that $X^{p}$ is $p$-convex with constant 1 (see [12, Th. 5.1] or [97, Prop. 3.1]), then $\left(X^{p}\right)_{[p]}$ is a Banach function space as we can see in [79, Prop. 2.23(iii)]. However, if $X$ is not $p$-convex we cannot assure that $X_{[p]}$ is again a Banach function space, in consequence it may happens that $\left(X_{[p]}\right)^{\prime}=\{0\}$. This does not necessarily imply that $\left(X^{p}\right)_{[p]} \neq\left(X_{[p]}\right)^{\prime}$, e.g. $L^{p}(\mu)_{[q]}=L^{p / q}(\mu)$ has trivial dual when $p<q$ and $\mu$ is the Lebesgue measure. In this case $\left(L^{p}(\mu)\right)^{q}=\{0\}$ and so its $q$-th power space is also trivial. But if we assume that $X$ is $p$-convex with constant 1 we always have that $\left(X_{[p]}\right)^{\prime}=\left(X^{p}\right)_{[p]}$ isometrically for $p \in[1, \infty)$.

6.3.1. The $(p, q)$-th power factorization. Next result is the main tool of this section.

Theorem 6.9. Let $\mu$ and $\lambda$ be finite measures on $(\Omega, \Sigma)$. Let $1 \leq u, v<\infty$. Let $X(\mu)$ and $Y(\lambda)$ be order continuous Banach function spaces, so that $Y(\lambda)$ is Fatou and $v^{\prime}$-concave and $Y(\lambda)^{\prime}$ is order continuous. Assume that $X(\mu) \subseteq L^{u r}(\mu)$ and $Y(\lambda)^{\prime} \subseteq L^{\nu s}(\lambda)$ for some $1 \leq$ $s, r \leq \infty$. Let $K \in L^{1}(\lambda \otimes \mu)$ be a positive kernel and let $T:=T_{K}: X(\mu) \rightarrow Y(\lambda)$ the associated kernel operator. If

$$
\|\| K\left\|_{L^{s^{\prime}}(\lambda), \omega_{1}}\right\|_{L^{r^{\prime}(\mu)}}<\infty
$$

then $T$ is $(u, v)$-th power factorable.

Notice that this result can be applied to a broad class of Banach function spaces as Banach sequence spaces, Lebesgue, Lorentz, Nakano or Orlicz spaces among others.

Proof. By hypothesis \|\|$K\left\|_{L^{s^{\prime}}(\lambda), \omega_{1}}\right\|_{L^{r^{\prime}(\mu)}}<\infty$, and also $Y(\lambda)$ is $\nu^{\prime}$-concave, then $Y(\lambda)^{\prime}$ is $v$-convex. From Proposition 1.5(11), we know that $\left(\left(Y^{\prime}\right)_{[\nu]}\right)^{\prime}=\left(\left(Y^{\prime}\right)^{v}\right)_{[v]}$, the comments in the beginning of this section justify this equality. Thus for $h \in\left(\left(Y^{\prime}\right)_{[v]}\right)^{\prime}$, we can rewrite its norm in the following way

$$
\|h\|_{\left(\left(Y^{\prime}\right)_{[\nu]}\right)^{\prime}}=\|h\|_{\left(\left(Y^{\prime}\right)^{v}\right)_{[\nu]}}=\left\||h|^{1 / \nu}\right\|_{\left(Y^{\prime}\right)^{v}}^{v}=\left(\sup \left\{\left\|g|h|^{1 / \nu}\right\|_{L^{v}(\lambda)}: g \in B_{Y^{\prime}}\right\}\right)^{\nu} .
$$


This with the Hölder-Rogers inequality and $Y(\lambda)^{\prime} \subseteq L^{v s}$ justify the following inequalities for $f \in X(\mu)$

$$
\begin{aligned}
\|T f\|_{\left(\left(Y^{\prime}\right)_{[v]}\right)^{\prime}} & =\left(\sup _{g \in B_{Y^{\prime}}}\left\|g|T f|^{1 / v}\right\|_{L^{v}(\lambda)}\right)^{v}=\left(\sup _{g \in B_{Y^{\prime}}}\left(\left\||g|^{v}|T f|\right\|_{L^{1}(\lambda)}\right)^{1 / v}\right)^{v} \\
& \leq\left(\sup _{g \in B_{Y^{\prime}}}\left\||g|^{v}\right\|_{L^{s}(\lambda)}^{1 / v}\|T f\|_{L^{s^{\prime}}(\lambda)}^{1 / v}\right)^{v} \leq\left(\sup _{g \in B_{Y^{\prime}}}\|g\|_{L^{s v}(\lambda)}\right)^{v}\|T f\|_{L^{s^{\prime}}(\lambda)} \\
& \leq\left(\sup _{g \in B_{Y^{\prime}}} C\|g\|_{Y^{\prime}}\right)^{v}\left\||T f|^{s^{\prime}}\right\|_{L^{1}(\lambda)}^{1 / s^{\prime}} .
\end{aligned}
$$

By Minkowski's integral inequality (1.8), $\left\||T f|^{s^{\prime}}\right\|_{L^{1}(\lambda)}^{1 / s^{\prime}} \leq\||f|\| K\left\|_{L^{s^{\prime}(\lambda), \omega_{1}}}\right\|_{L^{1}(\mu)}$. Writing this in integral notation,

$$
\left(\int_{0}^{\infty}\left|\int_{0}^{\infty} K(x, y) f(y) d \mu(y)\right|^{s^{\prime}} d \lambda(x)\right)^{1 / s^{\prime}} \leq \int_{0}^{\infty}\left(\int_{0}^{\infty}|K(x, y) f(y)|^{s^{\prime}} d \lambda(x)\right)^{1 / s^{\prime}} d \mu(y) .
$$

As final step we use that $\|\cdot\|_{L^{r}(\mu)} \leq D\|\cdot\|_{X_{[u]}}$, which is true because $X(\mu)_{[u]} \subseteq L^{r}(\mu)$ since $X(\mu) \subseteq L^{u r}(\mu)$. So we have that

$$
\begin{aligned}
\|T f\|_{\left(\left(Y^{\prime}\right)[\nu]\right)^{\prime}} & \leq C^{v}\||f|\| K\left\|_{L^{s^{\prime}}(\lambda), \omega_{1}}\right\|_{L^{1}(\mu)} \\
& \leq C^{v}\|f\|_{L^{r}(\mu)}\|\| K\left\|_{L^{s^{\prime}}(\lambda), \omega_{1}}\right\|_{L^{r^{\prime}}(\mu)} \\
& \leq C^{v} D\|\| K\left\|_{L^{s^{\prime}}(\lambda), \omega_{1}}\right\|_{L^{r^{\prime}(\mu)}}\|f\|_{X_{[u]}} .
\end{aligned}
$$

Consequently there exists $T_{[u, v]}: X_{[u]} \rightarrow\left(Y_{[\nu]}^{\prime}\right)^{\prime}$ so that $T=j_{[v]}^{\prime} \circ T_{[u, v]} \circ i_{[u]}$. Theorem 4.4 gives that $T$ is $(u, v)$-th power factorable, and the proof is finished.

As a final application, we present the $(p, q)$-th power factorization for the Laplace transform. Recall from the previous section that $(p, q)$-th power factorability provides an extension operator that can restrict its range to a space embedded into the original codomain. Consider $X$ a Banach function space over $[0, \infty)$ with the Lebesgue measure. Let us show first a formula for the integral of a function $f \in X^{+}$with respect to the scalar measure $\langle m, g\rangle_{\mu}$, when $m: \Sigma \rightarrow Y(\mu)$ is defined by a positive kernel operator $T: X \rightarrow Y(\mu)$ and $g \in\left(Y(\mu)^{\prime}\right)^{+}$. By the barycentric formula (1.4) and Proposition 1.1(1) where have that

$$
\begin{aligned}
\int_{0}^{\infty} f(y) d\langle m, g\rangle_{\mu}(y) & =\left\langle\left(\int_{0}^{\infty} f d m\right)(y), g(y)\right\rangle_{\mu} \\
& =\int_{0}^{\infty} T(f)(y) g(y) d \mu(y) \\
& =\int_{0}^{\infty}\left(\int_{0}^{\infty} f(t) K(y, t) d t\right) g(y) d \mu(y)=\langle T f, g\rangle .
\end{aligned}
$$

Theorem 6.10. Let $1<q \leq 2 \leq p<\infty$, and let $0<h \in L^{1}[0, \infty) \cap L^{1}\left(\frac{d x}{x^{p}}\right)$ be a continuous function. Then $\mathscr{L}: L^{p}\left(m_{p, h}\right) \rightarrow L^{q}\left(\mu_{h}\right)$ is $(u, v)$-th power factorable for $u \in[p / 2, p)$ and $v \in\left[1, q^{\prime} / 2\right]$. 
Proof. We will see that the Laplace transform $\mathscr{L}: L^{p}\left(m_{p, h}\right) \rightarrow L^{q}\left(\mu_{h}\right)$ satisfies the hypothesis of Theorem 6.9. The following argument says that if $g>0 \mu_{h}$-almost everywhere and belongs to $L^{\infty}\left(\mu_{h}\right) \subseteq L^{q^{\prime}}\left(\mu_{h}\right)$, then it defines a Rybakov measure for the vector measure $m_{p, h}$. Let $A \in \Sigma$ be such that

$$
m_{p, h}(A)=\mathscr{L}\left(\chi_{A}\right)=\int_{0}^{\infty} e^{-x y} \chi_{A}(y) d y=0
$$

a.e., since $e^{-x y}>0$ for all $x, y \in \mathbb{R}$, necessarily $\chi_{A}=0$ almost everywhere, then

$$
\left\langle m_{p, h}, g\right\rangle_{\mu_{h}}(A)=\int_{0}^{\infty} g \mathscr{L}\left(\chi_{A}\right) h d x=0 .
$$

The converse is analogous.

Now we find, for a suitable $1 \leq s<\infty$, a bound for the $s$-integral of $K_{\mathscr{L}}$ with respect to $\left|\left\langle m_{p, h}, g\right\rangle\right|=\left\langle m_{p, h}, g\right\rangle$, where $0<g \in L^{\infty}\left(\mu_{h}\right)$. Since $p \geq 2$, by Proposition 6.4(3) we can choose $s \in[2, \infty)$ such that $L^{s}[0, \infty) \subseteq L^{p}\left(m_{p, h}\right)$. Recall that $m_{p, h}$ is a positive measure and that $e^{-a y} \in L^{s}[0, \infty) \subseteq L^{p}\left(m_{p, h}\right)$. Hence, using (6.6) for $T=\mathscr{L}$ and $K(x, y)=e^{x y}$ for every $x, y>0$, we obtain the following

$$
\int_{0}^{\infty} e^{-x y s} d\left\langle m_{p, h}, g\right\rangle_{\mu_{h}}(y)=\int_{0}^{\infty}\left(\int_{0}^{\infty} e^{-x t s} e^{-y t} d t\right) g(y) h(y) d y=\int_{0}^{\infty} \frac{g(y)}{x s+y} h(y) d y .
$$

Since $s \geq 2$, let us now assume that $s=r^{\prime}$ for some $1<r \leq 2$, i.e. $\frac{1}{r}+\frac{1}{s}=1$, thus $r \leq r^{\prime}$. Then the following computation is straightforward using the Minkowski's integral inequality and the calculation above with $g:=\chi_{[0, \infty)}$ (observe that $g \in L^{\infty}\left(\mu_{h}\right)$ ).

$$
\begin{aligned}
\|\| K_{\mathscr{L}}\left\|_{L^{r}\left(\mu_{h}\right), \omega_{1}}\right\|_{L^{r^{\prime}}\left(\left|\left\langle m_{p, h}, g\right\rangle\right|\right)} & \\
& =\left(\int_{0}^{\infty}\left(\int_{0}^{\infty} e^{-x y r} h(x) d x\right)^{r^{\prime} / r} d\left\langle m_{p, h}, \chi_{[0, \infty)}\right\rangle(y)\right)^{1 / r^{\prime}} \\
& \leq\left(\int_{0}^{\infty}\left(\int_{0}^{\infty} e^{-x y r^{\prime}} d\left\langle m_{p, h}, \chi_{[0, \infty)}\right\rangle(y)\right)^{r / r^{\prime}} h(x) d x\right)^{1 / r} \\
& =\left(\int_{0}^{\infty}\left(\int_{0}^{\infty} \frac{1}{x r^{\prime}+y} h(y) d y\right)^{r / r^{\prime}} h(x) d x\right)^{1 / r} \\
& \leq\left(\int_{0}^{\infty}\left(\int_{0}^{\infty} \frac{h(y)}{y} d y\right)^{r / r^{\prime}} h(x) d x\right)^{1 / r} \\
& \leq\left(\int_{0}^{\infty} \frac{h(y)}{y} d y\right)^{1 / r^{\prime}}\left(\int_{0}^{\infty} h(x) d x\right)^{1 / r}<\infty
\end{aligned}
$$

by hypothesis $h \in L^{1}[0, \infty) \cap L^{1}\left(\frac{d x}{x^{p}}\right)$, thus it satisfies (6.1), i.e.

$$
\int_{0}^{\infty} \frac{h(y)}{y} d y=\left\|\frac{1}{y}\right\|_{L^{1}\left(\mu_{h}\right)} \leq C\left\|\frac{1}{y}\right\|_{L^{p}\left(\mu_{h}\right)}=C\left(\int_{0}^{\infty} \frac{h(y)}{y^{p}} d y\right)^{1 / p}<\infty .
$$

Then we conclude that condition (6.5) is satisfied. In addition notice that

$$
L^{p}\left(m_{p, h}\right) \subseteq L^{p}\left(\left|\left\langle m_{p, h}, g\right\rangle\right|\right) \subseteq L^{(a / r) r}\left(\left|\left\langle m_{p, h}, g\right\rangle\right|\right),
$$


for $a \in[1, p]$. Analogously

$$
L^{q^{\prime}}\left(\mu_{h}\right) \subseteq L^{\left(b / r^{\prime}\right) r^{\prime}}\left(\mu_{h}\right)
$$

for $b \in\left[1, q^{\prime}\right]$. Moreover $L^{p}\left(m_{p, h}\right), L^{q}\left(\mu_{h}\right)$ and $L^{q^{\prime}}\left(\mu_{h}\right)$ are order continuous and $L^{q}\left(\mu_{h}\right)$ is Fatou. Also $L^{q}\left(\mu_{h}\right)$ is $q^{\prime}$ concave since $q \leq q^{\prime}$ (recall that $q \in(1,2]$ ), and we can apply Theorem 6.9 , taking $X:=L^{p}\left(m_{p, h}\right)$ and $Y:=L^{q}\left(\mu_{h}\right)$. So $\mathscr{L}$ is $\left(a / r, b / r^{\prime}\right)$-th power factorable. We have assumed that $r \leq r^{\prime}$, hence $r^{\prime} \in[2, \infty)$ and $r \in(1,2]$. In consequence $a / r \in[p / 2, p)$ and $b / r^{\prime} \in\left(0, q^{\prime} / 2\right]$, recall that for a well defined factorization it is necessary that $b / r^{\prime} \geq 1$ and $a / r \geq 1$, which is truth thanks to that $1<q \leq 2 \leq p<\infty$. Therefore we can conclude that $T$ is $(u, v)$-th power factorable for $u \in[p / 2, p)$ and $v \in\left[1, q^{\prime} / 2\right]$.

\subsubsection{Final remarks and example.}

Remark 6.11. Thanks to the symmetry of the Laplace kernel, the adjoint of the Laplace transform from a Banach function space $X$ into another one $Y$ is the Laplace transform too but from $Y^{\prime}$ into $X^{\prime}$.

Remark 6.12. Observe that Theorem 6.10 do not include the basic cases $L^{1}\left(m_{p, h}\right)$ and $L^{1}\left(\mu_{h}\right)$, since their duals are not order continuous and so Theorem 6.9 does not work. However, since the measures are finite, we always have the standard embeddings of the spaces of $p$-integrable and $q$-integrable functions into spaces of integrable functions. Thus, when $\mathscr{L}$ is $(u, v)$-th power factorable - for $1<q \leq 2 \leq p<\infty, u \in[p / 2, p)$ and $\left.v \in\left[1, q^{\prime} / 2\right]\right)-$ we have the following diagram

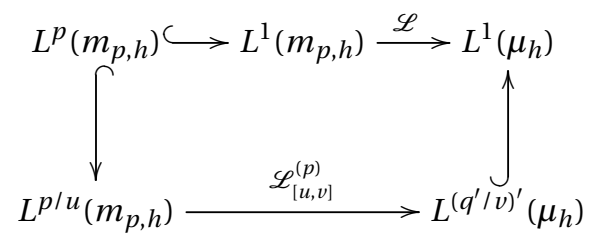

DIAGRAM 6.2. $(p, q)$-th power factorization for the Laplace transform

where $\mathscr{L}^{(p)}: L^{p}\left(m_{p, h}\right) \rightarrow L^{q}\left(\mu_{h}\right)$ is the restriction of $\mathscr{L}$.

Remark 6.13. In Theorem 6.10 we also have proved that $\mathscr{L}^{\prime}$ is a Hille-Tamarkin operator (see Section 1.2.2) from $L^{s}\left(\mu_{h}\right)$ into $L^{s}(|\langle m, g\rangle|)$ for a suitable $2 \leq s \leq \infty$, see equation (6.7). Then, by [94, Th. 2.3], it is an order continuous compact operator, but in addition, thanks to the Schauder's Theorem (see [93]), its adjoint operator is also compact, we can conclude that the Laplace transform is compact as operator from $L^{r}(|\langle m, g\rangle|)$ into $L^{r}\left(\mu_{h}\right)$ for some $r=s^{\prime} \in[1,2]$.

Remark 6.14. Once we have defined the Laplace transform between spaces of integrable functions $L^{p^{\prime}}(\lambda)$ and $L^{q^{\prime}}(\eta)$ with respect to positive, finite scalar measures, another point of view can be used. Observe that considering the bilinear form associated with the Laplace transform we can set that

$$
|\langle\mathscr{L}(f), g\rangle| \leq C\|f\|_{L^{p}(\lambda)}\|g\|_{L^{q^{\prime}(\eta)}}
$$


for suitable positive and finite measures $\lambda$ and $\eta$, i.e. for suitable $u, v \geq 1$ the Laplace transform is $(u, v)$-th power factorable (see Theorem 4.7 and Proposition 5.13). The techniques used in [50] would be applied in order to obtain this type of inequalities for CalderónZygmund operators. In consequence, results related with the $(p, q)$-th power factorization (see Section 5.2) would be obtained.

Example 6.15 (A factorization for the Laplace transform). To finish this thesis we present as an example a concrete factorization for the Laplace transform. This exercise will allow us to realize how far the techniques presented here can be used. For example, we may define the Laplace transform between Hilbert spaces of functions or spaces of integrable functions, giving the concrete finite measures where they are defined.

Let $1<q \leq 2 \leq p<\infty$ and let the function $h$ of the Example 6.1, i.e. $h(x):=x^{k} e^{-x}$ for $k \in[p, \infty) \cap \mathbb{Z}$, which is continuous and it is easy to check that $0<h \in L^{1}[0, \infty) \cap L^{1}\left(\frac{d x}{x^{p}}\right)$. In that case Theorem 6.10 says that $\mathscr{L}: L^{p}\left(m_{p, h}\right) \rightarrow L^{q}\left(\mu_{h}\right)$ is $(u, v)$-th power factorable for $u \in[p / 2, p)$ and $v \in\left[1, q^{\prime} / 2\right]$. On one hand $p / u \in(1,2],\left(q^{\prime} / v\right)^{\prime} \in[q, 2]$ and $q \leq 2$, hence we can choose $u_{0} \in[p / 2, p)$ and $v_{0} \in\left[1, q^{\prime} / 2\right]$ so that $p / u_{0} \leq\left(q^{\prime} / v_{0}\right)^{\prime}$, then $L^{\left(q^{\prime} / v_{0}\right)^{\prime}}\left(\mu_{h}\right)$ is $p / u_{0}$ concave. On the other hand, since the kernel of the Laplace transform is positive, by [66, Th. 1.d.9], it follows that $I_{m_{p, h}}^{\left(p / u_{0}\right)}=\mathscr{L}$ is $p / u_{0}$-concave. Therefore [79, Rem. 6.42(i)and(ii)] implies that we have an extension of $\mathscr{L}$ to $L^{p / u}\left(\left\langle m_{p, h}, g\right\rangle\right)$, i.e. we obtain the following commutative diagram for adequate $u_{0}, v_{0}$ and $g \in L^{q^{\prime}}\left(\mu_{h}\right)$

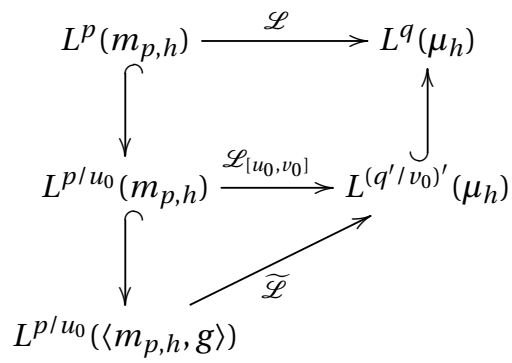

DIAGRAM 6.3. Example of $(p, q)$-th power factorization for the Laplace transform

Let $A$ be a countable union of disjoint open intervals, i.e. $A=\bigcup_{i=1}^{\infty}\left(a_{i}, b_{i}\right)$, then

$$
\begin{aligned}
m_{p, h}(A) & =\mathscr{L}\left(\chi_{A}\right)=\int_{A} e^{-x y} d y \\
& =\int_{\bigcup_{i=1}^{\infty}\left[a_{i}, b_{i}\right)} e^{-x y} d y=\sum_{i=1}^{\infty} \int_{a_{i}}^{b_{i}} e^{-x y} d y=\sum_{i=1}^{\infty} \frac{e^{-x a_{i}}-e^{-x b_{i}}}{x} .
\end{aligned}
$$

Now we need a Rybakov measure for $m_{p, h}$, namely $v$ which is defined by a function belonging to $L^{p}\left(\mu_{h}\right)^{\prime} \subseteq\left(L^{p^{\prime}}\left(\mu_{h}\right)\right)_{[q]}$. Take for instance $g(x):=x$. Proposition 6.4(1) ensure us that $\left\langle m_{p, h}, g\right\rangle_{\mu_{h}}$ is a Rybakov measure, since it has the same null sets as $m_{h, p}$. Recall that 
$h(x):=x^{k} e^{-x}$ for $k \in[p, \infty) \cap \mathbb{Z}$, thus

$$
\begin{aligned}
v(A) & :=\left\langle m_{p, h}, g\right\rangle_{\mu_{h}}(A)=\sum_{i=1}^{\infty} \int_{0}^{\infty} x \frac{e^{-x a_{i}}-e^{-x b_{i}}}{x} h(x) d x \\
& =\sum_{i=1}^{\infty} \int_{0}^{\infty} e^{-x a_{i}} h(x) d x-\int_{0}^{\infty} e^{-x b_{i}} h(x) d x \\
& =\sum_{i=1}^{\infty} \int_{0}^{\infty} x^{k} e^{-x\left(a_{i}+1\right)} d x-\int_{0}^{\infty} x^{k} e^{-x\left(b_{i}+1\right)} \\
& =\sum_{i=1}^{\infty}\left(\mathscr{L}\left(x^{k}\right)\right)\left(a_{i}+1\right)-\left(\mathscr{L}\left(x^{k}\right)\right)\left(b_{i}+1\right) .
\end{aligned}
$$

Now we can write that

$$
v(A)=k ! \sum_{i=1}^{\infty} \frac{1}{\left(a_{i}+1\right)^{k+1}}-\frac{1}{\left(b_{i}+1\right)^{k+1}} .
$$

Observe that this measure acts as a "contraction" of any set in $[0, \infty)$ to a set in the interval $[0,1]$ of finite Lebesgue measure, so it is easy to realize that such series really converges.

At the end, by Diagram 6.3 and the comments above it, we have obtained that

$$
\mathscr{L}: L^{r}(v) \rightarrow L^{s}\left(\frac{x^{k}}{e^{x}} d x\right),
$$

for $1<r \leq s \leq 2$, where $L^{r}\left(m_{p, h}\right) \subseteq L^{r}(v)$. Taking the adjoint operator we obtain the Laplace transform from the space $L^{s^{\prime}}\left(\frac{x^{k}}{e^{x}} d x\right)$ into $L^{r^{\prime}}(v) \subseteq\left(L^{r}\left(m_{p, h}\right)\right)^{\prime}$ where $r \leq 2 \leq s^{\prime} \leq r^{\prime}<\infty$. This result is related to Theorem 1.9, however the techniques used here are quite different. Finally observe that thanks to the $(u, v)$-th power factorization, we have obtained a set of spaces of integrable functions with respect to a concrete Rybakov measure where the Laplace transform is well defined. For example if $r=s=2$, then we obtain a Laplace transform between Hilbert spaces. This factorization has been studied in [88]. 



\section{BIBLIOGRAPHY}

[1] Abramovich, Y.A. and Aliprantis, C.D., An Invitation to Operator Theory, Graduate Studies in Mathematics, vol. 50, American Mathematical Society, Providence, 2002.

[2] Aliprantis, C.D. and Burkinshaw, O, Locally Solid Riesz Spaces, Academic Press, New York, 1978.

[3] _ Positive Operators, Springer, Berlin, 2006.

[4] Anderson, K.F. and Heinig, H.P., Weighted norm inequalities for certain integral operators, SIAM Journal of Mathematical Analysis 14 (1983), 833-844.

[5] Arendt, W., Batty, C.J.K., Hieber, M. and Neubrander, F., Vector-valued Laplace Transforms and Cauchy Problems, Birkhäuser, Basel, 2001.

[6] Aronzajn, N. and Szeptycki, P., On general integral transformations, Mathematische Annalen 163 (1966), 127-154.

[7] Bäumer, B. and Neubrander, F., Laplace transform methods for evolution equations, Conferencenze del Seminario di Matematica dell'Università di Bari 259 (1995), 2760.

[8] Bennet, C. and Sharpley, R., Interpolation of Operators, Academic Press, Orlando, 1988.

[9] Bergh. J. and Löfström, Interpolation Spaces. an Introduction, Springer, Berlin, 1976.

[10] Bloom, S., Hardy integral estimates for the Laplace transform, Proceedings of the American Mathematical Society 116 (1992), no. 2, 417-426.

[11] Bradley, J.S., Hardy inequalities with mixed norms, Canadian Mathematical Bulletin 21 (1978), no. 4, 405-408.

[12] Calabuig, J.M., Delgado, O. and Sánchez-Pérez, E.A., Generalized perfect spaces, Indagationes Mathematicae 19 (2008), no. 3, 359-378.

[13] __ Factorizing operators on Banach function spaces through spaces of multiplication operators, Journal of Mathematical Analysis and Applications 364 (2010), 88-103.

[14] Calderón, A.P., Intermediate spaces and interpolation, the complex method, Studia Mathematica 24 (1964), no. 2, 113-190.

[15] Curbera, G.P., The space of integrable functions with respect to a vector measure, Ph.D. thesis, University of Sevilla, Spain, 1992.

[16] _ When $L^{1}$ of a vector measure is an AL-space, Pacific Journal of Mathematics 162 (1994), no. 2, 287-303.

[17] Curbera, G.P. and Ricker, W.J., Optimal domains for kernel operators via interpolation, Mathematische Nachrichten 244 (2002), 47-63. 
[18] __ Optimal domains for the kernel operator associated with Sobolev's inequality, Studia Mathematica 158 and 170 (2003 and 2005), 131-152 and 217-218.

[19] _ Banach lattices with the Fatou property and optimal domains of kernel operators, Indagationes Mathematicae (N.S.) 170 (2006), 187-204.

[20] _ Can optimal rearrangement invariant Sobolev imbeddings be further extended?, Indiana University Mathematics Journal 56 (2007), 1479-1497.

[21] _ Compactness properties of Sobolev imbeddings for rearrangement invariant norms, Transactions of the American Mathematical Society 359 (2007), 1471-1484.

[22] Cwikel, M., Milman, M. and Sagher, Y., Complex interpolation of some quasi-Banach spaces, Journal of Functional Analysis 65 (1986), 339-347.

[23] Defant, A., Variants of the Maurey-Rosenthal theorem for quasi Köthe function spaces, Positivity 5 (2001), 153-175.

[24] Defant, A. and Floret, K., Tensor norms and operator ideals, Mathematic Studies, vol. 176, North-Holland, Amsterdam, 1993.

[25] Defant, A. and Sánchez-Pérez, E.A., Maurey-Rosenthal factorization of positive operators and convexity, Journal of Mathematical Analysis and Applications 297 (2004), 771-790.

[26] del Campo, R., Fernández, A., Mayoral, F., Naranjo, F. and Sánchez-Pérez, E.A., Interpolation of vector measures, Acta Mathematica Sinica 27 (2011), no. 1, 119-134.

[27] Delgado, O., Futher developments on $L^{1}$ of a vector measure, Ph.D. thesis, University of Sevilla, Spain, 2004.

[28] _ Optimal domains for kernel operators on $[0, \infty) \times[0, \infty)$, Studia Mathematica 174 (2006), no. 2, 131-145.

[29] _ Rearrangement invariant optimal domains for monotone kernel operators, Operator Theory: Advances and Applications 201 (2009), 149-158.

[30] Delgado, O. and Soria, J., Optimal domain for the Hardy operator, Journal of Functional Analysis 244 (2007), 119-133.

[31] Diestel, J. and Uhl Jr., J.J., Vector Measures, American Mathematical Society, Providence, 1977.

[32] Dinculeanu, N., Vector Measures, Pergamon Press, New York, 1967.

[33] Dunford, N. and Schwartz, J.T., Linear Operators, PartI: General Theory, WilleyInterscience Publishers, New York, 1958.

[34] Edmunds, D., Kerman, R. and Pick, L., Optimal Sobolev imbeddings involving rearrangement-invariant quasi-norms, Journal of Functional Analysis 170 (2000), 307-355.

[35] Fabian, M., Habala, P., Hájek, P., Montesinos Santalucía, V., Pelant, J. and Zizler, V., Functional Analysis and Infinite-Dimensional Geometry, Springer, New York, 2001.

[36] Fernández, A., Mayoral, F. and Naranjo, F., Real interpolation method on spaces of scalar integrable functions with respect to a vector measure, Journal of Mathematical Analysis and Applications 376 (2011), 203-211. 
[37] Fernández, A., Mayoral, F., Naranjo, F., Sáez, C. and Sánchez-Pérez, E.A., Vector measure Maurey-Rosenthal-type factorizations and $\ell$-sums of $L^{1}$-spaces, Journal of Functional Analysis 220 (2005), no. 2, 460-485.

[38] __ Spaces of $p$-integrable functions with respect to a vector measure, Positivity 10 (2006), 1-16.

[39] __ Spaces of integrable functions with respect to a vector measure and factorizations through $L^{p}$ and Hilbert spaces, Journal of Mathematical Analysis and Applications 330 (2007), 1249-1263.

[40] Fernández, A., Mayoral, F., Naranjo, F. and Sánchez-Pérez, E.A., Complex interpolation of spaces of integrable functions with respect to a vector measure, Collectanea Mathematica 62 (2010), no. 2, 241-252.

[41] Galdames Bravo, O., Condicions per a la factorització per la p-èssima potència de l'adjunt d'un operador d'espais de Banach de funcions $i$ algunes aplicacions, M.Sc. thesis, University of Valencia, Spain, 2011.

[42] García Cuerva, J., Factorization of operators and weighted norm inequalities, Nonlinear Analysis, Function Spaces and Applications (Proceedings of the Spring School held in Roudnice nad Labem), Teubner, Leipzig (Krbec, M., Kufner, A., Opic, B. and Rákosník, J., eds.), Teubner Texte zur Mathematik, Band 119, vol. 4, B.G. Teubner Verlagsgesellschaft, Leipzig., 1990, pp. 5-41.

[43] García Cuerva, J. and Rubio de Francia, J.L., Weighted Norm Inequalities and Related Topics, Mathematics Studies, vol. 166, North-Holland, Amsterdam, 1985.

[44] Gómez, M. and Milman, M., Complex interpolation of $H^{p}$-spaces on product domains, Annali di Matematica Pura ed Applicata 155 (1989), 103-115.

[45] Halmos, P.R., Measure Theory, Springer, New York, 1974.

[46] _ Bounded Integral Operators on $L^{2}$-spaces, Springer, New York, 1978.

[47] Hardy, G.H., Remarks in addition to Dr. Widder's note on inequalities, Journal of the London Mathematical Society 4 (1929), 199-102.

[48] _ The constants of certain inequalities, Journal of the London Mathematical Society 8 (1933), 114-119.

[49] Hardy, G.H., Littlewood, J.E. and Pólya, G., Inequalities, Cambridge University Press, Cambridge, 1952.

[50] Hart, J., Bilinear square functions and vector-valued Calderón-Zygmund operators, Journal of Fourier Analysis and Applications 18 (2012), no. 6, 1291-1313.

[51] Hartley, R.V.L., A more symmetrical Fourier analysis applied to transmission problems, Proceedings of Institute of Engineers 30 (1942), no. 3, 144-150.

[52] Howard, R. and Schep, A.R., Norm of positive operators on $L^{p}$ spaces, Proceedings of the American Mathematical Society 109 (1990), no. 1, 135-146.

[53] Juan Blanco, M.A., Vector Measures on $\delta$-rings and Representation Theorems of Banach Lattices, Ph.D. thesis, Polytechnic University of Valencia, Spain, 2011.

[54] Kalton, N. and Mitrea, M., Stability of Fredholm properties on interpolation scales of quasi-Banach spaces and applications, Transactions of the American Mathematical Society 350 (1998), 3837-3901. 
[55] Kalton, N.J., Quasi-Banach spaces, Handbook of the Geometry of Banach Spaces (Johnson, W.B. and Lindenstrauss, J., eds.), vol. 2, Elsevier Science B.V., Amsterdam, 2003, pp. 1099-1130.

[56] Kalton, N.J., Peck, N.T. and Roberts, J.W., An F-space Sampler, Cambridge University Press, Cambridge, 1984.

[57] Khudak, I., On the regulatization of solutions of integral equations of the first kind, USSR Computation Mathematics and Mathematical Physics 6 (1966), no. 4, 217-221.

[58] Kokilashvili, V.M. and Krbec, M., Weighted Inequalities in Lorentz and Orlicz spaces, World Scientific Publishing Co. Pte. Ltd., Singapore, 1991.

[59] Kolwicz, P., Lésnik, K. and Maligranda, L., Pointwise multipliers of CalderónLozanowskil, arXiv:1206.1860v1 [math.FA] 8 Jun 2012.

[60] Krasnosel'skiı̆, M.A. and Rutickiı̆, Ya.B., Convex Functions and Orlicz Spaces, (translation), P. Noordhoff, Ltd., Groningen, 1961.

[61] Kreĭn, S.G., Petunin, Yu.I. and Semenov, E.M., Interpolation of linear operators, (english translation), Translations of Mathematical Monographs, vol. 54, American Mathematical Society, Providence, 1985.

[62] Krivine, J.L., Théorémes de factorisation dans les espaces reticulés, Seminaire MaureySchwartz 1973-74, Exposés 22-23, École Polytechnique, Paris.

[63] Kryczka, A and Prus, S., Measure of weak noncompactness under complex interpolation, Studia Mathematica 147 (2001), no. 1, 89-102.

[64] Kwapień, S., Isomorphic characterizations of inner product spaces by orthogonal series with vector valued coefficients, Studia Mathematica 44 (1972), 583-595.

[65] Labuda, I. and Szeptycki, P., Extended domains of some integral operators with rapidly oscillating kernels, Nederland Akad. Wetensch. Indagationes Mathematicae 48 (1986), 87-98.

[66] Lindenstrauss, J. and Tzafriri, L., Classical Banach Spaces II, Springer, Berlin, 1979.

[67] Lozanovskiū, G.Ya., On some Banach lattices, Siberian Mathematical Journal 10 (1969), no. 3, 584-599.

[68] Luxemburg, W.A.J., Banach Function Spaces, Ph.D. thesis, Delft Institute of Technology, Assen (Netherland), 1955.

[69] Luxemburg, W.A.J. and Zaanen, A.C., Riesz spaces I, North-Holland, Amsterdam, 1971.

[70] Maligranda, L., The K-functions of p-convexifications, Positivity 10.1007/s11117012-0200-x (2012).

[71] Maligranda, L. and Persson, L.E., Generalized duality of some Banach function spaces, Indagationes Mathematicae 51 (1989), no. 3, 323-338.

[72] Martis in Biddau, S., Studio della trasformazione di Laplace e della sua inversa dal punto di vista dei funzionali amalitici, Rendiconti del Circolo Matematico di Palermo 57 (1933), 1-70.

[73] Meyer-Nieberg, P., Banach Lattices, Springer, Berlin, 1991.

[74] Miyadera, I., On the representation theorem by Laplace transformation of vectorvalued functions, Tohoku Mathematical Journal 8 (1956), 170-180. 
[75] Mockenhaupt, G. and Ricker, W.J., Optimal extension of the Hausdorff-Young inequality, Journal für die reine angewandte Mathematik 620 (2008), 195-211.

[76] Mugnolo, D. and Nittka, R., Properties of representation of operators acting between spaces of vector-valued functions, Positivity 15 (2011), no. 1, 135-154.

[77] Muraz, G. and Szeptycki, P., Domains of trigonometric transforms, Rocky Mountain Journal Mathematics 26 (1996), 1517-1527.

[78] Musial, K., The weak Radon-Nikodým property in Banach spaces, Studia Mathematica 64 (1979), 151-173.

[79] Okada, S., Ricker, W.J. and Sánchez-Pérez, E.A., Optimal Domain and Integral Extension of Operators, Acting in Function Spaces, Operator Theory: Advances and Applications, vol. 180, Birkhäuser, Basel, 2008.

[80] Okada, S. and Ricker, W.J., Optimal domains and integral representations of convolution operators in $L^{p}(G)$, Integral Equations and Operator Theory 48 (2004), 525-546.

[81] __ Optimal domains and integral representations of $L^{p}(G)$-valued convolution operators via measures, Mathematische Nachrichten 280 (2007), 423-436.

[82] Okada, S., Ricker, W.J. and Rodríguez-Piazza, L., Compactness of the integration operator associated with a vector measure, Studia Mathematica 150 (2002), 133-149.

[83] Palazuelos, C., Sánchez-Pérez, E.A. and Tradacete, P., Maurey-Rosenthal factorization of $p$-summing operators and Dodds-Fremlin domination, Journal of Operator Theory 68 (2012), no. 1, 205-222.

[84] Peetre, J., Locally analitically pseudo-convex topological vector spaces, Studia Mathematica 73 (1982), 253-262.

[85] Persson, L.E., Stepanov, V.D. and Ushakova, E.P., On integral operators with monotone kernel, Doklady Mathematics 403 (2005), no. 1, 11-14.

[86] Pick, L., Optimal Sobolev embeddings, Nonlinear Analysis, Function Spaces and Applications (Krbec, M. and Kufner, A., eds.), vol. 6, Czech Academy of Science, Mathematical Institute, Praha, 1999, pp. 156-199.

[87] Pietsch, A., Operator Ideals, North-Holland, New York, 1980.

[88] Pisier, G., Factorization of Linear Operators and Geometry of Banach Spaces, American Mathematical Society, Providence, 1987.

[89] Post, E.L., Generalized differentiation, Transactions of the American Mathematical Society 32 (1930), 723-781.

[90] Rao, M.M. and Ren, Z.D., Applications of Orlicz Spaces, Marcel Dekker, Inc., New York, 2002.

[91] Riviere, N.M., Interpolation Theory in s-Banach Spaces, Ph.D. thesis, University of Chicago, Chicago. I11, 1966.

[92] Rolewicz, S., Metric Linear Spaces, Interscience Tracts in Pure and Applied Mathematics, PWN-Polish Science Publisher, Reidel and Dordretcht, 1984.

[93] Schauder, J., Uber lineare, vollstetige funktionaloperationen, Studia Mathematica 2 (1930), 183-196.

[94] Schep, A.R., Compactness properties of Carleman and Hille-Tamarkin operators, Canadian Journal of Mathematics 37 (1985), no. 5, 921-933. 
[95] __ Minkowski's integral inequality for function norms, Operator Theory in Function Spaces and Banach Lattices (Huijsmans, C.B., Kaashoek, M.A., Luxemburg, W.A.J. and de Pagter, B., eds.), Operator Theory: Advances and Applications, vol. 75, Birkhäuser, Basel, 1995, pp. 299-308.

[96] __ When is the optimal domain of a positive linear operator a weighted $L^{1}$-space?, Operator Theory: Advances and Applications 201 (2009), 361-369.

[97] __ Products and factors of Banach function spaces, Positivity 14 (2010), 301-319.

[98] Schwartz, H. U., Banach Lattices and Operators, B.G. Teubner Verlagsgesellschaft, Leipzig, 1984.

[99] Stepanov, V.D. and Ushakova, E.P., Weighted estimates for the integral operators with monotone kernel on half-axis, Siberian Mathematical Journal 45 (2004), no. 6, 11241134.

[100] Szeptycki, P., Notes on integral transformations, Dissertationes Mathematicae 231 (1984), 48.

[101] _ Extended domains of some integral operators, Rocky Mountain Journal in Mathematics 22 (1992), 393-404.

[102] Tabacco Vignati, A., Complex interpolation for families of quasi-Banach spaces, Indiana University Mathematics Journal 37 (1988), no. 1, 1-21.

[103] Ushakova, E.P., Estimates for the singular values of Stieltjes type transforms, Siberian Mathematical Journal 52 (2011), no. 1, 159-166.

[104] Viselter, A., Generalized Widder theorem via fractional moments, Journal of Functional Analysis 256 (2009), 594-602.

[105] Widder, D.V., A classification of generating functions, Transactions of the American Mathematical Society 39 (1936), 244-298.

[106] Wojtaszczyk, P., Banach Spaces for Analysts, Cambridge University Press, Cambridge, 1991.

[107] Zaanen, A.C., Integration, 2nd rev. ed., North-Holland, Amsterdam, 1967.

[108] __ Riesz Spaces II, North-Holland, Amsterdam, 1983.

[109] Zaidman, S., On the representation of vector-valued functions by Laplace transforms, Duke Mathematical Journal 26 (1959), 189-191.

[110] Zwillinger, D., Standard Mathematical Tables and Formulae, 31st ed., Chapman \& Hall/CRC, New York, 2003. 


\section{LIST OF DIAGRAMS}

1.1 Definition of $p$-th power factorable operator 8

$1.2 p$-th power factorization versus factorization through optimal domains 9

1.3 Factorizaton through $L^{p}$-spaces for $T: L^{p}(n) \rightarrow\left(L^{q}(m)\right)^{\prime} \quad 10$

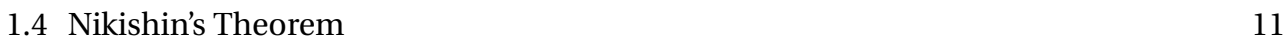

2.1 Köthe $p$-adjoint operator 16

2.2 Context diagram for the Köthe $p$-dual space and Köthe $p$-adjoint operator $\quad 18$

3.1 Factorization through the inclusion/quotient map 27

3.2 Optimal domain for the adjoint operator 30

3.3 Characterization for the optimal range 32

3.4 Optimal range for the Volterra operator 34

3.5 Factorization through an $L^{s}$-space for the adjoint of the integration map $\left(I_{m_{V^{\prime}}}\right)^{\prime} \quad 35$

4.1 Duality for the $p$-th power space 38

4.2 Characterization I for $(p, q)$-th power factorable operators 39

4.3 Characterization II for $(p, q)$-th power factorable operators 40

4.4 Maurey-Rosenthal factorization applied to a $(p, q)$-th power factorable operator 42

4.5 Square factorization through $L^{p}\left(m_{T}\right)$ and $L^{q}\left(m_{T^{\prime}}\right)^{\prime} \quad 42$

4.6 Factorization through Banach function spaces defined over finite measures $\quad 45$

$4.7(p, q)$-th power factorization for non finite measure based spaces 46

5.1 Applying of the optimal domain theorem to the interpolation operator 51

$5.2 p_{\theta}$-th power factorization for the interpolated operator $T_{\theta}$

5.3 Interpolation for extensions of $(p, q)$-th power factorable operators 55

5.4 Factorization through $L^{p}$-spaces for kernel operators 59

6.1 Maurey-Rosenthal factorization applied to the Laplace transform 70

$6.2(p, q)$-th power factorization for the Laplace transform 73

6.3 Example of $(p, q)$-th power factorization for the Laplace transform 74 



\section{INDEX}

absolute kernel, 60

absolute value, 2

absolutely continuous, 26

adjoint operator, 3

kernel, 11

admissible operator, 48

almost $L^{p}$-space, 29

AM-space, 20, 21, 29

Aoki-Rolewicz's Theorem, 7

associated integration map, 26, 29, 34

associated vector measure, 5, 25, 32, 63

Banach function space, 2

Banach lattice, 2

barycentric equality, 4

Borel set, 2

Calderón complex interpolation, 48

Calderón-Lozanowskiı̌ product space, 48

Carleman operators, 11

compatible pair, 47

$q$-concave

operator, 4

space, 4

complete, 1

$p$-convex

operator, 4

space, 4

Convolution operator, 33

cotype, 4,20

$\mu$-determined operator, 5, 25, 29, 47
Egoroff's Theorem, 65

Fatou property, 3

finite variation, 1

Hartley transform, 60

Hille-Tamarkin operator, 57, 59, 64, 73

inclusion/quotient map, 2, 27

integrable

weakly, 4

$m$-integrable, 4

$p$-integrable with respect to a vector

measure, 5

intermediate space, 47

Köthe $p$-adjoint operator, 16

Köthe $p$-dual space, 13

Köthe adjoint operator, 3

kernel function, 11

kernel operator, 11

kernel operator of

convolution, 33

degenerate kernel, 35

finite rank, 35

Hartley, 60

Hille-Tamarkin, 12, 57, 59, 64

Laplace, 63, 73

separable kernel, 35

Volterra, 60

Kwapień’s Theorem, 18

Laplace kernel, 64

Laplace transform, 63 
Lebesgue's Dominated Convergence

Theorem, 65

Lorentz space, 22

$L^{p}$-space, 15, 58, 63

$L^{q}$-improving measure, 33

Maurey-Rosenthal's Theorem, 6, 30

measurable set, 1

measurable space, 1

measure space, 1

Minkowski's integral inequality, 12

multiplier operator, 5

$\mu$-null set, 1

$m$-null set, 1,2

negative cone, 2

negative part, 2

Nikishin's Theorem, 10, 22

non-atomic measure, 6, 20

norming subspace, 66

operator, 3

order continuous

operator, 3

space, 3

Orlicz function, 17

Orlicz space, 17, 61

$(p, q)$-th power factorable operator, 37 , $38,40,45,54,58,63$

$p$-th power factorable adjoint operator,

25

$p$-th power factorable kernel operator,

35

$p$-th power factorable operator, $8,9,16$,

23,52

$p$-th power space, 6

perfect space, 3

positive cone, 2

positive part, 2

positive scalar measure, 1

probability measure, 43

quasi-Banach function space, 3 quasi-norm, 3

Rademacher function, 4

Radon-Nikodým derivative, 26, 27, 31, 34

Radon-Nikodým's Theorem, 43

regular pair, 47

Riesz transform, 17

$\delta$-ring, 44

Rybakov, 32

measure, 1, 30, 34, 69

$\sigma$-algebra, 1

generated, 2

Schauder's Theorem, 73

semivariation, 1

simple function, 2

space of multipliers, 5

type, 4,20

variation

of a scalar measure, 1

of a vector measure, 1

vector measure, 1

Vitali's Convergence Theorem, 66

Volterra measure, 33

Volterra operator, 33

weak order unit, 3

Young inequality, 8 